\title{
Exploration of the Solid-State Sorption Properties of Shape-persistent Macrocyclic Nanocarbons as Bulk Materials and Small Aggregates
}

Dr. Tobias A. Schaub, ${ }^{\star a, b, c}$ Ephraim Prantl, ${ }^{d}$ Julia Kohn, ${ }^{e}$ Markus Bursch, ${ }^{e}$ Checkers R. Marshall, ${ }^{a, b}$ Erik J. Leonhardt, ${ }^{a, b}$ Terri C. Lovell, ${ }^{a, b}$ Dr. Lev N. Zakharov, ${ }^{a}$ Prof. Carl K. Brozek, ${ }^{a}$ Prof. Siegfried R. Waldvogel, ${ }^{d}$ Prof. Stefan Grimme, ${ }^{e}$ Prof. Ramesh Jasti ${ }^{\star a, b}$

a Department of Chemistry \& Biochemistry and Material Science Institute, University of Oregon, Eugene, Oregon 97403, United States

${ }^{b}$ Knight Campus for Accelerating Scientific Impact, University of Oregon, Eugene, Oregon 97403, United States

c Institute of Organic Chemistry, Ruprecht-Karls University of Heidelberg, Heidelberg 69120, Germany

d Department of Organic Chemistry, Johannes Gutenberg-University Mainz, 55128, Germany

e Mulliken Center for Theoretical Chemistry, University Bonn, 53115, Germany

\section{Inhalt}

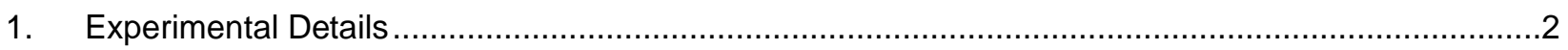

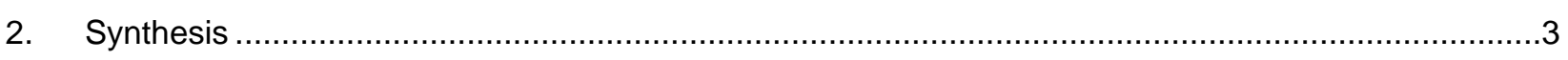

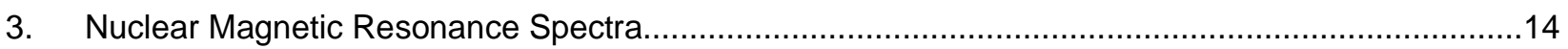

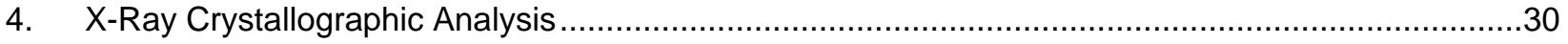

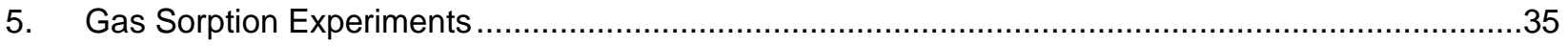

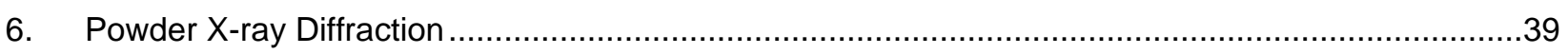

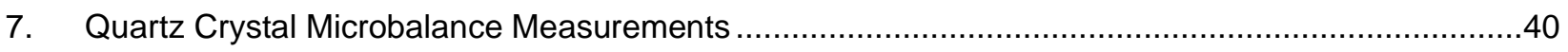

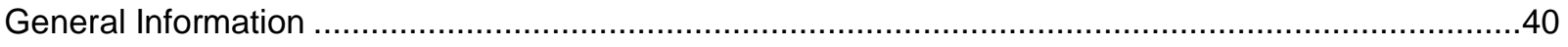

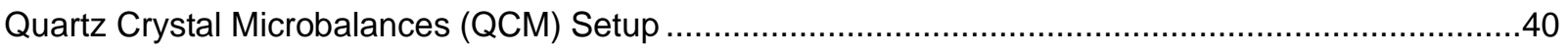

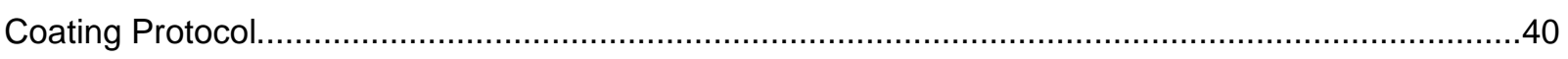

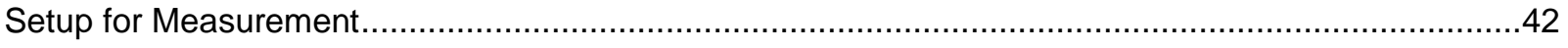

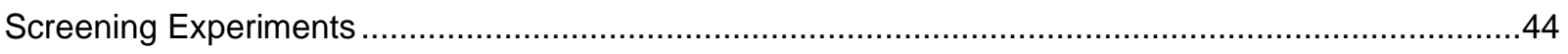

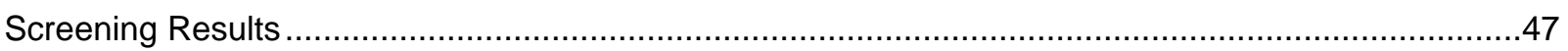

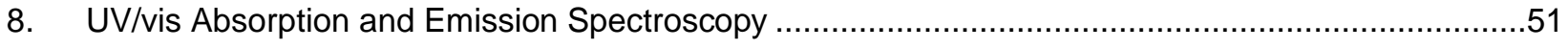

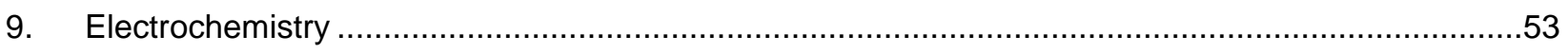

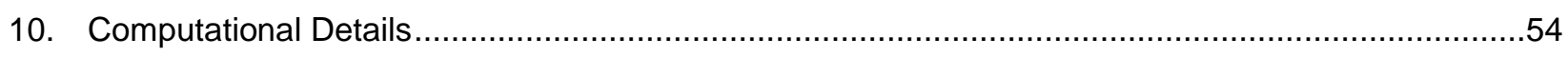

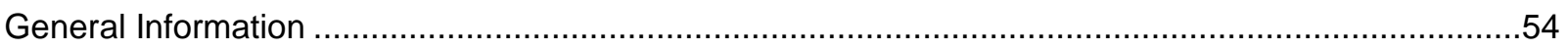

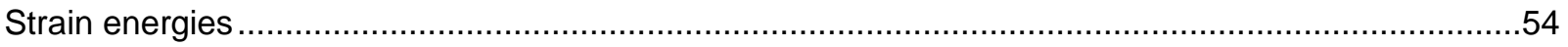

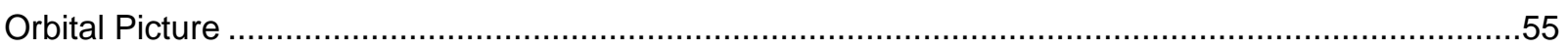

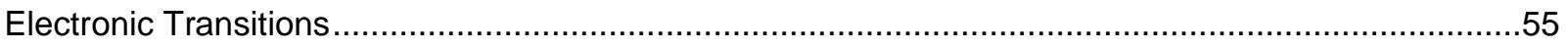

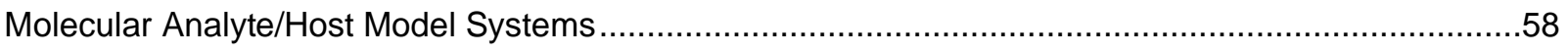

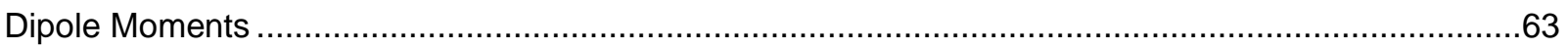

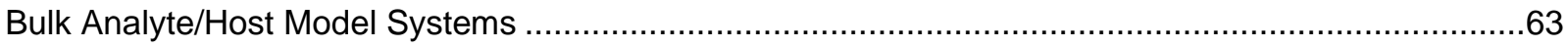

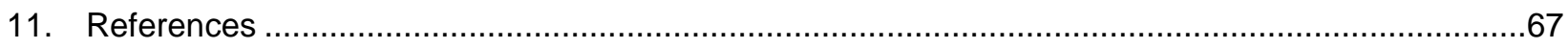




\section{Experimental Details}

General. Unless otherwise noted, commercially available materials were used without purification. $\mathbf{S} \mathbf{1}^{[1]}$ and $\mathbf{S} \mathbf{4}^{[2]}$ were prepared according to modified protocols from the literature. Moisture and oxygen sensitive reactions were carried out in flame-dried glassware and under an inert atmosphere of purified nitrogen using syringe/septa technique. Tetrahydrofuran (THF), and 1,4dioxane were dried by filtration through alumina according to the methods described by Grubbs. ${ }^{[3]}$ Thin-layer chromatography (TLC) was performed on the aluminium plates coated with $0.20 \mathrm{~mm}$ thickness of Silica Gel $60 \mathrm{~F}_{254}$ (Macherey-Nagel). Developing plates were visualized using UV light at wavelength of 254 and $365 \mathrm{~nm}$. Silica column chromatography was conducted with Zeochem Zeoprep n60 Eco 40-63 $\mu$ m silica gel.

${ }^{1} \mathrm{H}$ and ${ }^{13} \mathrm{C}$ NMR spectra were recorded on either a Bruker Avance III HD $500\left({ }^{1} \mathrm{H}: 500 \mathrm{MHz},{ }^{13} \mathrm{C}\right.$ : $126 \mathrm{MHz})$ or Bruker Avance III HD $600 \mathrm{MHz}\left({ }^{1} \mathrm{H}: 600 \mathrm{MHz},{ }^{13} \mathrm{C}: 151 \mathrm{MHz}\right) \mathrm{NMR}$ spectrometer equipped with a Prodigy multinuclear cryoprobe, respectively. The samples were measured at 25 ${ }^{\circ} \mathrm{C}$ if not stated otherwise. The chemical shifts $(\delta)$ were reported in parts per million (ppm) for and were referenced $\delta=7.26 \mathrm{ppm}\left({ }^{1} \mathrm{H}\right)$ and $\delta=77.2\left({ }^{13} \mathrm{C}\right)$ for $\mathrm{CDCl}_{3}$. Coupling constants $(\mathcal{J})$ are given in $\mathrm{Hz}$ and the apparent resonance multiplicity is reported as $\mathrm{s}$ (singlet), $\mathrm{d}$ (doublet), $\mathrm{t}$ (triplet), $\mathrm{q}$ (quartet), quint (quintet) or m (multiplet).

Infrared absorption (IR) spectra were recorded on a Thermo Scientific Nicolet 6700 spectrometer equipped with a diamond crystal Smart ATR. Characteristic IR absorptions are reported in $\mathrm{cm}^{-1}$ and denoted as strong (s), medium (m), and weak (w).

Cyclic voltammetry (CV) experiments were performed under $\mathrm{N}_{2}$ atmosphere using a Biologic SP50 Potentiostat and EC-labs software. A three-electrode setup was employed with a glassy carbon working electrode, a platinum coil counter electrode and a silver wire as pseudo reference electrode. The samples were measured in $0.1 \mathrm{M}$ electrolyte solutions of $\mathrm{NBu}_{4} \mathrm{PF}_{6}$ (used without further purification) in anhydrous THF and were purged with $\mathrm{N}_{2}$ for 20 min prior to analysis. The reported half-wave potentials are referenced to the ferrocene/ferrocenium $\left(\mathrm{Fc} / \mathrm{Fc}^{+}\right)$redox couple by adding ferrocene to the sample solution.

UV/vis absorption and fluorescence spectra were recorded on an Agilant Cary 100 spectrophotometer and a Horiba Jobin Yvon Fluoromax-4 Fluorometer, respectively. All measurements were carried out under ambient conditions in QS Quartz Suprasil cells (10 mm light path). The absorption maxima $\left(\lambda_{\max }\right)$ are reported in $\mathrm{nm}$ and the extinction coefficient $(\varepsilon)$ in $\mathrm{M}^{-1} \cdot \mathrm{Cm}^{-1}$. 


\section{Synthesis}
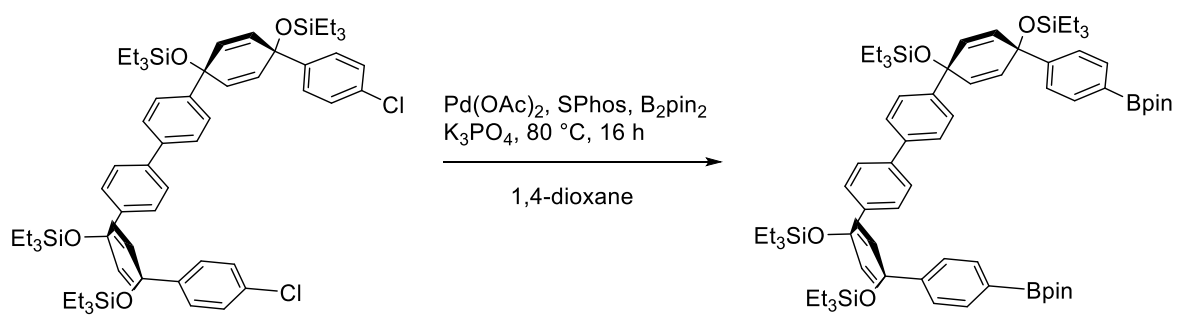

Compound 5. Starting S1 (3.00 g, 2.85 mmol, 1.00 equiv.), bis(pinacolato)diboron (4.34 g, 17.1 mmol, 6.00 equiv.), potassium acetate $(1.68 \mathrm{~g}, 17.1 \mathrm{mmol}, 6.00$ equiv.), palladium(II) acetate (32.0 $\mathrm{mg}, 0.14 \mathrm{mmol}, 5.00 \mathrm{~mol} \%$ ), and SPhos (146 mg, $0.36 \mathrm{mmol}, 13.0 \mathrm{~mol} \%$ ) were added to a flame dried flask and set under $\mathrm{N}_{2}$ atmosphere (potassium acetate was finely ground with a mortar and pestle and oven or flame dried immediately before use for best results). 1,4-Dioxane (anhydrous, $\mathrm{N}_{2}$ sparged for $30 \mathrm{~min}, 95 \mathrm{~mL}, 0.03 \mathrm{M}$ ) was added and the resulting solution was sparged for $1 \mathrm{~h}$ with $\mathrm{N}_{2}$. The reaction was heated to $80^{\circ} \mathrm{C}$ for $12 \mathrm{~h}$ at which point it was cooled to room temperature and filtered over a plug of Celite. The crude mixture was concentrated to afford a colorless viscous oil, which was triturated with cold ethanol to give product 5 as a white solid $(3.19 \mathrm{~g}, 2.58 \mathrm{mmol}$, $91 \%) . R_{\mathrm{f}}=0.45\left(\mathrm{SiO}_{2}, 50 \% \mathrm{CH}_{2} \mathrm{Cl}_{2} /\right.$ hexanes $)$, the product is decomposing on silica; $\mathrm{Mp} 182.1-$ $183.1{ }^{\circ} \mathrm{C} ;{ }^{1} \mathrm{H}$ NMR $\left(600 \mathrm{MHz}, \mathrm{CDCl}_{3}\right) \delta 7.73(\mathrm{~d}, J=8.3 \mathrm{~Hz}, 4 \mathrm{H}), 7.49$ (d, $\left.J=8.3 \mathrm{~Hz}, 4 \mathrm{H}\right), 7.39$ (dd, $J=8.3,1.7 \mathrm{~Hz}, 8 \mathrm{H}), 6.00(\mathrm{~d}, J=2.3 \mathrm{~Hz}, 8 \mathrm{H}), 1.33(\mathrm{~s}, 24 \mathrm{H}), 0.95(\mathrm{t}, J=8.0 \mathrm{~Hz}, 18 \mathrm{H}), 0.93(\mathrm{t}, J=$ $8.0 \mathrm{~Hz}, 18 \mathrm{H}$ ), 0.64 (q, $J=8.0 \mathrm{~Hz}, 12 \mathrm{H}), 0.60$ (q, $J=8.0 \mathrm{~Hz}, 12 \mathrm{H}) \mathrm{ppm} ;{ }^{13} \mathrm{C} \mathrm{NMR}\left(151 \mathrm{MHz}, \mathrm{CDCl}_{3}\right)$ $\delta$ 149.4, 145.2, 139.8, 134.9, 131.8, 131.5, 127.0, 126.4, 125.4, 83.9, 71.7, 71.5, 25.1, 7.27, 7.25, 6.6 ppm; two signals coincide; IR (ATR): $\tilde{v} 2953$ (w), 2989 (w), 2875 (w), 1609 (w), 1360 (s), 1143 (w), 1061 (s), 1003 (m), 955 (s), 859 (m), 731 (s) cm ${ }^{-1}$; HRMS (TOF, ESI, positive mode) m/z calcd for $\mathrm{C}_{72} \mathrm{H}_{108}{ }^{11} \mathrm{~B}_{2} \mathrm{NaO}_{8} \mathrm{Si}_{4}: 1257.7200[\mathrm{M}+\mathrm{Na}]^{+}$, found 1257.7258.

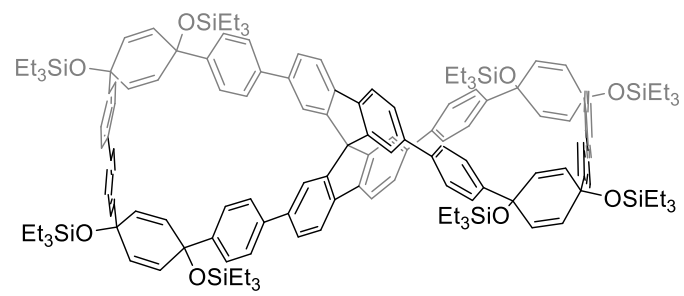

Compound 9. Compound S1 (1.86 g, 1.50 mmol, 2.20 equiv.), 2,2',7,7'-tetrabromo-9,9'spirobifluorene (0.43 g, $0.68 \mathrm{mmol}, 1.00$ equiv.), and (2-dicyclohexylphosphino-2',6'dimethoxybiphenyl)[2-(2'-amino-1,1'-biphenyl)]palladium(II) methanesulfonate (SPhos Pd G3, $26.6 \mathrm{mg}, 0.03 \mathrm{mmol}, 5.0 \mathrm{~mol} \%$ ) were added to 1,4-dioxane (anhydrous, $\mathrm{N}_{2}$ sparged for $30 \mathrm{~min}, 68$ $\mathrm{mL}, 0.01 \mathrm{M})$ and the resulting solution was sparged with $\mathrm{N}_{2}$ for $1 \mathrm{~h}$. The reaction mixture was heated to $80{ }^{\circ} \mathrm{C}$ followed by addition of aqueous $\mathrm{K}_{3} \mathrm{PO}_{4}$ solution $\left(\mathrm{N}_{2}\right.$ sparged for $1 \mathrm{~h}, 2.0 \mathrm{M}, 7.0$ $\mathrm{mL}$, exc.) via syringe. The reaction was stirred at $80^{\circ} \mathrm{C}$ for $1 \mathrm{~h}$ before it was allowed to cool to room temperature and water was added $(200 \mathrm{~mL})$. The product was extracted with dichloromethane $(3 \times 100 \mathrm{~mL})$. The combined organic fractions were washed with water $(1 \times 100$ $\mathrm{mL})$, and brine $(1 \times 100 \mathrm{~mL})$, dried over sodium sulfate and the solvent was removed. The resulting 
brownish crude product was purified by column chromatography $\left(\mathrm{SiO}_{2}, 0-10 \% \mathrm{EtOAc/hexanes)}\right.$ to yield product 9 together with small amounts of polymeric material as a white solid $(0.85 \mathrm{~g}, 0.37$ $\mathrm{mmol}, 55 \%)$. Analytically pure samples can be obtained by gel permeation chromatography $\left(\mathrm{CHCl}_{3}\right) \cdot R_{\mathrm{f}}=0.58\left(\mathrm{SiO}_{2}, 50 \% \mathrm{CH}_{2} \mathrm{Cl}_{2} /\right.$ hexanes $) ; \mathrm{Mp}>300{ }^{\circ} \mathrm{C} ;{ }^{1} \mathrm{H} \mathrm{NMR}\left(600 \mathrm{MHz}, \mathrm{CDCl}_{3}\right) \delta 7.92$ (d, $J=7.9 \mathrm{~Hz}, 4 \mathrm{H}), 7.60$ (dd, $J=7.9,1.6 \mathrm{~Hz}, 4 \mathrm{H}), 7.34(\mathrm{~s}, 16 \mathrm{H}), 7.20(\mathrm{~d}, J=8.5 \mathrm{~Hz}, 8 \mathrm{H}), 7.14$ (d, $J=8.5 \mathrm{~Hz}, 8 \mathrm{H}), 7.01(\mathrm{~d}, J=1.6 \mathrm{~Hz}, 4 \mathrm{H}), 6.14(\mathrm{dd}, J=10.1,2.4 \mathrm{~Hz}, 4 \mathrm{H}), 6.05(\mathrm{dd}, J=10.1,2.4$ $\mathrm{Hz}, 4 \mathrm{H}), 5.96$ (dd, $J=10.1,2.4 \mathrm{~Hz}, 4 \mathrm{H}), 5.91$ (dd, $J=10.1,2.4 \mathrm{~Hz}, 4 \mathrm{H}), 0.98$ (t, $J=7.9 \mathrm{~Hz}, 36 \mathrm{H}$ ), $0.88(\mathrm{t}, J=7.9 \mathrm{~Hz}, 36 \mathrm{H}), 0.68(\mathrm{q}, J=7.9 \mathrm{~Hz}, 24 \mathrm{H}), 0.53(\mathrm{q}, J=7.9 \mathrm{~Hz}, 24 \mathrm{H}) \mathrm{ppm} ;{ }^{13} \mathrm{C}$ NMR $(151$ $\left.\mathrm{MHz}_{2} \mathrm{CDCl}_{3}\right) \delta 149.8,144.8,144.3,141.3,140.8,140.6$ 139.7, 133.1, 132.0, 131.8, 130.5, 127.6, 127.2, 126.8, 126.7, 126.2, 123.1, 120.4, 72.2, 71.3, 7.3, 7.2, 6.61, 6.59 ppm, one signals coincide; IR (ATR): ṽ 3030 (w), 2950 (m), 2909 (w), $2873(\mathrm{~m}), 1489$ (w), $1463(\mathrm{~m}), 1398(\mathrm{~m}), 1238(\mathrm{~m})$, 1178 (m), 1071 (s), 1004 (s), 960 (s), 859 (s), 814 (s) cm'-1 MALDI-MS (DCTB, positive mode) $\mathrm{m} / \mathrm{z}$ calcd for $\mathrm{C}_{145} \mathrm{H}_{181} \mathrm{O}_{8} \mathrm{Si}_{8}: 2274.190[\mathrm{M}+\mathrm{H}]^{+}$; found 2274.166 .

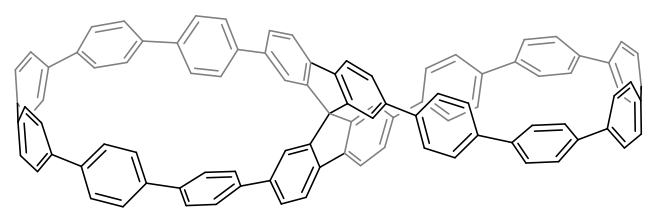

Compound 1. To a solution of compound 9 (1.10 g, $0.48 \mathrm{mmol}, 1.00$ equiv.) in THF (24 $\mathrm{mL}, 0.02$ M) tetrabutylammonium fluoride (TBAF, $1.0 \mathrm{M}, 5.8 \mathrm{~mL}, 5.8 \mathrm{mmol}, 12.0$ equiv.) was added at room temperature and the reaction mixture stirred for $4 \mathrm{~h}$. Water $(50 \mathrm{~mL})$ was added and the THF was almost completely evaporated under reduced pressure. The resulting solid was finely dispersed in an ultrasonication bath and filtered off and thoroughly washed with water and $\mathrm{CH}_{2} \mathrm{Cl}_{2}$ to afford the deprotected macrocycle as an off-white solid $(0.62 \mathrm{~g}, 0.46 \mathrm{mmol}, 94 \%)$. In the second step, to a solution of $\mathrm{SnCl}_{2} \cdot 2 \mathrm{H}_{2} \mathrm{O}(0.62 \mathrm{~g}, 2.73 \mathrm{mmol}, 6.00$ equiv.) in THF (14 mL, $0.02 \mathrm{M})$ was added concentrated aqueous $\mathrm{HCl}(0.44 \mathrm{~mL}, 5.32 \mathrm{mmol}, 11.7$ equiv.) at room temperature and the resulting solution was stirred for $15 \mathrm{~min}$ under nitrogen atmosphere. The resulting mixture was added to a dispersion of the as prepared deprotected macrocycle $(0.62 \mathrm{~g}, 0.46 \mathrm{mmol}, 1.00$ equiv.) in THF (46 mL, $0.01 \mathrm{M}$ ) and the mixture was stirred for 50 min at room temperature. The reaction mixture was concentrated under reduced pressure (ca. $20 \mathrm{~mL}$ ) and directly subjected to column chromatography (basic alumina, $50 \% \mathrm{CH}_{2} \mathrm{Cl}_{2} /$ hexanes) to yield the product $\mathbf{1}$ as a yellow, bluegreen fluorescent solid $(0.26 \mathrm{~g}, 0.21 \mathrm{mmol}, 47 \%)$. The product can be stored under ambient conditions for months without noticeable decomposition. $R_{\mathrm{f}}=0.27\left(\mathrm{SiO}_{2}, 50 \% \mathrm{CH}_{2} \mathrm{Cl}_{2} / \mathrm{hexanes}\right)$; $\mathrm{Mp}>300{ }^{\circ} \mathrm{C} ;{ }^{1} \mathrm{H}$ NMR $\left(600 \mathrm{MHz}\right.$, THF-d 8 ) $\delta{ }^{1} \mathrm{H}$ NMR $\left(600 \mathrm{MHz}, \mathrm{CDCl}_{3}\right) \delta 7.91(\mathrm{~d}, J=8.1 \mathrm{~Hz}, 4 \mathrm{H})$, $7.68(\mathrm{dd}, J=8.1,1.7 \mathrm{~Hz}, 4 \mathrm{H}), 7.62(\mathrm{br}, \mathrm{d}, J=9.2 \mathrm{~Hz}, 4 \mathrm{H}), 7.56(\mathrm{br}, \mathrm{d}, J=9.2 \mathrm{~Hz}, 4 \mathrm{H}), 7.51-7.41$ (br, m, 12H), $7.40(\mathrm{~d}, J=8.3 \mathrm{~Hz}, 8 \mathrm{H}), 7.36(\mathrm{~d}, J=8.3 \mathrm{~Hz}, 12 \mathrm{H}), 7.30(\mathrm{~d}, J=8.3 \mathrm{~Hz}, 8 \mathrm{H}), 7.02(\mathrm{~d}$, $J=1.7 \mathrm{~Hz}, 4 \mathrm{H}) \mathrm{ppm} ;{ }^{13} \mathrm{C}$ NMR $\left(151 \mathrm{MHz}, \mathrm{THF}-\mathrm{d}_{8}\right) \delta{ }^{13} \mathrm{C} N M R\left(151 \mathrm{MHz}, \mathrm{CDCl}_{3}\right) \delta 150.0,140.8$, 140.0, 139.2, 138.6, 138.4, 138.1, 137.9, 137.1, 129.3, 128.6, 127.8, 127.5, 127.0, 126.2, 121.6, 120.5, 67.21 ppm, signal coincides; IR (ATR): v 3023 (w), 2949 (w), 2921 (w), 2862 (w), 1584 (w) 1489 (w), 1463 (m), 1393 (w), 1244 (w), 1112 (w), 1000 (m), 808 (s) cm'-1 HRMS (TOF, ESI, positive mode) $\mathrm{m} / z$ calcd for $\mathrm{C}_{97} \mathrm{H}_{60}: 1224.4690[\mathrm{M}]^{+}$, found 1224.4660 . 


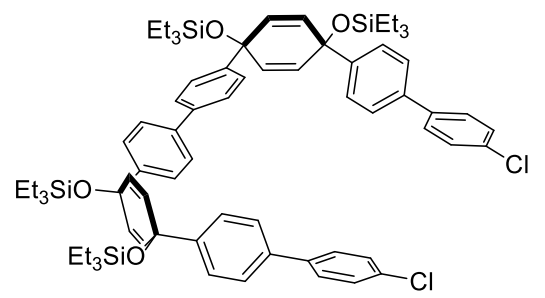

Compound S2. Compound 5 (7.00 g, 5.67 mmol, 1.00 equiv.), 1-bromo-4-chlorobenzene (2.39 g, $12.5 \mathrm{mmol}, 2.20$ equiv.), and [1,1-bis(diphenylphosphino)ferrocene]dichloropalladium(II) $\left(\mathrm{Pd}(\mathrm{dppf}) \mathrm{Cl}_{2}, 138.8 \mathrm{mg}, 170.0 \mu \mathrm{mol}, 3.0 \mathrm{~mol} \%\right.$ ) were added to 1,4-dioxane (anhydrous, $\mathrm{N}_{2}$ sparged for $30 \mathrm{~min}, 113 \mathrm{~mL}, 0.05 \mathrm{M}$ ) and the resulting solution was sparged with $\mathrm{N}_{2}$ for $1 \mathrm{~h}$. The reaction mixture was heated to $70^{\circ} \mathrm{C}$ followed by addition of aqueous $\mathrm{K}_{3} \mathrm{PO}_{4}$ solution $\left(\mathrm{N}_{2}\right.$ sparged for $1 \mathrm{~h}, 2.0 \mathrm{M}, 14.2 \mathrm{~mL}$, exc.) via syringe. The reaction was stirred at $70{ }^{\circ} \mathrm{C}$ for $16 \mathrm{~h}$ before it was allowed to cool to room temperature and water was added $(100 \mathrm{~mL})$. The product was extracted with EtOAc $(3 \times 100 \mathrm{~mL})$. The combined organic fractions were washed with water $(1 \times 50 \mathrm{~mL})$, and brine $(1 \times 50 \mathrm{~mL})$, dried over sodium sulfate. The mixture was filtered through a plug of Celite and the solvent was removed to give a colourless oily residue. $\mathrm{EtOH}(30 \mathrm{~mL})$ was added and the formed precipitate filtered off to yield the product $\mathbf{S 2}$ as a white solid $(6.40 \mathrm{~g}, 5.31 \mathrm{mmol}, 94 \%) . R_{\mathrm{f}}$ $=0.73\left(\mathrm{SiO}_{2}, 50 \% \mathrm{CH}_{2} \mathrm{Cl}_{2} /\right.$ hexanes$) ; \mathrm{Mp} 160.8-161.4^{\circ} \mathrm{C} ;{ }^{1} \mathrm{H} \mathrm{NMR}\left(500 \mathrm{MHz}, \mathrm{CDCl}_{3}\right) \delta 7.51$ (dd, $J=8.5,5.8 \mathrm{~Hz}, 8 \mathrm{H}), 7.49-7.45(\mathrm{~m}, 4 \mathrm{H}), 7.43(\mathrm{~d}, J=3.3 \mathrm{~Hz}, 4 \mathrm{H}), 7.42(\mathrm{~d}, J=3.3 \mathrm{~Hz}, 4 \mathrm{H}), 7.38$ $(\mathrm{d}, J=8.5 \mathrm{~Hz}, 4 \mathrm{H}), 6.07-6.02(\mathrm{~m}, 8 \mathrm{H}), 0.96(\mathrm{td}, J=8.0,4.6 \mathrm{~Hz}, 36 \mathrm{H}), 0.64(\mathrm{dq}, J=8.0,7.7 \mathrm{~Hz}$, $24 \mathrm{H}) \mathrm{ppm} ;{ }^{13} \mathrm{C}$ NMR $\left(126 \mathrm{MHz}, \mathrm{CDCl}_{3}\right) \delta 145.7,145.3,139.8,139.4,138.9,133.5,131.8,131.6$, 129.1, 128.5, 126.93, 126.86, 126.6, 126.5, 71.6, 71.5, 7.3, 6.7 ppm, two signals coincide; IR (ATR): $\tilde{v} 3030$ (w), 2952 (m), 2909 (w), 2874 (m), $1484(\mathrm{~m}), 1456$ (w), 1412 (w), 1399 (w), 1238 (m), 1181 (w), 1068 (s), 1004 (s), 959 (s), 861 (m), 812 (s) cm ${ }^{-1}$; HRMS (ESI-TOF, positive mode) $\mathrm{m} / \mathrm{z}$ calcd for $\mathrm{C}_{72} \mathrm{H}_{92}{ }^{35} \mathrm{Cl}_{2} \mathrm{O}_{4} \mathrm{Si}_{4}: 1202.5449[\mathrm{M}]^{+}$, found 1202.5269 .

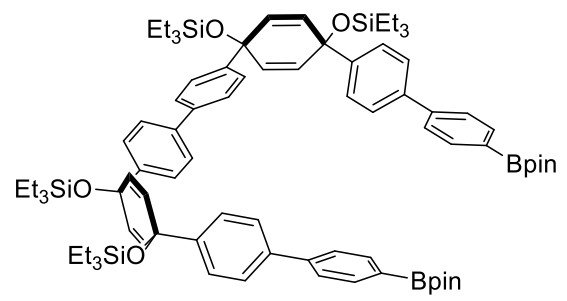

Compound 6. Starting S2 (6.00 g, $4.98 \mathrm{mmol}, 1.00$ equiv), bis(pinacolato)diboron (7.59 g, 29.9 mmol, 6.00 equiv.), potassium acetate $(2.93 \mathrm{~g}, 29.9 \mathrm{mmol}, 6.00$ equiv.), palladium(II) acetate (33.5 $\mathrm{mg}, 0.15 \mathrm{mmol}, 3.0 \mathrm{~mol} \%$ ) and SPhos (122.7 mg, $0.30 \mathrm{mmol}, 6.0 \mathrm{~mol} \%)$ were added to a flame dried flask and set under $\mathrm{N}_{2}$ atmosphere (potassium acetate was finely ground with a mortar and pestle and oven or flame dried immediately before use for best results). 1,4-Dioxane (anhydrous, $\mathrm{N}_{2}$ sparged for $30 \mathrm{~min}, 100 \mathrm{~mL}, 0.05 \mathrm{M}$ ) was added and the resulting solution was sparged for 1 $\mathrm{h}$ with $\mathrm{N}_{2}$. The reaction was heated to $80^{\circ} \mathrm{C}$ for $12 \mathrm{~h}$ at which point it was cooled to room temperature and filtered over a plug of Celite. The crude mixture was concentrated to afford a colorless viscous oil, which was triturated with cold ethanol to give product 6 as a white solid (5.84 $\mathrm{g}, 4.21 \mathrm{mmol}, 84 \%) . R_{\mathrm{f}}=0.38\left(\mathrm{SiO}_{2}, 50 \% \mathrm{CH}_{2} \mathrm{Cl}_{2} /\right.$ hexanes$)$, the product is decomposing on silica; Mp $127.5-132.2^{\circ} \mathrm{C} ;{ }^{1} \mathrm{H}$ NMR $\left(600 \mathrm{MHz}, \mathrm{CDCl}_{3}\right) \delta 7.86(\mathrm{~d}, J=8.0 \mathrm{~Hz}, 4 \mathrm{H}), 7.59(\mathrm{~d}, J=8.0 \mathrm{~Hz}$, $4 \mathrm{H}), 7.53(\mathrm{dd}, J=7.9,7.9 \mathrm{~Hz}, 8 \mathrm{H}), 7.43(\mathrm{dd}, J=8.3,2.7 \mathrm{~Hz}, 8 \mathrm{H}), 6.04(\mathrm{~d}, J=2.7 \mathrm{~Hz}, 8 \mathrm{H}), 1.36$ 
(s, 24H), 0.96 (td, $J=8.0,5.8 \mathrm{~Hz}, 36 \mathrm{H}), 0.64(\mathrm{dq}, J=8.0,8.1 \mathrm{~Hz}, 24 \mathrm{H}) \mathrm{ppm} ;{ }^{13} \mathrm{C}$ NMR $(151 \mathrm{MHz}$, $\left.\mathrm{CDCl}_{3}\right) \delta 145.7,145.3,143.6,140.0,139.8,135.4,131.72,131.68,127.1,127.0,126.53,126.50$, 84.0, 71.6, 71.5, 25.1 , 7.3, 6.7 pm; four signals coincide; $3029(\mathrm{w}), 2952(\mathrm{~m}), 2909(\mathrm{w}), 2874(\mathrm{~m})$, 1609 (w), 1457 (w), 1397 (w), 1358 (s), 1321 (w), 1237 (w), 1143 (m), 1070 (s), 1004 (s), 960 (s), $860(\mathrm{~m}), 818(\mathrm{~s}) \mathrm{cm}^{-1}$; HRMS (ESI-TOF, positive mode) $\mathrm{m} / z$ calcd for $\mathrm{C}_{84} \mathrm{H}_{116}{ }^{11} \mathrm{~B}_{2} \mathrm{O}_{8} \mathrm{Si}_{4}: 1386.7933$ $[\mathrm{M}]^{+}$, found 1386.7778 .

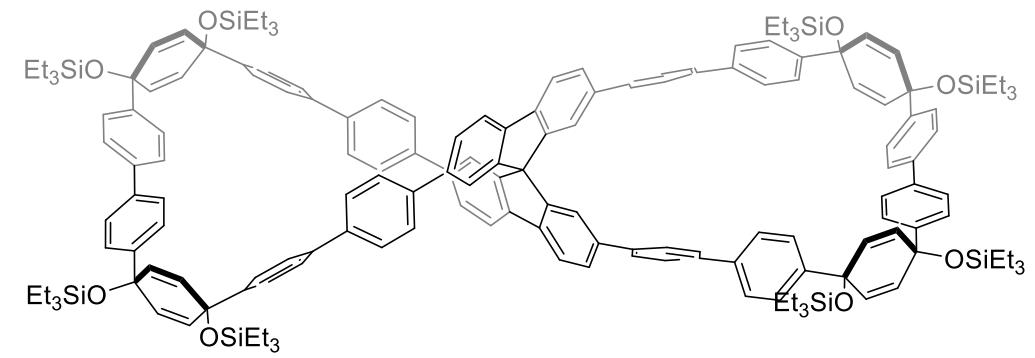

Compound 10. 2,2',7,7'-Tetrabromo-9,9'-spirobifluorene (0.50 g, $0.79 \mathrm{mmol}, 1.00$ equiv.), compound 6 (2.42 g, 1.74 mmol, 2.20 equiv.), and (2-dicyclohexylphosphino-2',6'dimethoxybiphenyl)[2-(2'-amino-1,1'-biphenyl)]palladium(II)methanesulfonate (SPhos Pd G3, $30.9 \mathrm{mg}, 39.6 \mu \mathrm{mol}, 5.0 \mathrm{~mol} \%$ ) were added to 1,4-dioxane (anhydrous, $\mathrm{N}_{2}$ sparged for $30 \mathrm{~min}$, $158 \mathrm{~mL}, 0.005 \mathrm{M}$ ) and the resulting solution was sparged with $\mathrm{N}_{2}$ for $1 \mathrm{~h}$. The reaction mixture was heated to $80{ }^{\circ} \mathrm{C}$ followed by addition of aqueous $\mathrm{K}_{3} \mathrm{PO}_{4}$ solution $\left(\mathrm{N}_{2}\right.$ sparged for $1 \mathrm{~h}, 2.0 \mathrm{M}, 15.8$ $\mathrm{mL}$, exc.) via syringe. The reaction was stirred at $80^{\circ} \mathrm{C}$ for $16 \mathrm{~h}$ before it was allowed to cool to room temperature and water was added $(200 \mathrm{~mL})$. The product was extracted with dichloromethane $(3 \times 100 \mathrm{~mL})$. The combined organic fractions were washed with water $(1 \times 100$ $\mathrm{mL})$, and brine $(1 \times 100 \mathrm{~mL})$, dried over sodium sulfate and the solvent was removed. The resulting brownish crude product was purified by column chromatography $\left(\mathrm{SiO}_{2}, 0-10 \% \mathrm{EtOAc/hexanes}\right)$ to yield product 10 together with small amounts of polymeric material as a white solid $(0.61 \mathrm{~g}, 0.24$ $\mathrm{mmol}, 30 \%)$. Analytically pure samples can be obtained by gel permeation chromatography $\left(\mathrm{CHCl}_{3}\right) \cdot R_{\mathrm{f}}=0.51\left(\mathrm{SiO}_{2}, 50 \% \mathrm{CH}_{2} \mathrm{Cl}_{2} /\right.$ hexanes $) ; \mathrm{Mp}>300{ }^{\circ} \mathrm{C} ;{ }^{1} \mathrm{H} \mathrm{NMR}\left(500 \mathrm{MHz}, \mathrm{CDCl}_{3}\right) \delta 7.99$ $(\mathrm{d}, J=8.0 \mathrm{~Hz}, 4 \mathrm{H}), 7.74(\mathrm{dd}, J=8.0,1.7 \mathrm{~Hz}, 4 \mathrm{H}), 7.57-7.47(\mathrm{~m}, 32 \mathrm{H}), 7.44(\mathrm{dd}, J=8.1,8.1 \mathrm{~Hz}$, $17 \mathrm{H}), 7.06(\mathrm{~d}, J=1.7 \mathrm{~Hz}, 4 \mathrm{H}), 6.07-5.97(\mathrm{~m}, 16 \mathrm{H}), 0.97(\mathrm{dt}, J=8.0,8.0 \mathrm{~Hz}, 72 \mathrm{H}), 0.65(\mathrm{dq}, J=$ 15.8, $8.0 \mathrm{~Hz}, 48 \mathrm{H}) \mathrm{ppm} ;{ }^{13} \mathrm{C}$ NMR $\left(126 \mathrm{MHz}, \mathrm{CDCl}_{3}\right) \delta 150.4,145.5,145.4,141.1,140.7,139.8$, 139.73, 139.71, 139.6, 132.7, 132.1, 131.7, 131.1, 130.6, 127.5, 127.4, 127.0, 126.92, 126.88, 126.5, 126.3, 123.5, 120.7, 71.6, 71.4, 66.8, 7.3, 6.67, 6.65 ppm; IR (ATR): $\tilde{v} 3028$ (w), 2951 (m), 2908 (w), $2873(\mathrm{~m}), 1490(\mathrm{w}), 1462(\mathrm{~m}), 1398(\mathrm{w}), 1238(\mathrm{~m}), 1177(\mathrm{~m}), 1066(\mathrm{~s}), 1003(\mathrm{~s}), 958$ (s), $862(\mathrm{~m}), 811$ (s) $\mathrm{cm}^{-1}$; MALDI-MS (DCTB, positive mode) $\mathrm{m} / z$ calcd for $\mathrm{C}_{169} \mathrm{H}_{196} \mathrm{O}_{8} \mathrm{Si}_{8}$ : 2577.3084 [M] $]^{+}$; found 2577.3170. 


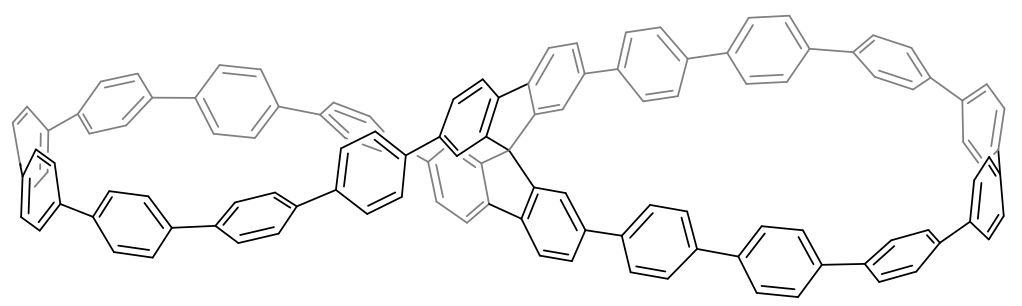

Compound 2. To a solution of compound $10(0.61 \mathrm{~g}, 0.24 \mathrm{mmol}, 1.00$ equiv.) in THF (12 mL, 0.02 M) tetrabutylammonium fluoride (TBAF, $1.0 \mathrm{M}, 2.8 \mathrm{~mL}, 2.8 \mathrm{mmol}, 12.0$ equiv.) was added at room temperature and the reaction mixture stirred for $4 \mathrm{~h}$. Water $(20 \mathrm{~mL})$ was added and the THF was almost completely evaporated under reduced pressure. The resulting solid was finely dispersed in an ultrasonication bath, filtered off, and thoroughly washed with water and $\mathrm{CH}_{2} \mathrm{Cl}_{2}$ to afford the deprotected macrocycle as an off-white solid $(0.377 \mathrm{~g}, 0.23 \mathrm{mmol}, 96 \%)$. In the next step, to a solution of $\mathrm{SnCl}_{2} \cdot 2 \mathrm{H}_{2} \mathrm{O}(0.29 \mathrm{~g}, 1.26 \mathrm{mmol}, 6.00$ equiv. $)$ in THF $(6.0 \mathrm{~mL}, 0.2 \mathrm{M})$ was added concentrated aqueous $\mathrm{HCl}(0.21 \mathrm{~mL}, 2.46 \mathrm{mmol}, 11.7$ equiv. $)$ at room temperature and the resulting solution was stirred for 15 min under nitrogen atmosphere. The resulting mixture was added to a dispersion of the as prepared deprotected macrocycle $(0.35 \mathrm{~g}, 0.21 \mathrm{mmol}, 1.00$ equiv.) in THF ( $21 \mathrm{~mL}, 0.01 \mathrm{M})$ and the mixture was stirred for $50 \mathrm{~min}$ at room temperature. The reaction mixture was concentrated under reduced pressure (ca. $10 \mathrm{~mL}$ ) and directly subjected to column chromatography (basic alumina, $50 \% \mathrm{CH}_{2} \mathrm{Cl}_{2}$ /hexanes) to yield product 2 as a yellowish, blue fluorescent solid $(0.13 \mathrm{~g}, 85.6 \mu \mathrm{mol}, 41 \%)$. The product can be stored under ambient conditions for months without noticeable decomposition. $R_{\mathrm{f}}=0.24\left(\mathrm{SiO}_{2}, 50 \% \mathrm{CH}_{2} \mathrm{Cl}_{2} /\right.$ hexanes $) ; \mathrm{Mp}>300$ ${ }^{\circ} \mathrm{C} ;{ }^{1} \mathrm{H}$ NMR $\left(500 \mathrm{MHz}, \mathrm{CDCl}_{3}\right) \delta 7.96(\mathrm{~d}, J=8.1 \mathrm{~Hz}, 4 \mathrm{H}), 7.72(\mathrm{dd}, J=8.1,1.7 \mathrm{~Hz}, 4 \mathrm{H}), 7.57-$ $7.42(\mathrm{~m}, 64 \mathrm{H}), 7.11(\mathrm{~d}, J=1.6 \mathrm{~Hz}, 4 \mathrm{H}) \mathrm{ppm} ;{ }^{13} \mathrm{C} \mathrm{NMR}\left(126 \mathrm{MHz}, \mathrm{CDCl}_{3}\right) \delta 150.0,140.9,140.1$, 139.4, 139.1, 138.82, 138.80, 138.6, 138.2, 138.0, 137.9, 137.8, 127.9, 127.74, 127.70, 127.6, 127.51, 127.47, 127.4, 127.3, 121.9, 120.6, 67.0 ppm; IR (ATR): $\tilde{v} 3024$ (w), 2952 (w), 2919 (w), 2868 (w), 1591 (w) $1485(\mathrm{~m}), 1462(\mathrm{~m}), 1387(\mathrm{w}), 1243$ (w), $1111(\mathrm{w}), 1001(\mathrm{~m}), 807$ (s) cm ${ }^{-1}$; HRMS (MALDI, DCTB, positive mode) $\mathrm{m} / z$ calcd for $\mathrm{C}_{121} \mathrm{H}_{76}: 1528.5947$ [M] $^{+}$, found 1528.5907 .

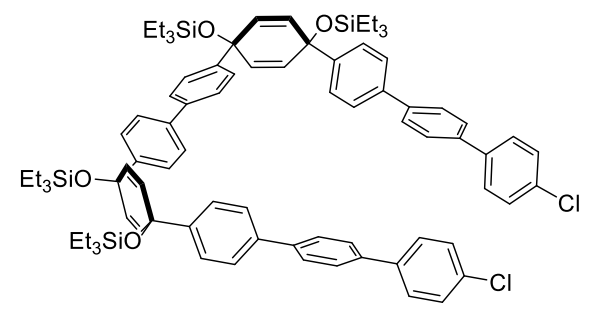

Compound S3. Compound 5 ( $1.80 \mathrm{~g}, 1.46 \mathrm{mmol}, 1.00$ equiv.), 4-chloro-4'-bromobiphenyl ( 0.86 $\mathrm{g}, 3.2 \mathrm{mmol}, 2.20$ equiv.), and [1,1-bis(diphenylphosphino)ferrocene]dichloropalladium(II) $\left(\mathrm{Pd}(\mathrm{dppf}) \mathrm{Cl}_{2}, 59.5 \mathrm{mg}, 72.8 \mu \mathrm{mol}, 5.0 \mathrm{~mol} \%\right.$ ) were added to 1,4-dioxane (anhydrous, $\mathrm{N}_{2}$ sparged for $30 \mathrm{~min}, 29 \mathrm{~mL}, 0.05 \mathrm{M}$ ) and the resulting solution was sparged with $\mathrm{N}_{2}$ for $1 \mathrm{~h}$. The reaction mixture was heated to $80^{\circ} \mathrm{C}$ followed by addition of aqueous $\mathrm{K}_{3} \mathrm{PO}_{4}$ solution $\left(\mathrm{N}_{2}\right.$ sparged for $1 \mathrm{~h}$, 2.0 M, $3.6 \mathrm{~mL}$, exc.) via syringe. The reaction was stirred at $70^{\circ} \mathrm{C}$ for $16 \mathrm{~h}$ before it was allowed to cool to room temperature and water was added $(50 \mathrm{~mL})$. The product was extracted with EtOAc $(3 \times 50 \mathrm{~mL})$. The combined organic fractions were washed with water $(1 \times 50 \mathrm{~mL})$, and brine $(1 \times$ $50 \mathrm{~mL}$ ), dried over sodium sulfate. The mixture was filtered through a plug of Celite and the solvent was removed to give a colourless oily residue. $\mathrm{EtOH}(20 \mathrm{~mL})$ was added and the formed precipitate 
filtered off to yield the product $\mathbf{S} 3$ as a white solid $(1.85 \mathrm{~g}, 1.37 \mathrm{mmol}, 94 \%) . R_{\mathrm{f}}=0.71\left(\mathrm{SiO}_{2}, 50 \%\right.$ $\mathrm{CH}_{2} \mathrm{Cl}_{2} /$ hexanes); Mp 106.5 - $107.9^{\circ} \mathrm{C}$ (decomp.); ${ }^{1} \mathrm{H}$ NMR $\left(500 \mathrm{MHz}, \mathrm{CDCl}_{3}\right) \delta 7.66(\mathrm{~d}, J=7.9$ $\mathrm{Hz}, 4 \mathrm{H}), 7.59$ (d, J = 7.9 Hz, 4H), $7.56-7.52(\mathrm{~m}, 12 \mathrm{H}), 7.44$ (dd, J = 7.7, $7.7 \mathrm{~Hz}, 8 \mathrm{H}), 7.40$ (d, J $=8.2 \mathrm{~Hz}, 4 \mathrm{H}), 6.06(\mathrm{~s}, 8 \mathrm{H}), 0.97(\mathrm{td}, J=8.1,7.4 \mathrm{~Hz}, 36 \mathrm{H}), 0.66(\mathrm{dq}, J=8.1,8.1 \mathrm{~Hz}, 24 \mathrm{H}) \mathrm{ppm}$; ${ }^{13} \mathrm{C}$ NMR $\left(126 \mathrm{MHz} \mathrm{CDCl}_{3}\right) \delta 145.6,145.3,140.2,139.8,139.4,139.3,139.0,133.6,131.8,131.7$, 129.1, 128.4, 127.7, 127.5, 127.0, 126.9, 126.6, 126.5, 71.60, 71.55, 7.3, 6.7 ppm, 2 signals coincide; IR (ATR): v $3029(\mathrm{w}), 2952(\mathrm{~m}), 2908(\mathrm{w}), 2873(\mathrm{~m}), 1481(\mathrm{~m}), 1455(\mathrm{w}), 1393(\mathrm{w}), 1237$ (m), 1178 (w), 1144 (m), 1068 (s), 1003 (s), 959 (s), 862 (m), 809 (s) cm ${ }^{-1}$; HRMS (ESI-TOF, positive mode) $\mathrm{m} / \mathrm{z}$ calcd for $\mathrm{C}_{84} \mathrm{H}_{100}{ }^{35} \mathrm{Cl}_{2} \mathrm{O}_{4} \mathrm{Si}_{4}: 1354.6076[\mathrm{M}]^{+}$, found 1354.5940 .

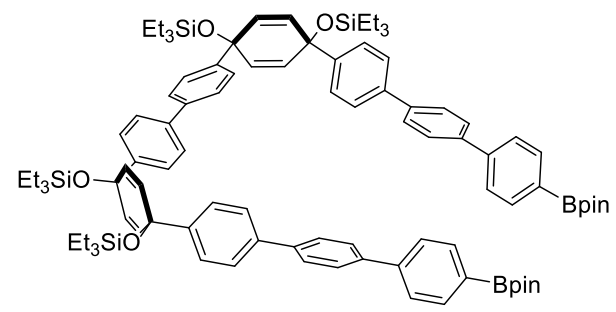

Compound 7. Starting S3 (1.70 g, $1.25 \mathrm{mmol}, 1.00$ equiv.), bis(pinacolato)diboron (1.91 g, 7.52 mmol, 6.00 equiv.), potassium acetate $(738 \mathrm{mg}, 7.52 \mathrm{mmol}, 6.0$ equiv.), palladium(II) acetate (14.1 $\mathrm{mg}, 62.6 \mu \mathrm{mol}, 5.0 \mathrm{~mol} \%$ ) and SPhos (51.4 mg, $0.13 \mathrm{mmol}, 10.0 \mathrm{~mol} \%)$ were added to a flame dried flask and set under $\mathrm{N}_{2}$ atmosphere (potassium acetate was finely ground with a mortar and pestle and oven or flame dried immediately before use for best results). 1,4-Dioxane (anhydrous, $\mathrm{N}_{2}$ sparged for $30 \mathrm{~min}, 25 \mathrm{~mL}, 0.05 \mathrm{M}$ ) was added and the resulting solution was sparged for $1 \mathrm{~h}$ with $\mathrm{N}_{2}$. The reaction was heated to $80^{\circ} \mathrm{C}$ for $12 \mathrm{~h}$ at which point it was cooled to room temperature and filtered over a plug of Celite. The crude mixture was concentrated to afford a colorless viscous oil, which was triturated with cold ethanol to give product 7 as a white solid $(1.80 \mathrm{~g}, 1.17 \mathrm{mmol}$, $93 \%) . R_{\mathrm{f}}=0.36\left(\mathrm{SiO}_{2}, 50 \% \mathrm{CH}_{2} \mathrm{Cl}_{2} /\right.$ hexanes $)$, the product is decomposing on silica; $\mathrm{Mp} 151.7-$ $153.0{ }^{\circ} \mathrm{C}$ (decomp.); ${ }^{1} \mathrm{H}$ NMR $\left(500 \mathrm{MHz}, \mathrm{CDCl}_{3}\right) \delta 7.89$ (d, J=8.1 Hz, 4H), $7.66(\mathrm{~s}, 8 \mathrm{H}), 7.63$ (d, $J=8.1 \mathrm{~Hz}, 4 \mathrm{H}), 7.55(\mathrm{~d}, J=8.5,4 \mathrm{H}), 7.52(\mathrm{~d}, J=8.5,4 \mathrm{H}), 7.43(\mathrm{dd}, J=8.4,6.5 \mathrm{~Hz}, 8 \mathrm{H}), 6.05$ (s, $8 \mathrm{H}), 1.37(\mathrm{~s}, 24 \mathrm{H}), 0.96(\mathrm{dt}, J=7.8,7.8 \mathrm{~Hz}, 38 \mathrm{H}), 0.64(\mathrm{dq}, J=10.8,7.8 \mathrm{~Hz}, 24 \mathrm{H}) \mathrm{ppm} ;{ }^{13} \mathrm{C}$ NMR $\left(126 \mathrm{MHz} \mathrm{CDCl}_{3}\right) \delta 145.5,145.3,143.5,140.2,140.0,139.8,139.6,135.5,131 \mathrm{fzz} .73,131.71$, 127.7, 127.6, 127.0, 126.9, 126.6, 126.52, 126.48, 84.0, 71.60, 71.56, 25.1, 7.3, 6.7 ppm, three signals coincide; IR (ATR): ṽ 3029 (w), 2952 (m), 2909 (w), 2874 (m), 1609 (w), 1490 (w), 1456 (w), $1397(\mathrm{~m}), 1358$ (s), 1320 (w), 1238 (w), 1178 (w), $1144(\mathrm{~m}), 1070$ (s), 1004 (s), 960 (s), 859 (m), 813 (s) $\mathrm{cm}^{-1}$; HRMS (ESI-TOF, positive mode) $\mathrm{m} / z$ calcd for $\mathrm{C}_{96} \mathrm{H}_{124}{ }^{11} \mathrm{~B}_{2} \mathrm{O}_{8} \mathrm{Si}_{4}: 1538.8559$ $[\mathrm{M}]^{+}$, found 1538.8323 . 


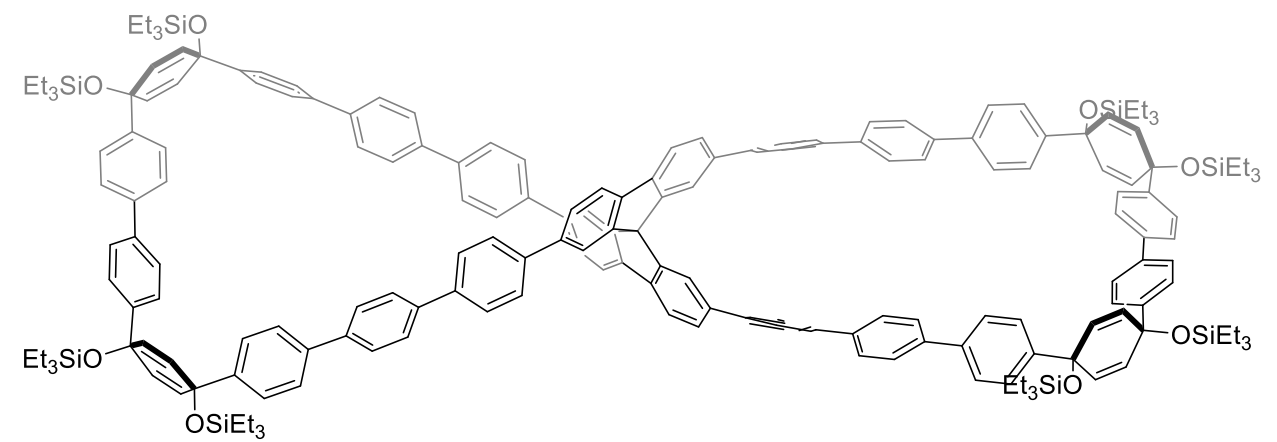

Compound 11. 2,2',7,7'-Tetrabromo-9,9'-spirobifluorene ( $0.31 \mathrm{~g}, 0.49 \mathrm{mmol}, 1.00$ equiv.), compound 7 (1.65 g, $1.07 \mathrm{mmol}, 2.20$ equiv.), and (2-dicyclohexylphosphino-2',6'dimethoxybiphenyl)[2-(2'-amino-1,1'-biphenyl)]palladium(II)methanesulfonate (SPhos Pd G3, $19.0 \mathrm{mg}, 24.4 \mu \mathrm{mol}, 5.0 \mathrm{~mol} \%$ ) were added to 1,4-dioxane (anhydrous, $\mathrm{N}_{2}$ sparged for $30 \mathrm{~min}$, $97.5 \mathrm{~mL}, 0.005 \mathrm{M})$ and the resulting solution was sparged with $\mathrm{N}_{2}$ for $1 \mathrm{~h}$. The reaction mixture was heated to $80^{\circ} \mathrm{C}$ followed by addition of aqueous $\mathrm{K}_{3} \mathrm{PO}_{4}$ solution $\left(\mathrm{N}_{2}\right.$ sparged for $1 \mathrm{~h}, 2.0 \mathrm{M}$, $9.8 \mathrm{~mL}$, exc.) via syringe. The reaction was stirred at $80^{\circ} \mathrm{C}$ for $16 \mathrm{~h}$ before it was allowed to cool to room temperature and water was added $(100 \mathrm{~mL})$. The product was extracted with dichloromethane $(3 \times 50 \mathrm{~mL})$. The combined organic fractions were washed with water $(1 \times 50$ $\mathrm{mL})$, and brine $(1 \times 50 \mathrm{~mL})$, dried over sodium sulfate and the solvent was removed. The resulting brownish crude product was purified by column chromatography $\left(\mathrm{SiO}_{2}, 0-10 \% \mathrm{EtOAc/hexanes)}\right.$ to yield product 11 together with small amounts of polymeric material as a white solid $(0.49 \mathrm{~g}, 0.17$ $\mathrm{mmol}, 35 \%)$. Analytically pure samples can be obtained by gel permeation chromatography $\left(\mathrm{CHCl}_{3}\right) . R_{\mathrm{f}}=0.44\left(\mathrm{SiO}_{2}, 50 \% \mathrm{CH}_{2} \mathrm{Cl}_{2} /\right.$ hexanes$) ; \mathrm{Mp}>300{ }^{\circ} \mathrm{C} ;{ }^{1} \mathrm{H}$ NMR $\left(500 \mathrm{MHz}, \mathrm{CDCl}_{3}\right) \delta 8.00$ (d, $J=7.9 \mathrm{~Hz}, 4 \mathrm{H}$ ), 7.76 (dd, $J=7.9,1.7 \mathrm{~Hz}, 4 \mathrm{H}), 7.63(\mathrm{~d}, J=8.3 \mathrm{~Hz}, 8 \mathrm{H}), 7.60(\mathrm{~d}, J=8.3 \mathrm{~Hz}$, $8 \mathrm{H}), 7.58-7.53(\mathrm{~m}, 22 \mathrm{H}), 7.52-7.47(\mathrm{~m}, 22 \mathrm{H}), 7.43(\mathrm{br}, \mathrm{s}, 4 \mathrm{H}), 7.05(\mathrm{~d}, J=1.7 \mathrm{~Hz}, 4 \mathrm{H}), 6.08-$ $6.00(\mathrm{~m}, 16 \mathrm{H}), 0.96(\mathrm{t}, J=7.9 \mathrm{~Hz}, 72 \mathrm{H}), 0.64(\mathrm{q}, J=7.9 \mathrm{~Hz}, 48 \mathrm{H}) \mathrm{ppm} ;{ }^{13} \mathrm{C} \mathrm{NMR}\left(126 \mathrm{MHz}, \mathrm{CDCl}_{3}\right)$ $\delta$ 150.6, 145.61, 145.56, 141.0, $140.7139 .9,139.7,139.6,139.53,139.51,139.46,132.2,132.0$, $131.5,130.9,130.8,127.5,127.41,127.38,127.35,126.90,126.88,126.22,126.19,123.8,120.5$, 71.4, 71.0, 67.0, 7.10, 7.08, 6.49, 6.46 ppm; IR (ATR): $\tilde{v} 3029(\mathrm{w}), 2950(\mathrm{~m}), 2907(\mathrm{w}), 2874(\mathrm{~m})$, $1489(\mathrm{w}), 1462(\mathrm{~m}), 1398(\mathrm{w}), 1236(\mathrm{~m}), 1177(\mathrm{~m}), 1062(\mathrm{~s}), 1004(\mathrm{~s}), 958(\mathrm{~s}), 863(\mathrm{~m}), 809(\mathrm{~s})$ $\mathrm{cm}^{-1}$; HR-MS (MALDI, DHB, positive mode) $\mathrm{m} / z$ calcd for $\mathrm{C}_{193} \mathrm{H}_{213} \mathrm{O}_{8} \mathrm{Si}_{8}: 2882.4415[\mathrm{M}+\mathrm{H}]^{+}$; found 2882,4408 .

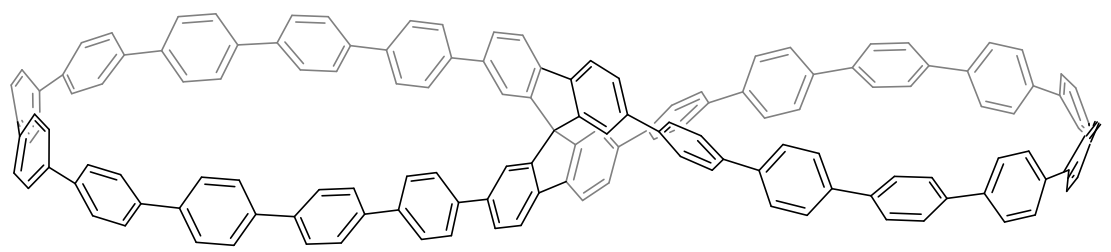

Compound 3. To a solution of compound $11(0.47 \mathrm{~g}, 0.16 \mathrm{mmol}, 1.00$ equiv.) in THF ( $8.0 \mathrm{~mL}$, $0.02 \mathrm{M}$ ) tetrabutylammonium fluoride (TBAF, $1.0 \mathrm{M}, 2.0 \mathrm{~mL}, 2.0 \mathrm{mmol}, 12.0$ equiv.) was added at room temperature and the reaction mixture stirred for $4 \mathrm{~h}$. Water $(10 \mathrm{~mL})$ was added and the THF was almost completely evaporated under reduced pressure. The resulting solid was finely dispersed in an ultrasonication bath, filtered off, and thoroughly washed with water and $\mathrm{CH}_{2} \mathrm{Cl}_{2}$ to afford the deprotected macrocycle as an off-white solid $(0.310 \mathrm{~g}, 0.16 \mathrm{mmol}, 97 \%)$. 
In the next step, to a solution of $\mathrm{SnCl}_{2} \cdot 2 \mathrm{H}_{2} \mathrm{O}(0.17 \mathrm{~g}, 0.76 \mathrm{mmol}, 6.00$ equiv.) in THF ( $4.0 \mathrm{~mL}, 0.2$ $\mathrm{M})$ was added concentrated aqueous $\mathrm{HCl}(124 \mu \mathrm{L}, 1.48 \mathrm{mmol}, 11.7$ equiv.) at room temperature and the resulting solution was stirred for 15 min under nitrogen atmosphere. The resulting mixture was added to a dispersion of the as prepared deprotected macrocycle $(0.25 \mathrm{~g}, 0.12 \mathrm{mmol}, 1.00$ equiv.) in THF (13 mL, $0.01 \mathrm{M}$ ) and the mixture was stirred for $1 \mathrm{~h}$ at room temperature. The reaction mixture was concentrated under reduced pressure (ca. $8 \mathrm{~mL}$ ) and directly subjected to column chromatography (basic alumina, $50 \% \mathrm{CH}_{2} \mathrm{Cl}_{2} /$ hexanes) to yield product 3 as a slightly yellowish, blue fluorescent solid $(89.0 \mathrm{mg}, 48.5 \mu \mathrm{mol}, 38 \%)$. The product can be stored under ambient conditions for months without noticeable decomposition. $R_{\mathrm{f}}=0.22\left(\mathrm{SiO}_{2}, 50 \%\right.$ $\mathrm{CH}_{2} \mathrm{Cl}_{2} /$ hexanes); $\mathrm{Mp}>300^{\circ} \mathrm{C} ; \delta^{1} \mathrm{H}$ NMR $\left(600 \mathrm{MHz}, \mathrm{CDCl}_{3}\right) \delta 7.98(\mathrm{~d}, J=8.1 \mathrm{~Hz}, 4 \mathrm{H}), 7.73(\mathrm{~d}$, $J=8.1 \mathrm{~Hz}, 4 \mathrm{H}), 7.65-7.48(\mathrm{~m}, 80 \mathrm{H}), 7.12(\mathrm{~s}, 4 \mathrm{H}) \mathrm{ppm} ;{ }^{13} \mathrm{C} \mathrm{NMR}\left(126 \mathrm{MHz}, \mathrm{CDCl}_{3}\right) \delta 150.0$, $141.0,140.3$, 139.5, 139.4, 139.3, 139.2, 139.0, 138.7, 138.61, 138.58, 138.40, 138.38, 138.3, 127.70, 127.66, 127.65, 127.62, 127.56, 127.54, 127.45, 127.40, 127.37, 127.3, 122.1, 120.7, 66.9 ppm; IR (ATR): $\tilde{v} 3023$ (w), 2951 (w), 2920 (w), 2867 (w), 1593 (w) $1483(\mathrm{~m}), 1473(\mathrm{~m}), 1390$ (w), $1244(\mathrm{w}), 1111(\mathrm{w}), 1001(\mathrm{~m}), 806(\mathrm{~s}) \mathrm{cm}^{-1}$; HR-MS (MALDI, DCTB, positive mode) $\mathrm{m} / \mathrm{z}$ calcd for $\mathrm{C}_{145} \mathrm{H}_{92}: 1832.7199[\mathrm{M}]^{+}$, found 1832.7298 .

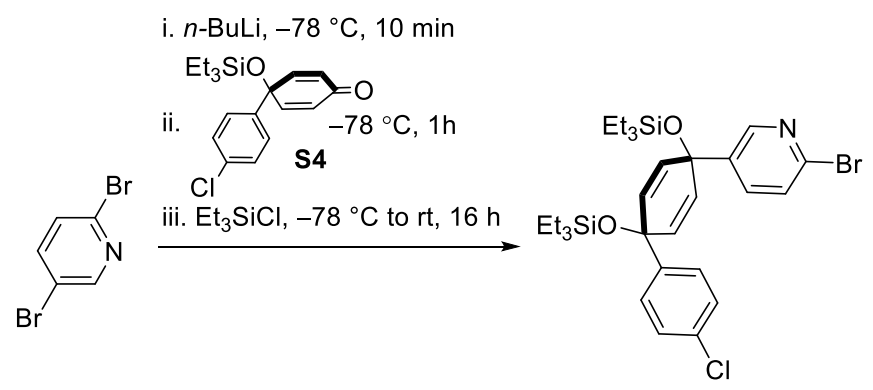

Compound S5. 2,5-Dibromopyridine $(7.78 \mathrm{~g}, 32.8 \mathrm{mmol}, 1.1$ equiv.) was dissolved in THF $(140 \mathrm{~mL}, 0.2 \mathrm{M})$ and the solution cooled to $-78^{\circ} \mathrm{C}$. $n$-Butyllithium $(2.5 \mathrm{M}$ in hexanes, $12.5 \mathrm{~mL}$, $31.4 \mathrm{mmol}, 1.05$ equiv.) was added dropwise over $5 \mathrm{~min}$. After stirring the reaction mixture for another $10 \mathrm{~min}$, compound S4 ${ }^{[2]}(10.0 \mathrm{~g}, 29.9 \mathrm{mmol}, 1.0$ equiv.) dissolved in THF (20 mL) was added dropwise via syringe at $-78{ }^{\circ} \mathrm{C}$. The resulting reaction mixture was stirred for $1 \mathrm{~h}$ at $-78{ }^{\circ} \mathrm{C}$. Chlorotriethylsilane $(7.5 \mathrm{~mL}, 44.8 \mathrm{mmol}, 1.5$ equiv.) was added via syringe and the reaction was allowed to warm to roomt temperature overnight. Water $(100 \mathrm{~mL})$ was added and the reaction mixture was extracted with EtOAc $(3 \times 100 \mathrm{~mL})$. The combined organic fractions were washed with water $(2 \times 50 \mathrm{~mL})$ and brine $(100 \mathrm{~mL})$, dried over sodium sulfate, and the solvent was removed under reduced pressure. The crude product was purified by column chromatography $\left(\mathrm{SiO}_{2}, 10 \% \mathrm{EtOAc/hexanes)}\right.$ to give product $\mathbf{S} 5$ as a highly viscous colorless oil that is solidifying overnight $(15.0 \mathrm{~g}, 83 \%) . R_{\mathrm{f}}=0.55\left(\mathrm{SiO}_{2}, 10 \% \mathrm{EtOAc/hexanes}\right) ;{ }^{1} \mathrm{H} \mathrm{NMR}\left(500 \mathrm{MHz}, \mathrm{CDCl}_{3}\right) \delta 8.31$ $(\mathrm{d}, J=2.5 \mathrm{~Hz}, 1 \mathrm{H}), 7.42(\mathrm{dd}, J=8.5,2.5 \mathrm{~Hz}, 1 \mathrm{H}), 7.35(\mathrm{~d}, J=8.5 \mathrm{~Hz}, 1 \mathrm{H}), 6.09(\mathrm{~d}, J=9.8 \mathrm{~Hz}$, $2 \mathrm{H}), 5.94(\mathrm{~d}, J=9.8 \mathrm{~Hz}, 2 \mathrm{H}), 0.97(\mathrm{t}, J=7.9 \mathrm{~Hz}, 9 \mathrm{H}), 0.92(\mathrm{t}, J=7.9 \mathrm{~Hz}, 9 \mathrm{H}), 0.66(\mathrm{q}, J=7.9 \mathrm{~Hz}$, $6 \mathrm{H}), 0.58(\mathrm{q}, J=7.9 \mathrm{~Hz}, 6 \mathrm{H}) \mathrm{ppm} ;{ }^{13} \mathrm{C}$ NMR $\left(126 \mathrm{MHz}, \mathrm{CDCl}_{3}\right) \delta 148.4,144.1,141.00,140.95$, 136.3, 133.6, 132.3, 130.7, 128.71, 127.5, 127.2, 70.8, 70.2, 7.09, 7.07, 6.54, 6.49 ppm; IR (ATR): v $3034(w), 2953(\mathrm{~m}), 2909(\mathrm{w}), 2873(\mathrm{~m}), 1573(\mathrm{w}), 1558(\mathrm{w}), 1460(\mathrm{~m}), 1400(\mathrm{w}), 1360(\mathrm{w}), 1236$ (m), $1185(\mathrm{~m}), 1083$ (s), 1014 (s), 958 (s), $872(\mathrm{w}), 855(\mathrm{~m}), 821(\mathrm{~m}) \mathrm{cm}^{-1}$; HRMS (ESI-TOF, positive mode) $\mathrm{m} / \mathrm{z}$ calcd for $\mathrm{C}_{29} \mathrm{H}_{42}{ }^{79} \mathrm{Br}^{35} \mathrm{CINO}_{2} \mathrm{Si}_{2}: 606.1626[\mathrm{M}+\mathrm{H}]^{+}$, found 606.1645 . 


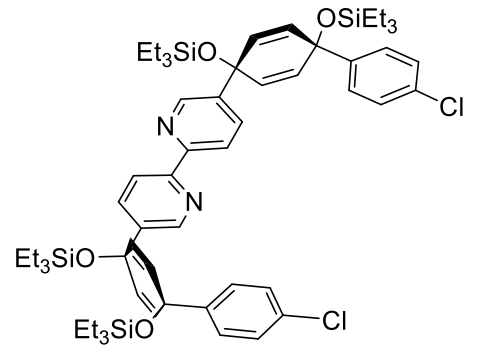

Compound S6. Ni( $\left(\mathrm{PPh}_{3}\right)_{2} \mathrm{Br}_{2}(4.59 \mathrm{~g}, 6.18 \mathrm{mmol}, 0.75$ equiv.), $\mathrm{Mn}(0.68 \mathrm{~g}, 12.4 \mathrm{mmol}, 1.5$ equiv.), $\mathrm{PPh}_{3}\left(3.24 \mathrm{~g}, 12.4 \mathrm{mmol} 1.5\right.$ equiv.), and $\mathrm{NEt}_{4} \mathrm{l}(1.60 \mathrm{~g}, 6.18 \mathrm{mmol}, 0.75 \mathrm{mmol}$ ) were added to a flame dried flask and evacuated and back-filled with $\mathrm{N}_{2}(5 \times)$, followed by the addition of $\mathrm{N}_{2}$ sparged DMF $(40 \mathrm{~mL}, 0.15 \mathrm{M})$. The resulting suspension was sonicated until the color turned from green to dark red $(15 \mathrm{~min})$ at which point the suspension was stirred at $60^{\circ} \mathrm{C}$ for 1 hour. S5 $(5.0$ $\mathrm{g}, 8.23 \mathrm{mmol}, 1.0$ equiv.) in nitrogen sparged DMF $(55 \mathrm{~mL}, 0.15 \mathrm{M})$ was added to this mix via syringe and the reaction mixture was stirred one hour at room temperature. A solution of aqueous ammonia (18\%) and EDTA (saturated, $70 \mathrm{~mL}$ ) together with EtOAc $(100 \mathrm{~mL})$ was added and the mixture was vigorously stirred for $30 \mathrm{~min}$. The slightly bluish aqueous phase was extracted with EtOAC $(2 \times 100 \mathrm{~mL})$ and the combined organic fractions were washed with $\mathrm{LiCl}(5 \%, 2 \times 50 \mathrm{~mL})$, and brine $(100 \mathrm{~mL})$, and dried over sodium sulfate. After solvent removal under reduced pressure, the obtained orange oil was purified by column chromatography $\left(\mathrm{SiO}_{2}\right.$, from $\mathrm{CH}_{2} \mathrm{Cl}_{2} / \mathrm{Hex}, 0-50 \%$ $\mathrm{DCM} / \mathrm{Hex})$ to give product $\mathbf{S 6}$ as a white solid $(1.77 \mathrm{~g}, 41 \%) . R_{\mathrm{f}}=0.79\left(\mathrm{SiO}_{2}, 10 \%\right.$ EtOAc/hexanes); Mp 211.9-212.4 ${ }^{\circ} \mathrm{C} ;{ }^{1} \mathrm{H} \mathrm{NMR}\left(500 \mathrm{MHz}, \mathrm{CDCl}_{3}\right) \delta 8.69(\mathrm{~d}, J=2.4 \mathrm{~Hz}, 2 \mathrm{H}), 8.25$ (d, J = 8.3 Hz, 2H), $7.62(\mathrm{dd}, J=8.3,2.4 \mathrm{~Hz}, 2 \mathrm{H}), 7.26(\mathrm{~s}, 8 \mathrm{H}), 6.04(\mathrm{~d}, J=2.4 \mathrm{~Hz}, 8 \mathrm{H}), 0.95(\mathrm{q}, J$ $=8.1 \mathrm{~Hz}, 36 \mathrm{H}), 0.62(\mathrm{dq}, J=17.8,8.1 \mathrm{~Hz}, 24 \mathrm{H}) \mathrm{ppm} ;{ }^{13} \mathrm{C} \mathrm{NMR}\left(126 \mathrm{MHz}, \mathrm{CDCl}_{3}\right) \delta 155.0,147.5$, 144.4, 141.5 134.5, 133.4, 132.1, 131.1, 128.7, 127.3, 120.6, 71.1, 70.5, 7.20, 7.19, 6.62, 6.61 ppm; IR (ATR): $\tilde{v} 3035(\mathrm{w}), 2951(\mathrm{~m}), 2908(\mathrm{w}), 2873(\mathrm{~m}), 1590(\mathrm{w}), 1549(\mathrm{w}), 1482(\mathrm{w}), 1458(\mathrm{~m})$, $1397(\mathrm{w}), 1238(\mathrm{~m}), 1188(\mathrm{~m}), 1093(\mathrm{~s}), 1056(\mathrm{~s}), 1014(\mathrm{~s}), 956(\mathrm{~s}), 874(\mathrm{w}), 856(\mathrm{~m}), 821(\mathrm{w}) \mathrm{cm}^{-}$ 1; HRMS (ESI-TOF, positive mode) $\mathrm{m} / \mathrm{z}$ calcd for $\mathrm{C}_{58} \mathrm{H}_{83}{ }^{35} \mathrm{Cl}_{2} \mathrm{~N}_{2} \mathrm{O}_{4} \mathrm{Si}_{4}: 1053.4807[\mathrm{M}+\mathrm{H}]^{+}$, found 1053.4712 .

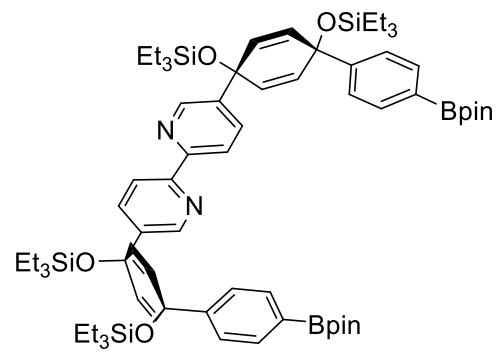

Compound 8. Starting S6 (1.70 g, $1.61 \mathrm{mmol}, 1.00$ equiv.), bis(pinacolato)diboron (1.64 g, 0.81 mmol, 4.00 equiv.), potassium acetate ( $633 \mathrm{mg}, 6.44 \mathrm{mmol}, 4.0$ equiv.), palladium(II) acetate (18.1 $\mathrm{mg}, 80.6 \mu \mathrm{mol}, 5.0 \mathrm{~mol} \%$ ) and SPhos ( $82.7 \mathrm{mg}, 0.20 \mathrm{mmol}, 13.0 \mathrm{~mol} \%$ ) were added to a flame dried flask and set under $\mathrm{N}_{2}$ atmosphere (potassium acetate was finely ground with a mortar and pestle and oven or flame dried immediately before use). 1,4-Dioxane (anhydrous, $\mathrm{N}_{2}$ sparged for $30 \mathrm{~min}, 32 \mathrm{~mL}, 0.05 \mathrm{M}$ ) was added and the resulting solution was sparged for $1 \mathrm{~h}$ with $\mathrm{N}_{2}$. The reaction was heated to $80^{\circ} \mathrm{C}$ for $16 \mathrm{~h}$ at which point it was cooled to room temperature and filtered over a plug of Celite. The crude mixture was concentrated to afford a colorless viscous oil, which was triturated with cold ethanol to give product 8 as a off-white solid $(1.88 \mathrm{~g}, 1.52 \mathrm{mmol}, 94 \%)$. $R_{\mathrm{f}}$ 
$=0.36\left(\mathrm{SiO}_{2}, 50 \% \mathrm{CH}_{2} \mathrm{Cl}_{2} /\right.$ hexanes$)$, the product is decomposing on silica; $\mathrm{Mp} 151.7-153.0{ }^{\circ} \mathrm{C}$ (decomp.);

$R_{\mathrm{f}}=0.38\left(\mathrm{SiO}_{2}, 10 \%\right.$ EtOAc/hexanes); $\mathrm{Mp} 196.1{ }^{\circ} \mathrm{C} ;{ }^{1} \mathrm{H} \mathrm{NMR}\left(500 \mathrm{MHz}, \mathrm{CDCl}_{3}\right) \delta 8.74$ (d, $J=2.2$ $\mathrm{Hz}, 2 \mathrm{H}$ ), $8.22(\mathrm{~d}, J=8.3 \mathrm{~Hz}, 2 \mathrm{H}), 7.73(\mathrm{~d}, J=8.2 \mathrm{~Hz}, 4 \mathrm{H}), 7.58(\mathrm{dd}, J=8.3,2.2 \mathrm{~Hz}, 2 \mathrm{H}), 7.35(\mathrm{~d}$, $J=8.2 \mathrm{~Hz}, 4 \mathrm{H}), 6.08(\mathrm{~d}, J=10.2 \mathrm{~Hz}, 4 \mathrm{H}), 6.00(\mathrm{~d}, J=10.2 \mathrm{~Hz}, 4 \mathrm{H}), 1.34(\mathrm{~s}, 24 \mathrm{H}), 0.96$ (t, $J=8.0$ $\mathrm{Hz}, 18 \mathrm{H}$ ), $0.92(\mathrm{t}, J=7.9 \mathrm{~Hz}, 18 \mathrm{H}), 0.65(\mathrm{q}, J=8.0 \mathrm{~Hz}, 12 \mathrm{H}), 0.58(\mathrm{q}, J=7.9 \mathrm{~Hz}, 12 \mathrm{H}) \mathrm{ppm} ;{ }^{13} \mathrm{C}$ NMR $\left(126 \mathrm{MHz}, \mathrm{CDCl}_{3}\right) \delta{ }^{13} \mathrm{C} \mathrm{NMR}\left(126 \mathrm{MHz}, \mathrm{CDCl}_{3}\right) \delta 154.9,148.9,147.5,141.5,135.1,134.5$, 132.3, 130.9, 125.9, 125.2, 120.5, 83.9, 71.5, 70.7, 25.1, 7.22, 7.20, 6.64, 6.62 ppm; IR (ATR): $\tilde{v}$ $3032(\mathrm{w}), 2954(\mathrm{~m}), 2910(\mathrm{w}), 2875(\mathrm{~m}), 1609(\mathrm{~m}), 1590(\mathrm{w}), 1546(\mathrm{w}), 1398(\mathrm{w}), 1359(\mathrm{~s}), 1322$ $(\mathrm{w}), 1271(\mathrm{w}), 1239(\mathrm{~m}), 1188(\mathrm{w}), 1145(\mathrm{~m}), 1068(\mathrm{~s}), 1004(\mathrm{~m}), 955(\mathrm{~s}), 879(\mathrm{w}), 859(\mathrm{~m}), 821$ (w) $\mathrm{cm}^{-1}$; HRMS (ESI-TOF, positive mode) $\mathrm{m} / \mathrm{z}$ calcd for $\mathrm{C}_{70} \mathrm{H}_{106}{ }^{11} \mathrm{~B}_{2} \mathrm{~N}_{2} \mathrm{O}_{8} \mathrm{Si}_{4}: 1236.7212[\mathrm{M}+\mathrm{H}]^{+}$, found 1236.7157 .

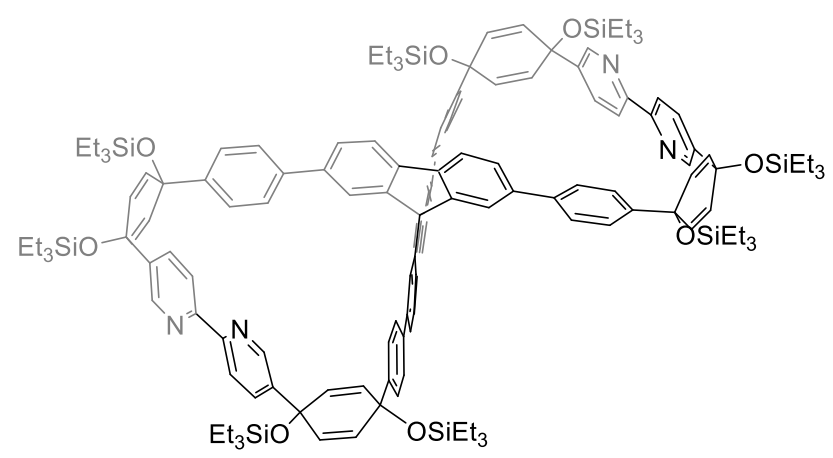

Compound 12. 2,2',7,7'-Tetrabromo-9,9'-spirobifluorene ( $0.28 \mathrm{~g}, 0.44 \mathrm{mmol}, 1.00$ equiv.), compound 8 (1.20 g, $0.97 \mathrm{mmol}, 2.20$ equiv.), and (2-dicyclohexylphosphino-2',6'dimethoxybiphenyl)[2-(2'-amino-1,1'-biphenyl)]palladium(II) methanesulfonate (SPhos Pd G3, $17.3 \mathrm{mg}, 22.2 \mu \mathrm{mol}, 5.0 \mathrm{~mol} \%$ ) were added to 1,4-dioxane (anhydrous, $\mathrm{N}_{2}$ sparged for $30 \mathrm{~min}$, $88.6 \mathrm{~mL}, 0.005 \mathrm{M})$ and the resulting solution was sparged with $\mathrm{N}_{2}$ for $1 \mathrm{~h}$. The reaction mixture was heated to $80^{\circ} \mathrm{C}$ followed by addition of aqueous $\mathrm{K}_{3} \mathrm{PO}_{4}$ solution $\left(\mathrm{N}_{2}\right.$ sparged for $1 \mathrm{~h}, 2.0 \mathrm{M}$, $8.9 \mathrm{~mL}$, exc.) via syringe. The reaction was stirred at $80^{\circ} \mathrm{C}$ for $16 \mathrm{~h}$ before it was allowed to cool to room temperature and water was added $(100 \mathrm{~mL})$. The product was extracted with dichloromethane $(3 \times 50 \mathrm{~mL})$. The combined organic fractions were washed with water $(1 \times 50$ $\mathrm{mL})$, and brine $(1 \times 50 \mathrm{~mL})$, dried over sodium sulfate and the solvent was removed. The resulting off-white crude product was purified by column chromatography $\left(\mathrm{SiO}_{2}, 0-20 \% \mathrm{EtOAc/hexanes)}\right.$ to yield product 12 together with small amounts of polymeric material as a white solid $(0.41 \mathrm{~g}, 0.18$ $\mathrm{mmol}, 41 \%)$. Analytically pure samples can be obtained by gel permeation chromatography $\left(\mathrm{CHCl}_{3}\right) . R_{\mathrm{f}}=0.48\left(\mathrm{SiO}_{2}, 10 \%\right.$ EtOAc/hexanes); $\mathrm{Mp}>300{ }^{\circ} \mathrm{C} ;{ }^{1} \mathrm{H} \mathrm{NMR}\left(500 \mathrm{MHz}, \mathrm{CDCl}_{3}\right) \delta 8.41$ (d, $J=2.3 \mathrm{~Hz}, 4 \mathrm{H}$ ), 7.95 (dd, $J=8.2,4.2 \mathrm{~Hz}, 8 \mathrm{H}), 7.62(\mathrm{dd}, J=8.2,2.3 \mathrm{~Hz}, 4 \mathrm{H}), 7.47$ (d, $J=8.4$ $\mathrm{Hz}, 4 \mathrm{H}$ ), 7.40 (d, $J=8.4 \mathrm{~Hz}, 8 \mathrm{H}$ ), $7.34(\mathrm{~d}, J=8.4 \mathrm{~Hz}, 8 \mathrm{H}$ ), 7.04 (s, 4H), 6.20 (dd, $J=9.9,2.3 \mathrm{~Hz}$, $4 \mathrm{H}), 6.15$ (dd, $J=9.9,2.3 \mathrm{~Hz}, 4 \mathrm{H}), 5.95(\mathrm{dd}, J=10.2,2.1 \mathrm{~Hz}, 4 \mathrm{H}), 5.92(\mathrm{dd}, J=10.2,2.1 \mathrm{~Hz}, 4 \mathrm{H}$ ), $0.98(\mathrm{t}, J=7.9 \mathrm{~Hz}, 36 \mathrm{H}), 0.88(\mathrm{t}, J=8.0 \mathrm{~Hz}, 36 \mathrm{H}), 0.69(\mathrm{q}, J=7.9 \mathrm{~Hz}, 24 \mathrm{H}), 0.52$ (q, $J=8.0 \mathrm{~Hz}$, $24 \mathrm{H}) \mathrm{ppm} ;{ }^{13} \mathrm{C} \mathrm{NMR}\left(126 \mathrm{MHz}, \mathrm{CDCl}_{3}\right) \delta 154.8,149.8,147.2,144.4,141.1,140.90,140.85,140.7$, 134.5, 132.2, 131.9, 131.8, 131.3, 127.7, 127.4, 126.5, 122.9, 120.5, 120.4, 71.2, 71.1, 7.2, 7.1, 6.6 ppm; IR (ATR): $\tilde{v} 3033(\mathrm{w}), 2951(\mathrm{~m}), 2908(\mathrm{w}), 2873(\mathrm{~m}), 1590(\mathrm{w}), 1547(\mathrm{w}), 1460(\mathrm{~m}), 1398$ (w), $1237(\mathrm{~m}), 1189(\mathrm{~m}), 1072(\mathrm{~s}), 1006$ (s), $958(\mathrm{~s}), 857(\mathrm{~m}), 814(\mathrm{~m}) \mathrm{cm}^{-1}$; HR-MS (MALDI, DCTB, positive mode) $\mathrm{m} / z$ calcd for $\mathrm{C}_{141} \mathrm{H}_{177} \mathrm{~N}_{4} \mathrm{O}_{8} \mathrm{Si}_{8}: 2278.1721[\mathrm{M}+\mathrm{H}]^{+}$; found 2278.1698. 


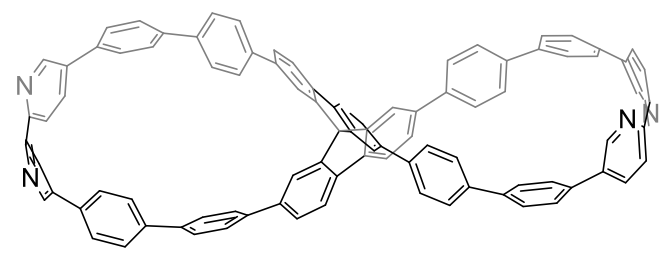

Compound 4. To a solution of compound $12(0.25 \mathrm{~g}, 0.11 \mathrm{mmol}, 1.00$ equiv.) in THF ( $6 \mathrm{~mL}, 0.02$ M) tetrabutylammonium fluoride (TBAF, $1.0 \mathrm{M}, 1.2 \mathrm{~mL}, 2.8 \mathrm{mmol}, 12.0$ equiv.) was added at room temperature and the reaction mixture stirred for $6 \mathrm{~h}$. Water $(20 \mathrm{~mL})$ was added and the THF was almost completely evaporated under reduced pressure. The resulting solid was finely dispersed in an ultrasonication bath, filtered off, and thoroughly washed with water and $\mathrm{CH}_{2} \mathrm{Cl}_{2}$ to afford the deprotected macrocycle as an off-white solid $(0.146 \mathrm{~g}, 0.11 \mathrm{mmol}, 97 \%)$.

In the next step, to a solution of $\mathrm{SnCl}_{2} \cdot 2 \mathrm{H}_{2} \mathrm{O}(0.139 \mathrm{~g}, 0.62 \mathrm{mmol}, 6.00$ equiv. $)$ in THF (10.0 mL, $0.01 \mathrm{M})$ was added concentrated aqueous $\mathrm{HCl}(0.10 \mathrm{~mL}, 1.2 \mathrm{mmol}, 11.7$ equiv.) at room temperature and the solution was stirred for $15 \mathrm{~min}$ under nitrogen atmosphere. The resulting mixture was added to a dispersion of the as prepared deprotected macrocycle $(0.14 \mathrm{~g}, 0.10 \mathrm{mmol}$, 1.00 equiv.) in THF ( $10 \mathrm{~mL}, 0.01 \mathrm{M})$ and the mixture was stirred for $50 \mathrm{~min}$ at room temperature. The reaction mixture was concentrated under reduced pressure (ca. $10 \mathrm{~mL}$ ) and directly subjected to column chromatography (basic alumina, $50-100 \% \mathrm{CH}_{2} \mathrm{Cl}_{2} /$ hexanes) to yield product 4 as a yellow solid $(42.0 \mathrm{mg}, 34.2 \mu \mathrm{mol}, 33 \%)$. The product can be stored under ambient conditions for months without noticeable decomposition. $R_{\mathrm{f}}=0.68$ (basic alumina, $\mathrm{CH}_{2} \mathrm{Cl}_{2}$ ); $\mathrm{Mp}>300{ }^{\circ} \mathrm{C} ;{ }^{1} \mathrm{H}$ $\operatorname{NMR}\left(500 \mathrm{MHz}, \mathrm{CDCl}_{3}\right) \delta 8.92(\mathrm{~d}, J=2.2 \mathrm{~Hz}, 2 \mathrm{H}), 8.70(\mathrm{~d}, J=2.2 \mathrm{~Hz}, 2 \mathrm{H}), 8.06(\mathrm{~d}, J=8.8 \mathrm{~Hz}$, $2 \mathrm{H}), 8.04(\mathrm{~d}, J=8.8 \mathrm{~Hz}, 2 \mathrm{H}), 7.93(\mathrm{~d}, J=8.3 \mathrm{~Hz}, 4 \mathrm{H}), 7.90(\mathrm{dd}, J=8.8,2.0 \mathrm{~Hz}, 2 \mathrm{H}), 7.72-7.68$ $(\mathrm{m}, 6 \mathrm{H}), 7.54-7.50(\mathrm{~m}, 8 \mathrm{H}), 7.43(\mathrm{~d}, J=8.3 \mathrm{~Hz}, 8 \mathrm{H}), 7.38(\mathrm{~d}, J=8.1 \mathrm{~Hz}, 8 \mathrm{H}), 7.31(\mathrm{~d}, J=8.1$ $\mathrm{Hz}, 8 \mathrm{H}), 7.02$ (s, 2H), 7.01 (s, 2H) ppm; ${ }^{13} \mathrm{C} \mathrm{NMR}\left(126 \mathrm{MHz}, \mathrm{CDCl}_{3}\right) \delta$ 150.0, 146.8, 144.5, 140.9, 139.9, 138.9, 138.6, 135.5, 134.6, 131.9, 130.3, 128.10, 127.8, 127.5, 127.4, 127.1, 122.1, 121.8, 121.6, 120.6, 67.3. ppm; IR (ATR): $\tilde{v} 2988$ (w), 2921 (w), 1890 (w), 1604 (w) $1502(w), 1453(\mathrm{~m})$, 1391 (w), 1229 (w), 1060 (m), 809 (s) $\mathrm{cm}^{-1}$; HR-MS (MALDI, DCTB, positive mode) $\mathrm{m} / \mathrm{z}$ calcd for $\mathrm{C}_{93} \mathrm{H}_{56} \mathrm{~N}_{4}: 1228,4505[\mathrm{M}]^{+}$, found 1228,4569. 


\section{Nuclear Magnetic Resonance Spectra}
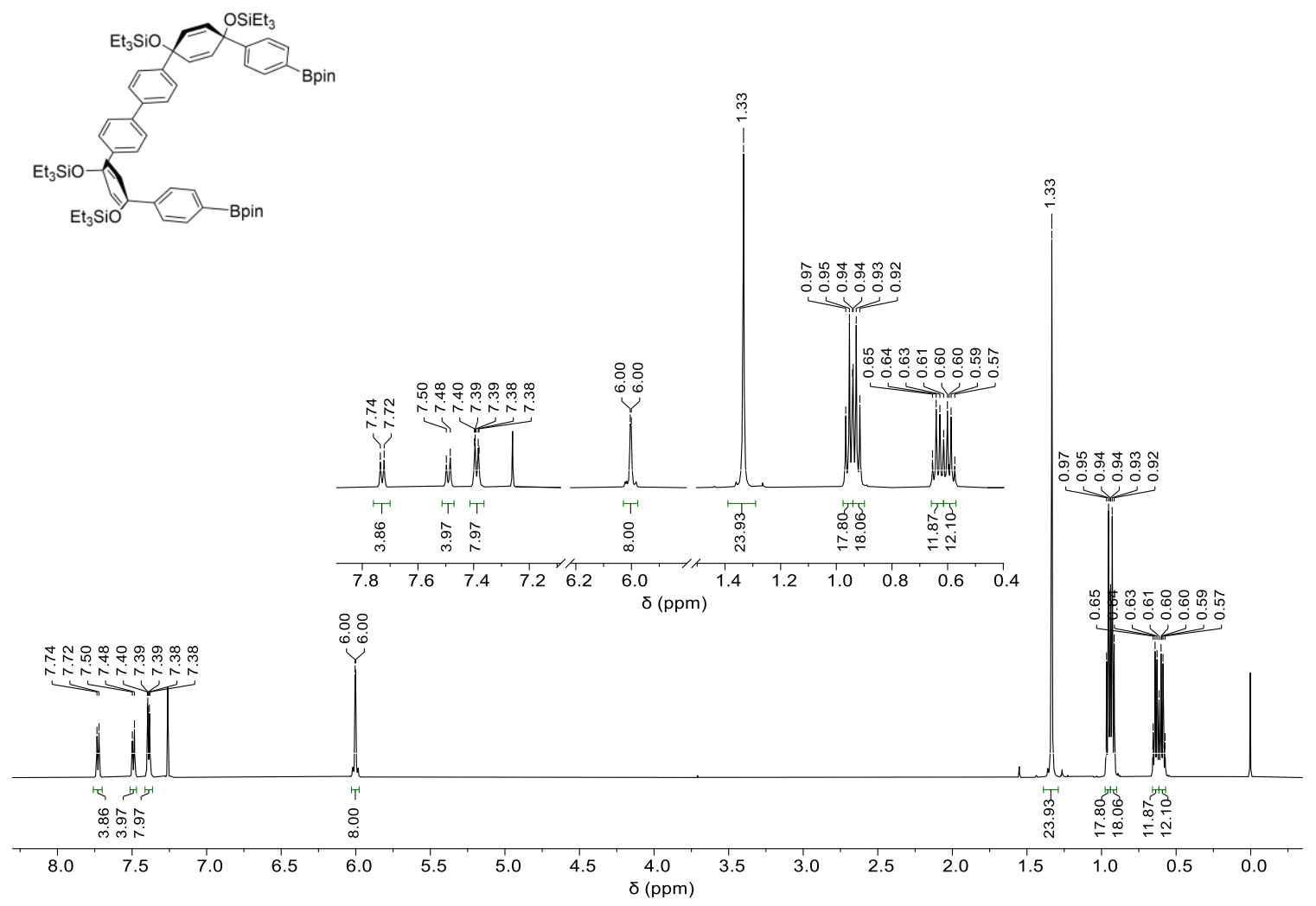

Figure S1: ${ }^{1} \mathrm{H}$ NMR spectrum $(600 \mathrm{MHz})$ of 5 in $\mathrm{CDCl}_{3}$ at room temperature.

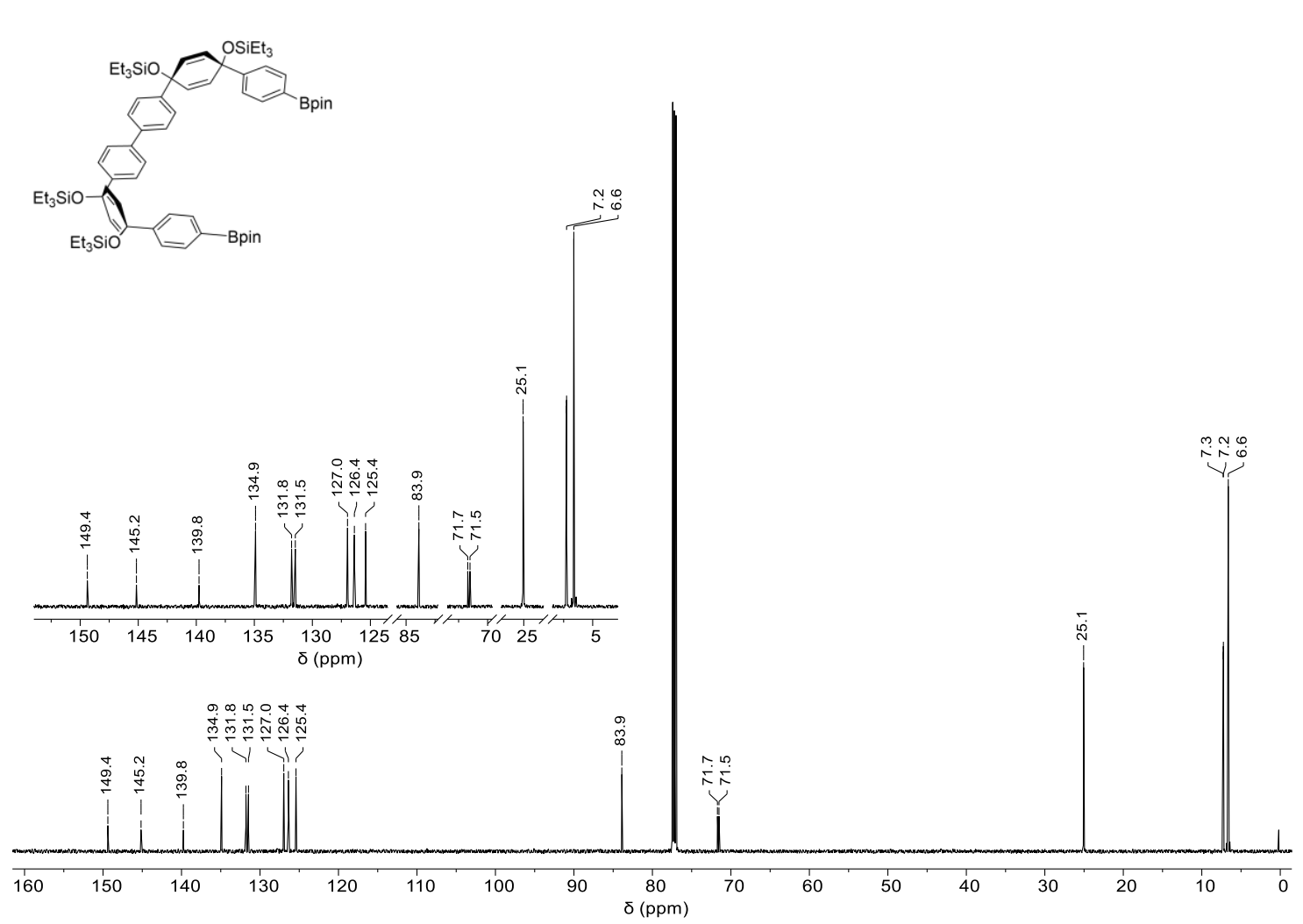

Figure S2: ${ }^{13} \mathrm{C}$ NMR spectrum ( $151 \mathrm{MHz}$ ) of 5 in $\mathrm{CDCl}_{3}$ at room temperature. 

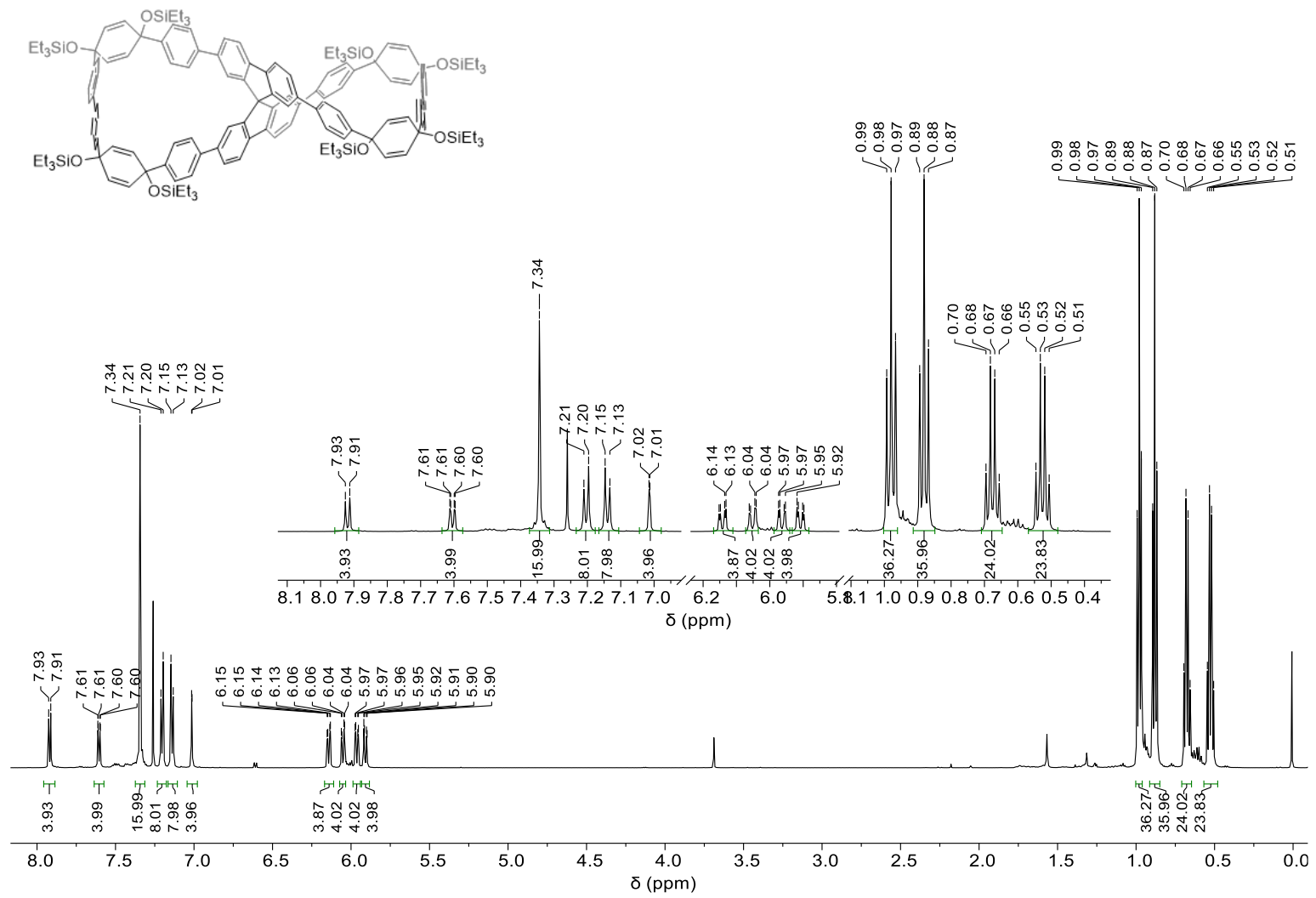

Figure S3: ${ }^{1} \mathrm{H}$ NMR spectrum $(600 \mathrm{MHz})$ of 9 in $\mathrm{CDCl}_{3}$ at room temperature.
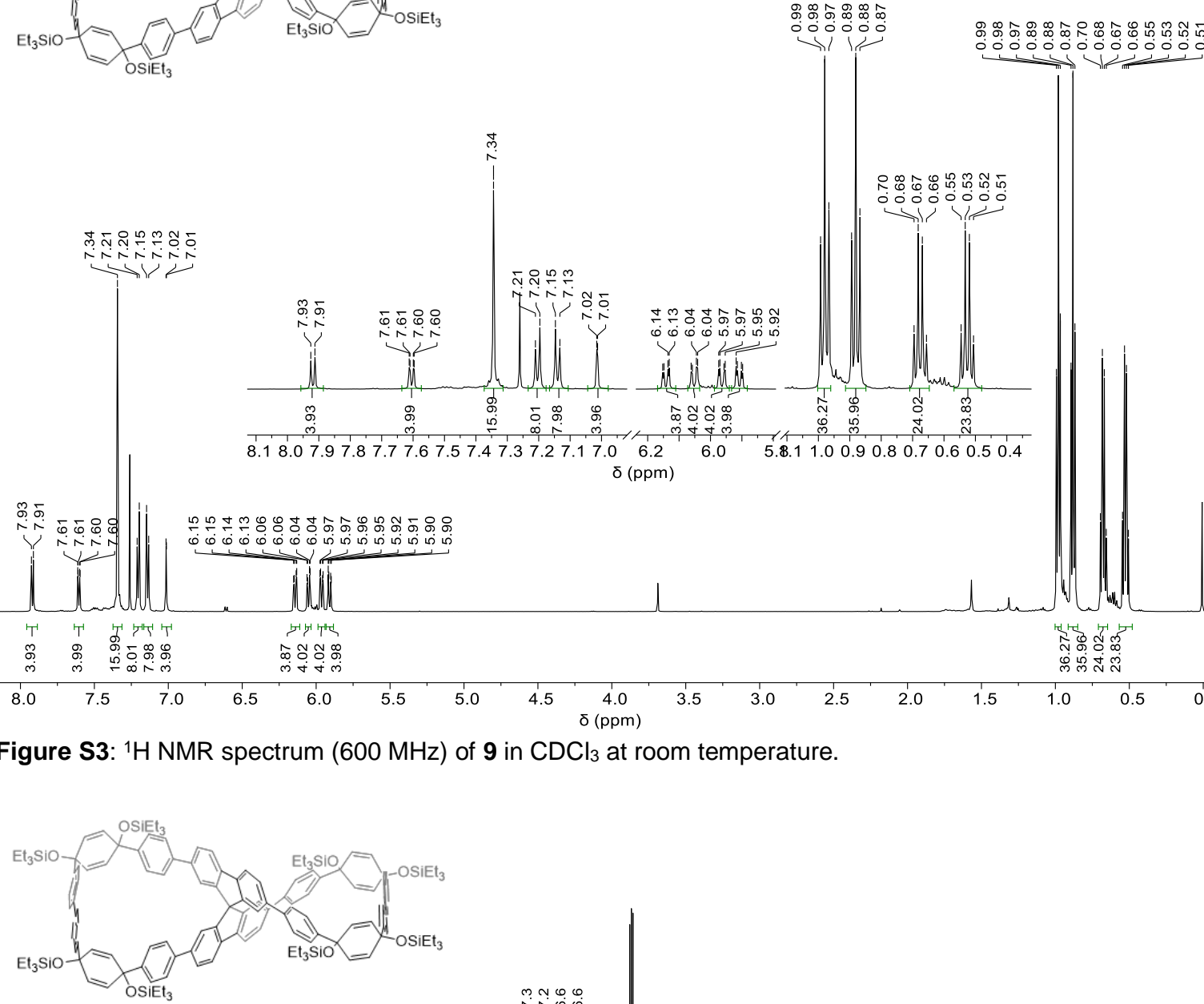

o 00000000000
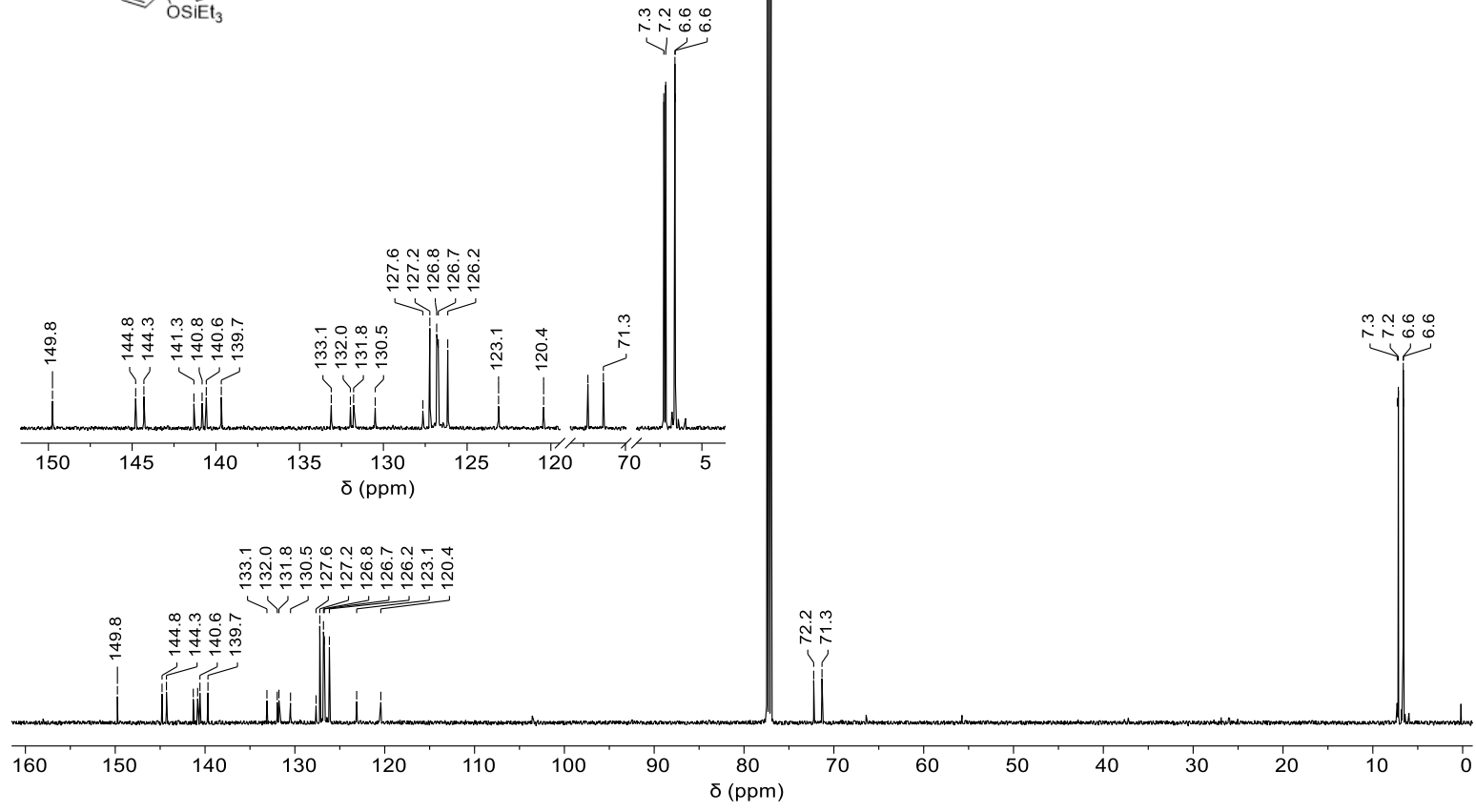

Figure S4: ${ }^{13} \mathrm{C}$ NMR spectrum (151 MHz) of 9 in $\mathrm{CDCl}_{3}$ at room temperature. 


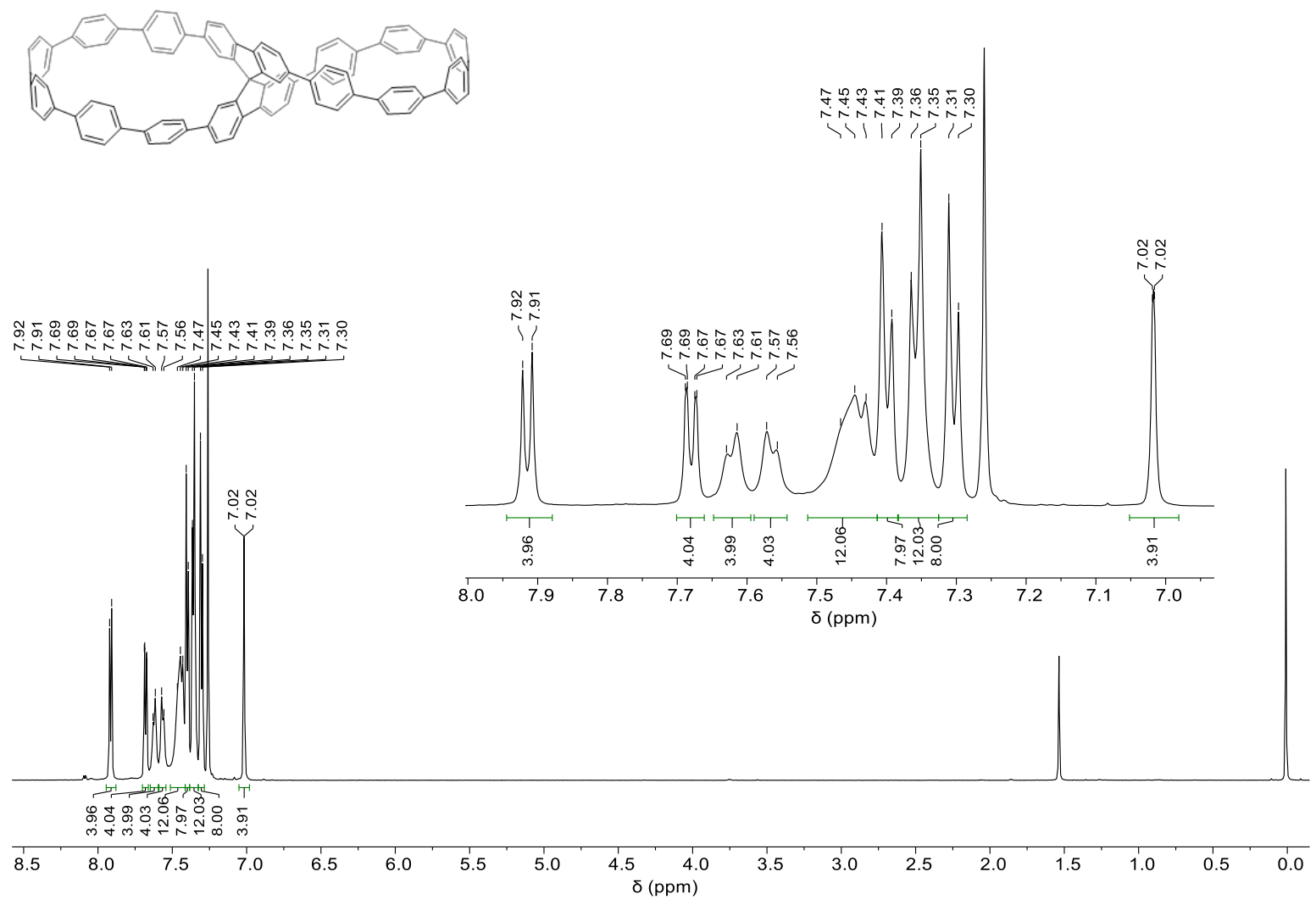

Figure S5: ${ }^{1} \mathrm{H}$ NMR spectrum $(600 \mathrm{MHz})$ of 1 in $\mathrm{CDCl}_{3}$ at room temperature.
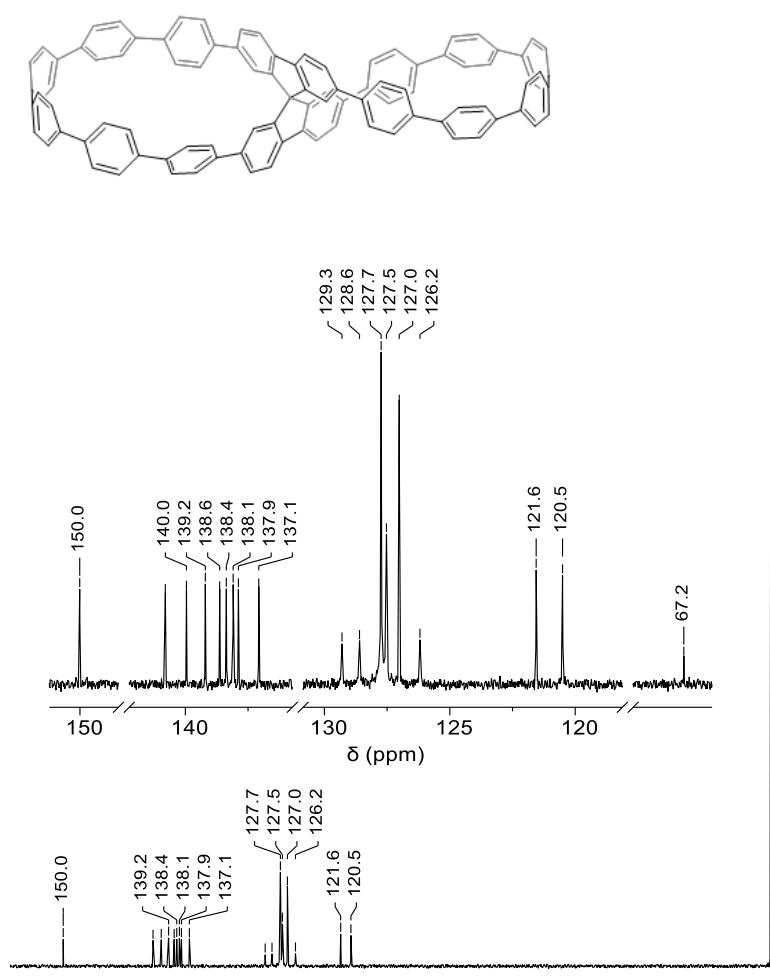

Figure S6: ${ }^{13} \mathrm{C}$ NMR spectrum ( $\left.151 \mathrm{MHz}\right)$ of 1 in $\mathrm{CDCl}_{3}$ at room temperature. 

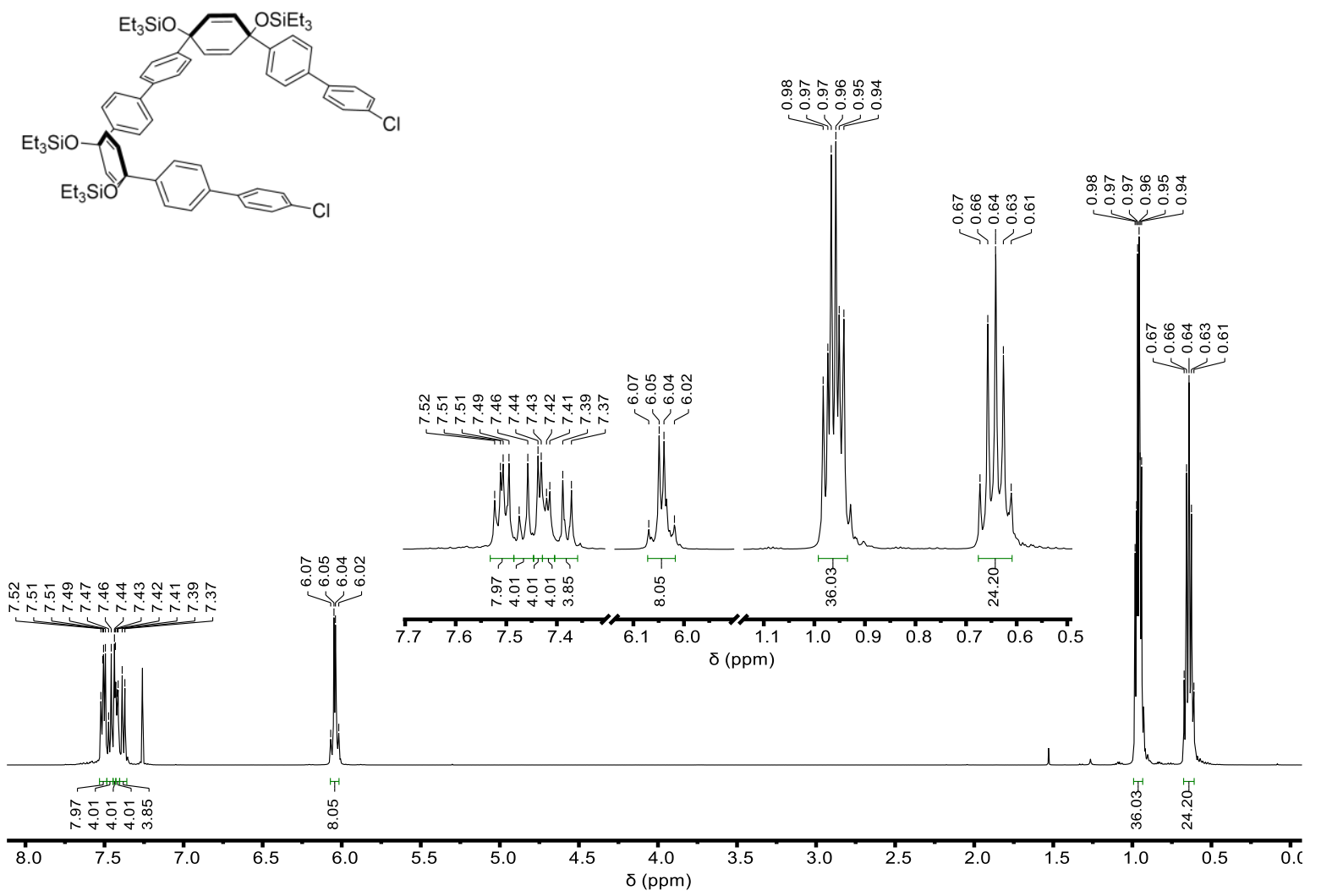

Figure S7: ${ }^{1} \mathrm{H}$ NMR spectrum $(500 \mathrm{MHz})$ of $\mathbf{S} 2$ in $\mathrm{CDCl}_{3}$ at room temperature.
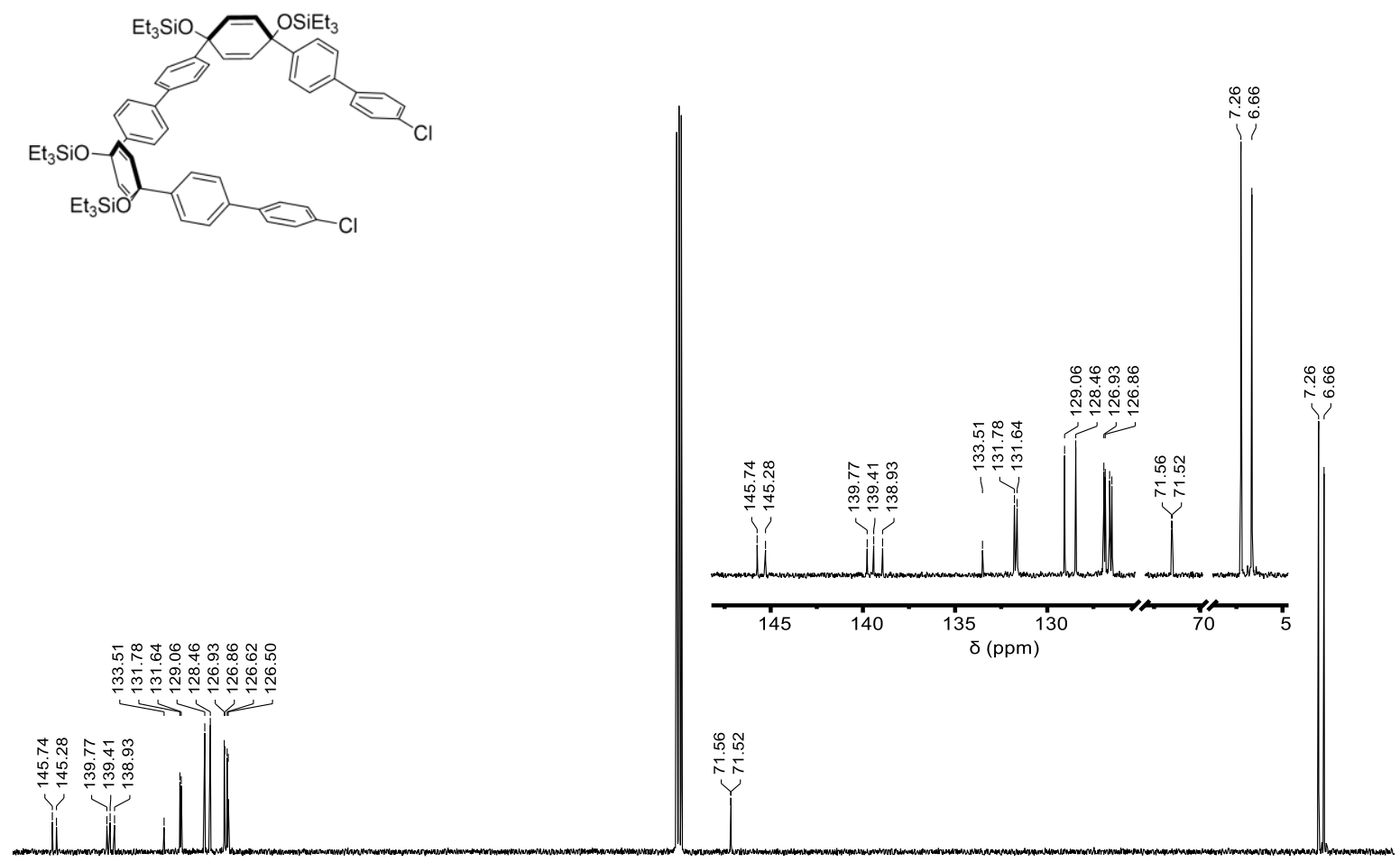

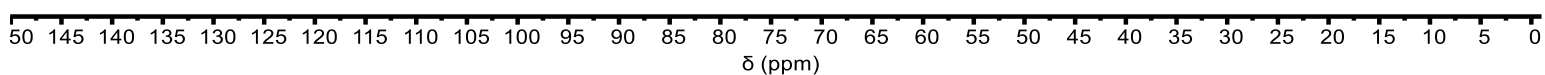

Figure S8: ${ }^{13} \mathrm{C}$ NMR spectrum (126 MHz) of $\mathbf{S} 2$ in $\mathrm{CDCl}_{3}$ at room temperature. 

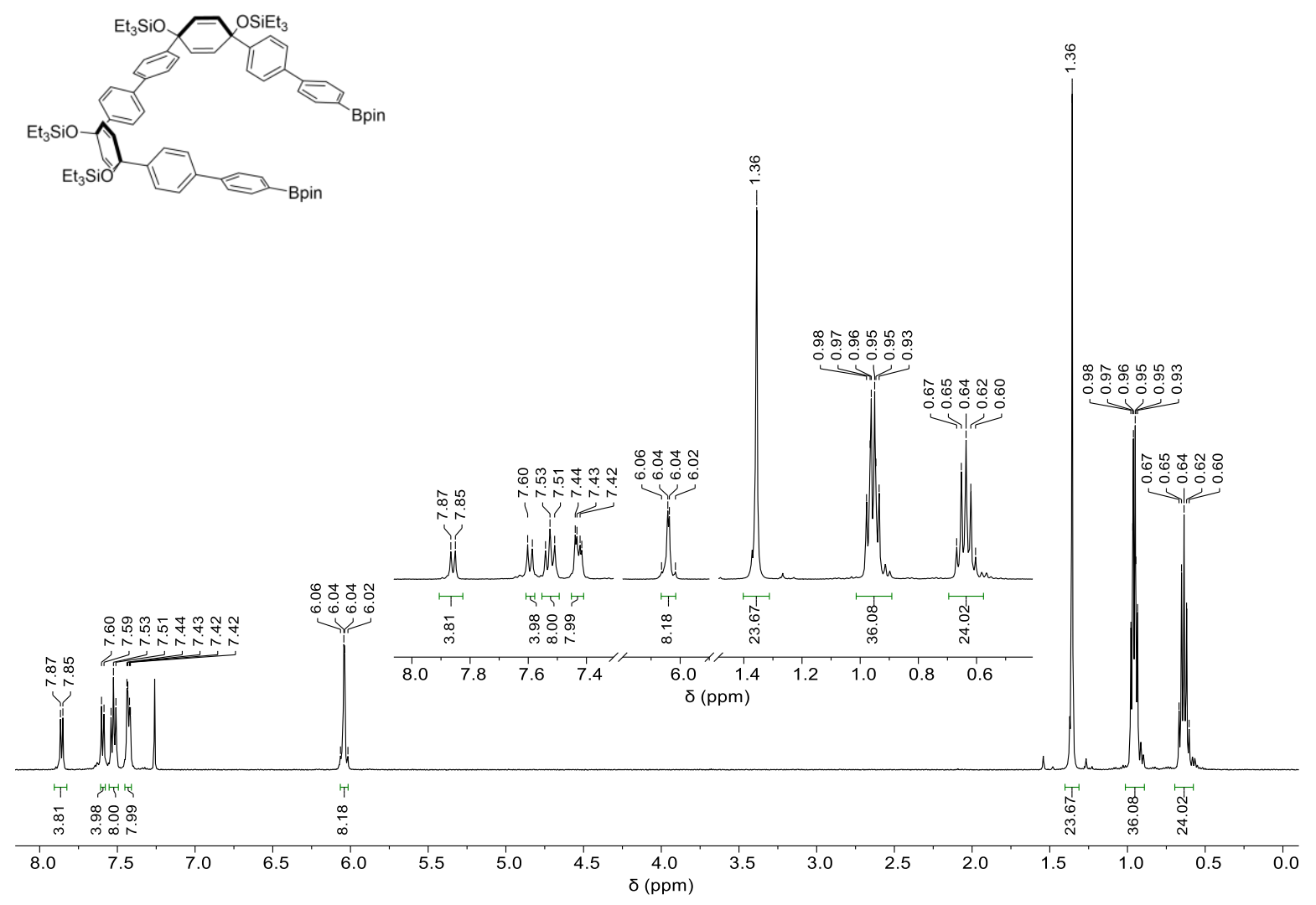

Figure S9: ${ }^{1} \mathrm{H}$ NMR spectrum $(500 \mathrm{MHz})$ of 6 in $\mathrm{CDCl}_{3}$ at room temperature.
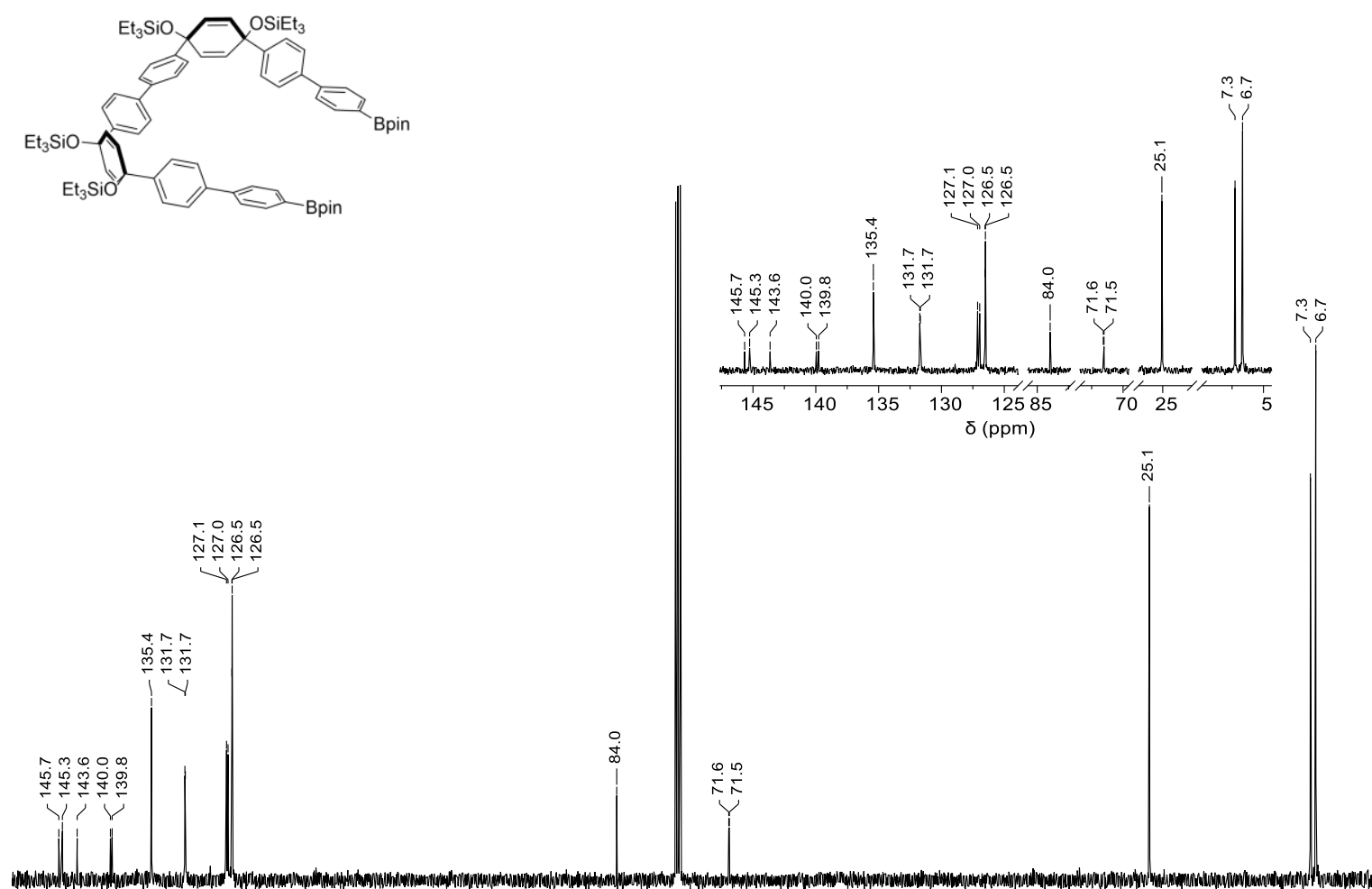

$\begin{array}{llllllllllllllllllllllllllllllllllllllllll}150 & 145 & 140 & 135 & 130 & 125 & 120 & 115 & 110 & 105 & 100 & 95 & 90 & 85 & 80 & 75 & 70 & 65 & 60 & 55 & 50 & 45 & 40 & 35 & 30 & 25 & 20 & 15 & 10 & 5 & 0\end{array}$

Figure S10: ${ }^{13} \mathrm{C}$ NMR spectrum (126 MHz) of 6 in $\mathrm{CDCl}_{3}$ at room temperature. 

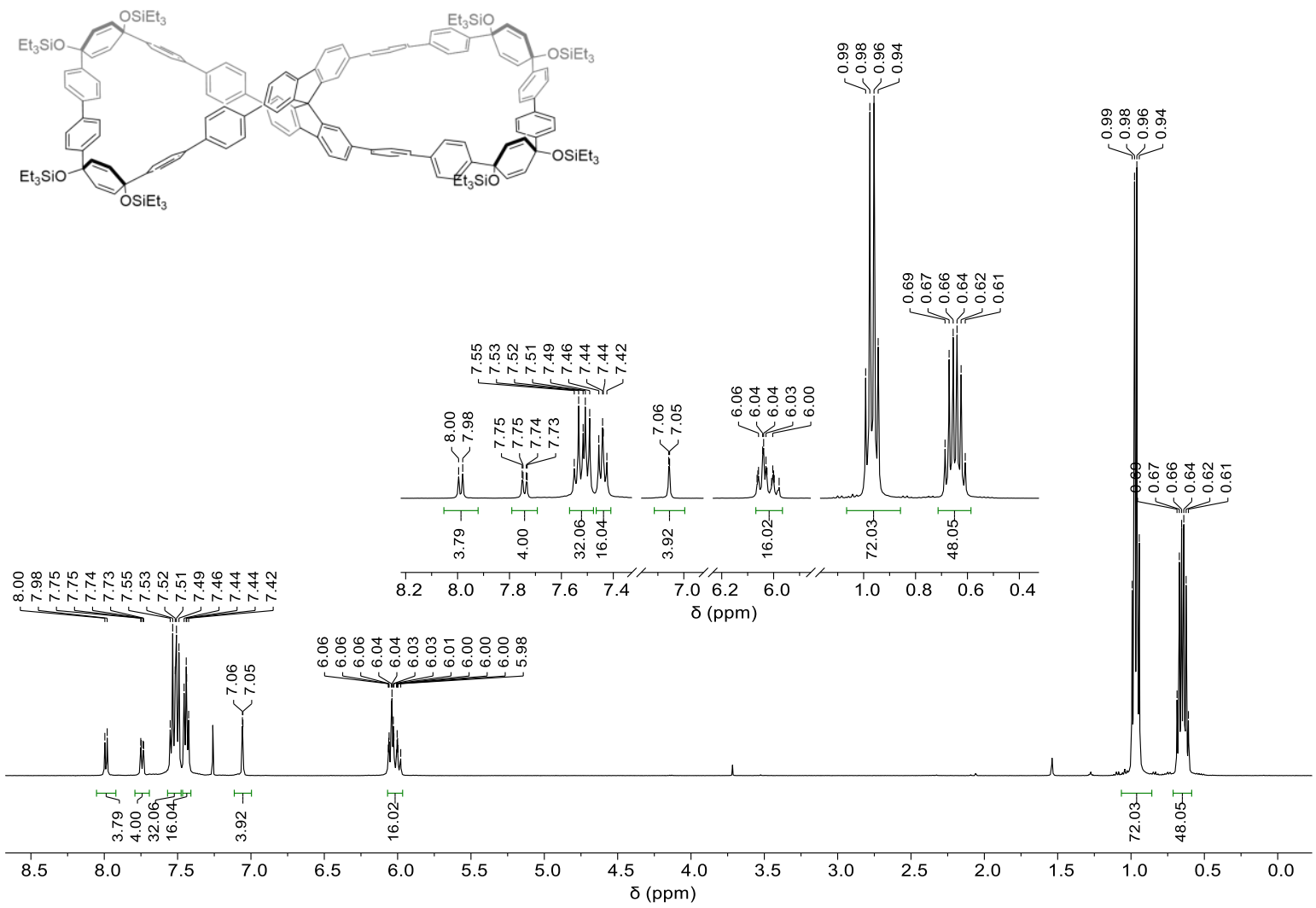

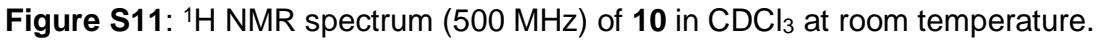
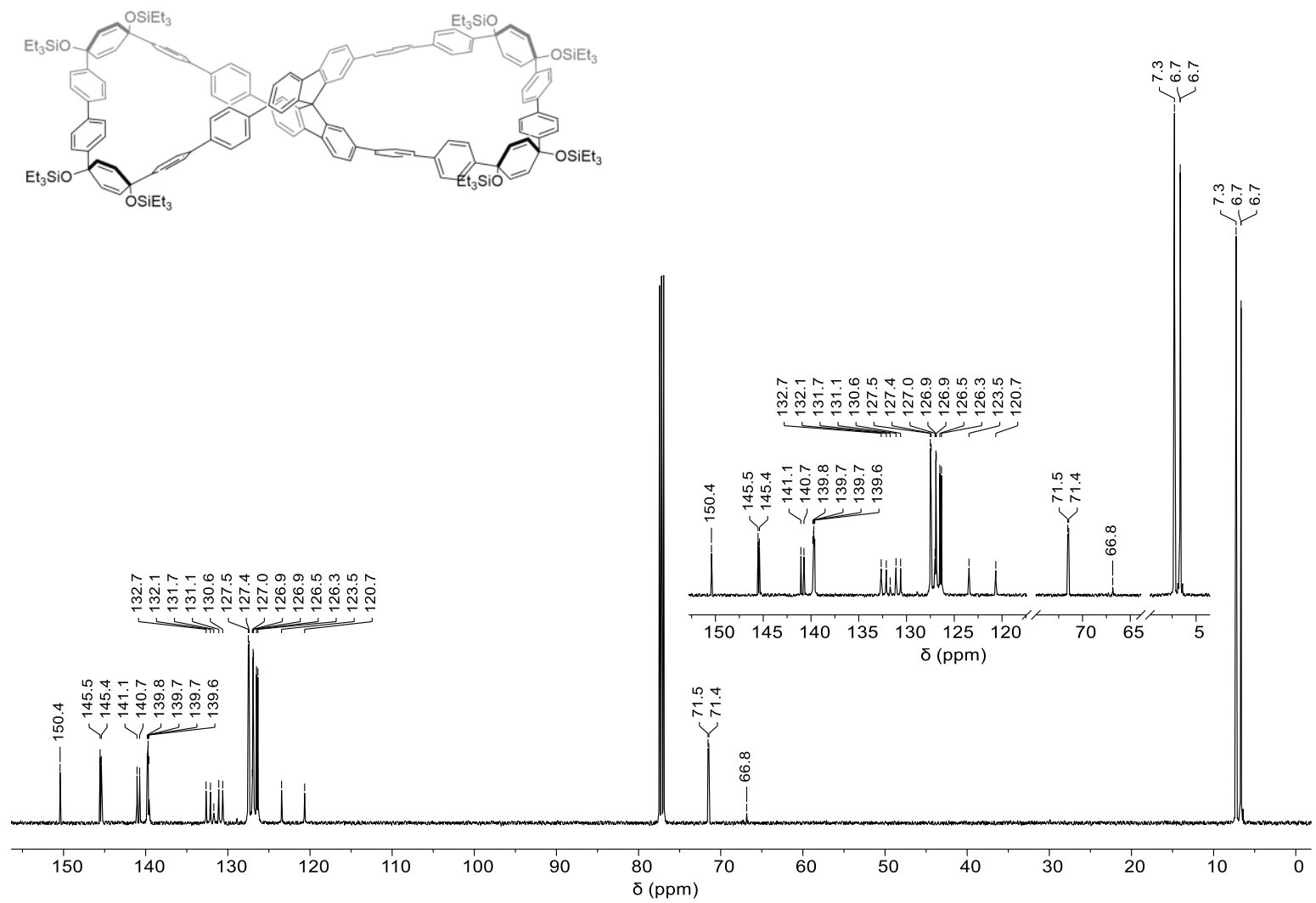

Figure S12: ${ }^{13} \mathrm{C}$ NMR spectrum $(126 \mathrm{MHz})$ of $10 \mathrm{in} \mathrm{CDCl}_{3}$ at room temperature. 


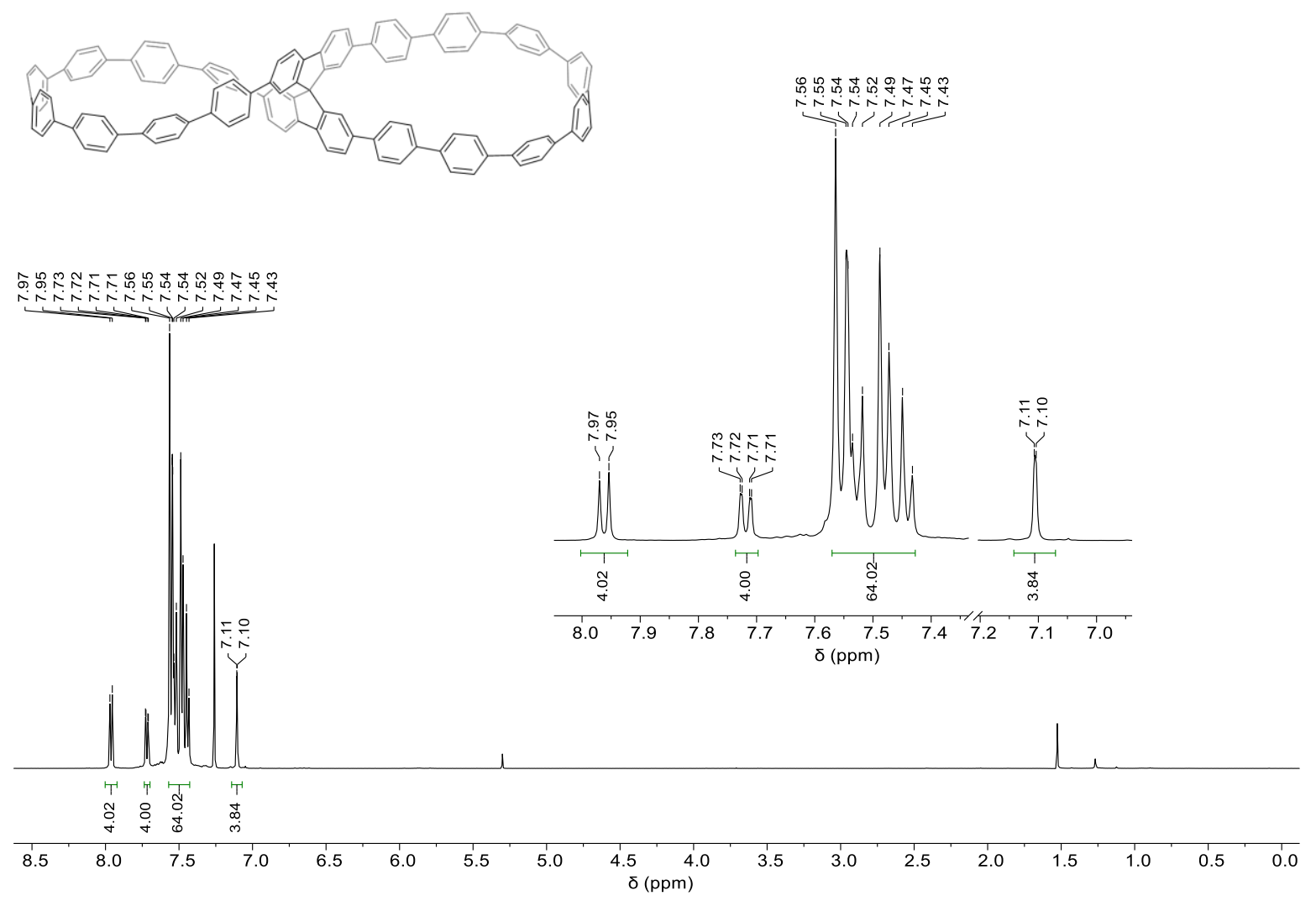

Figure S13: ${ }^{1} \mathrm{H}$ NMR spectrum $(500 \mathrm{MHz})$ of 2 in $\mathrm{CDCl}_{3}$ at room temperature.
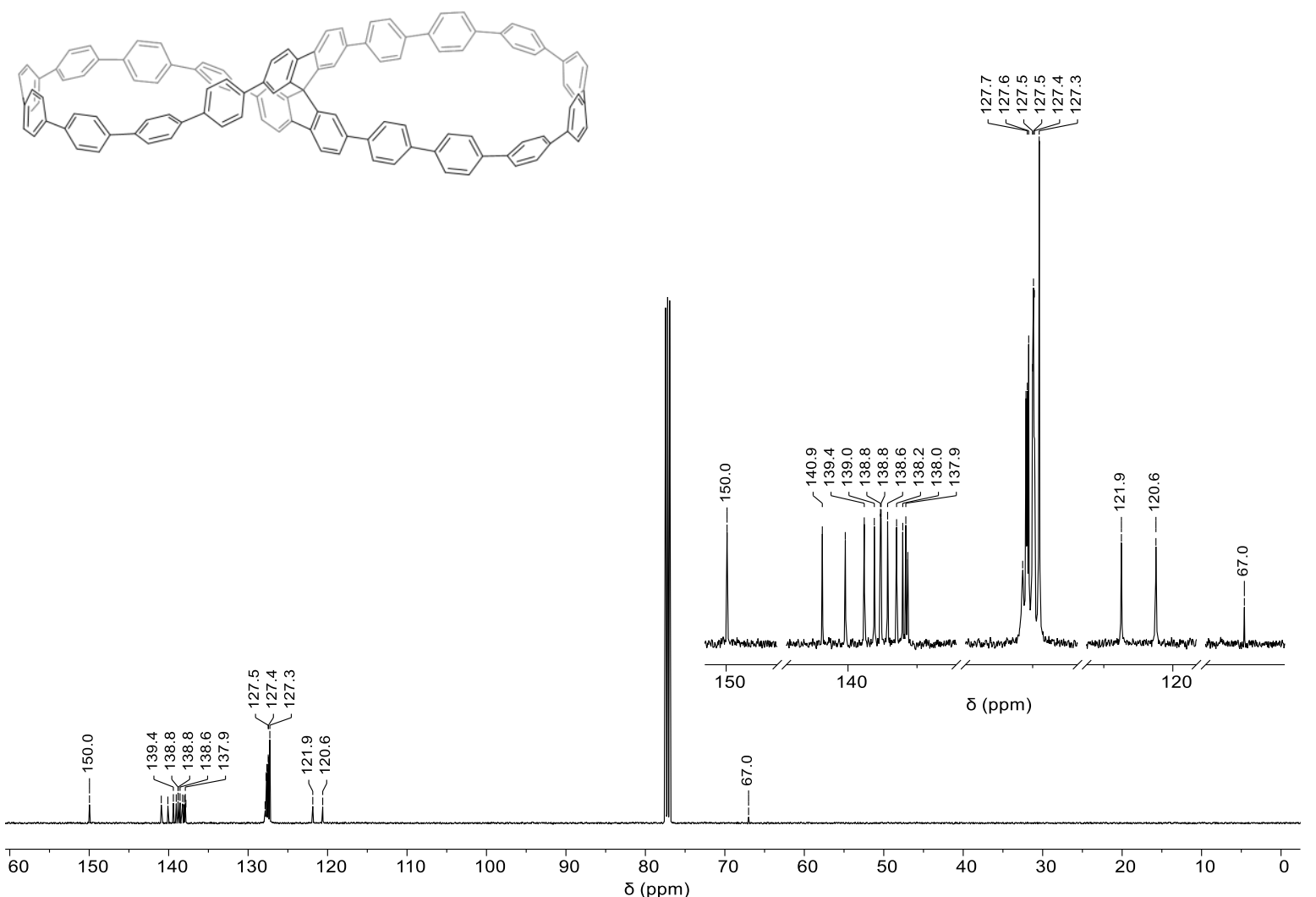

Figure S14: ${ }^{13} \mathrm{C}$ NMR spectrum (126 MHz) of 2 in $\mathrm{CDCl}_{3}$ at room temperature. 

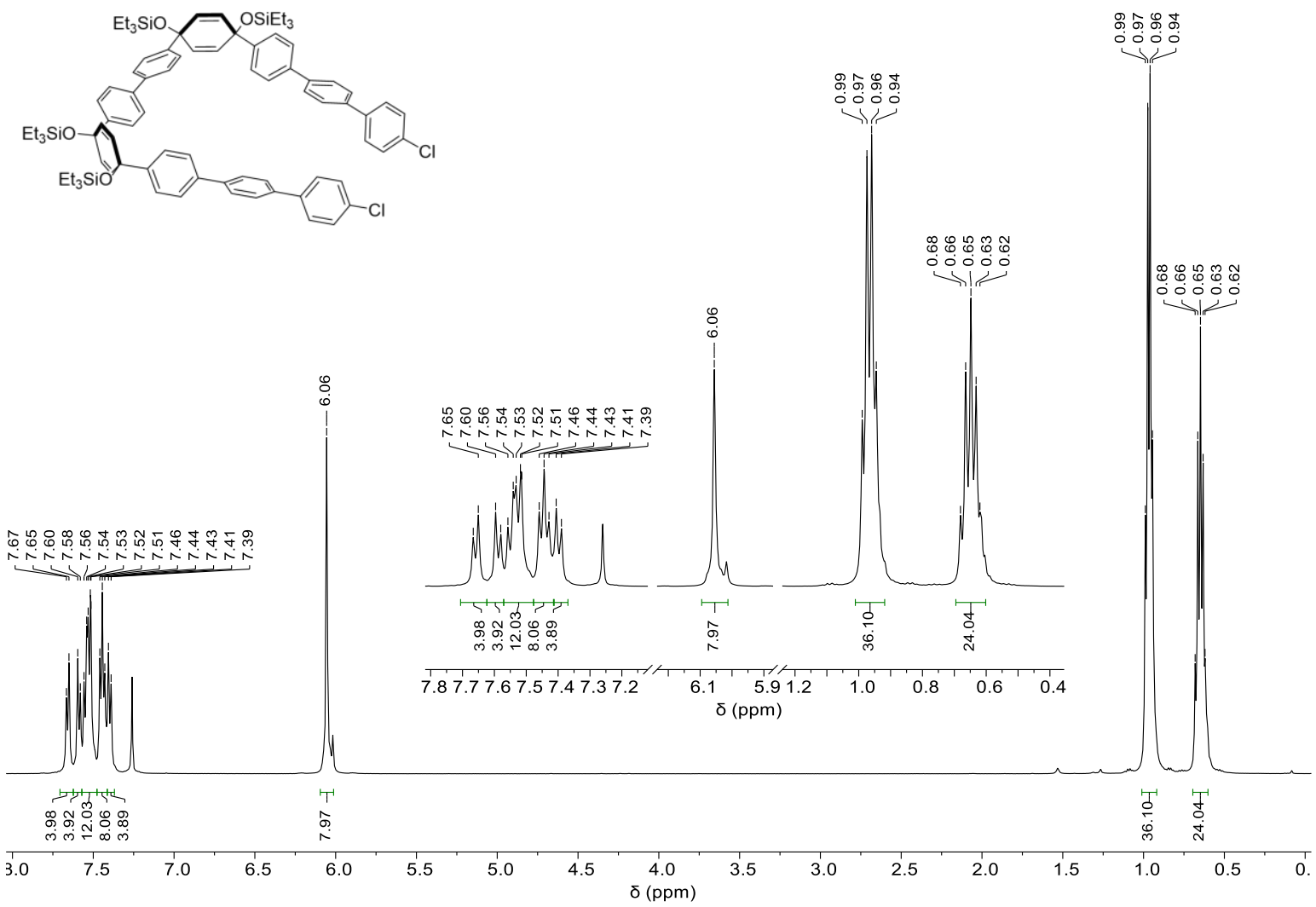

Figure S15: ${ }^{1} \mathrm{H}$ NMR spectrum $(500 \mathrm{MHz})$ of $\mathbf{S} 3$ in $\mathrm{CDCl}_{3}$ at room temperature.
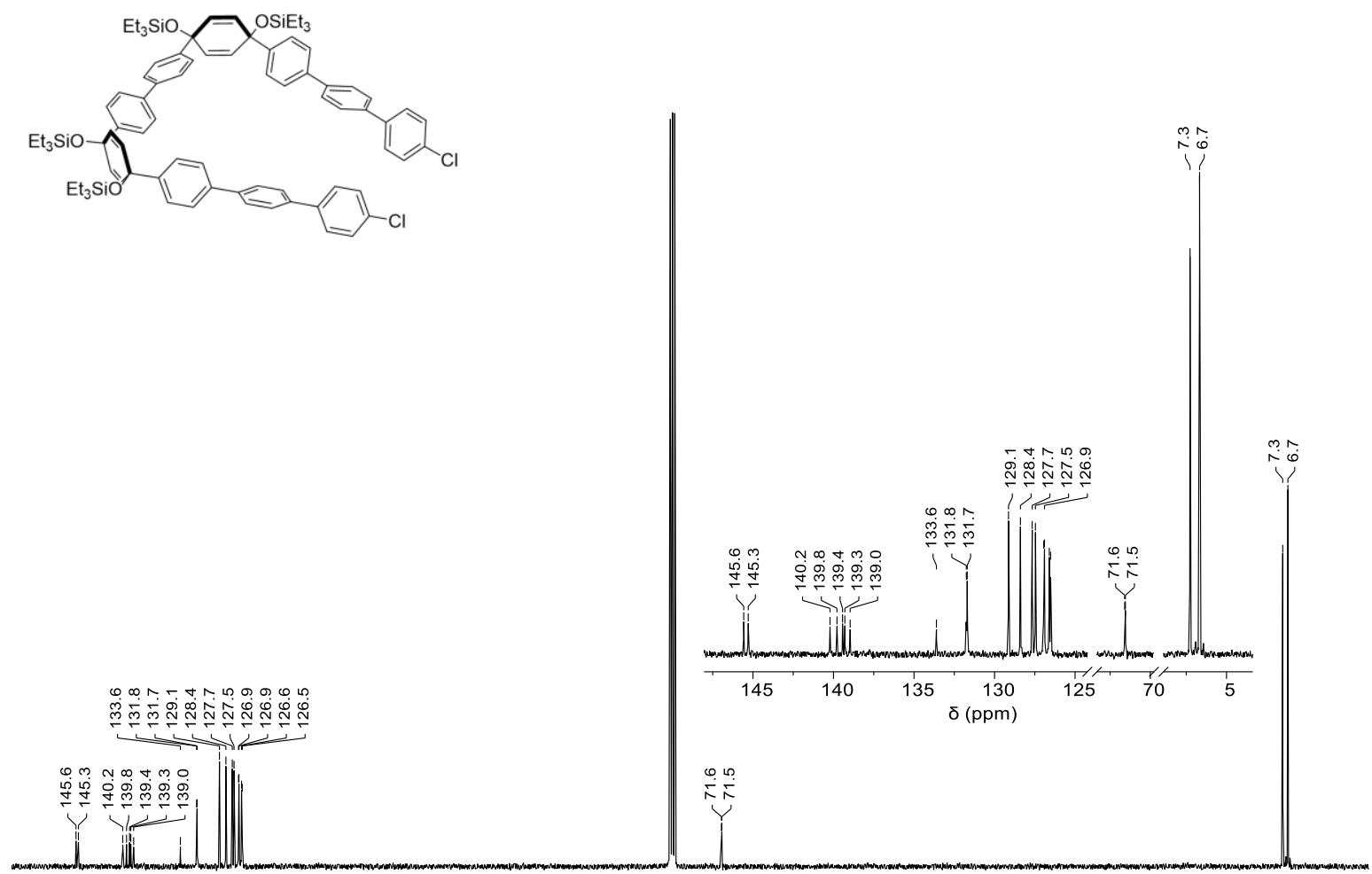

$\begin{array}{lllllllllllllllllllllllllllllllllllllll}150 & 145 & 140 & 135 & 130 & 125 & 120 & 115 & 110 & 105 & 100 & 95 & 90 & 85 & 80 & 75 & 70 & 65 & 60 & 55 & 50 & 45 & 40 & 35 & 30 & 25 & 20 & 15 & 10 & 5 & 0\end{array}$

Figure S16: ${ }^{13} \mathrm{C}$ NMR spectrum $(126 \mathrm{MHz})$ of $\mathbf{S} 3$ in $\mathrm{CDCl}_{3}$ at room temperature. 

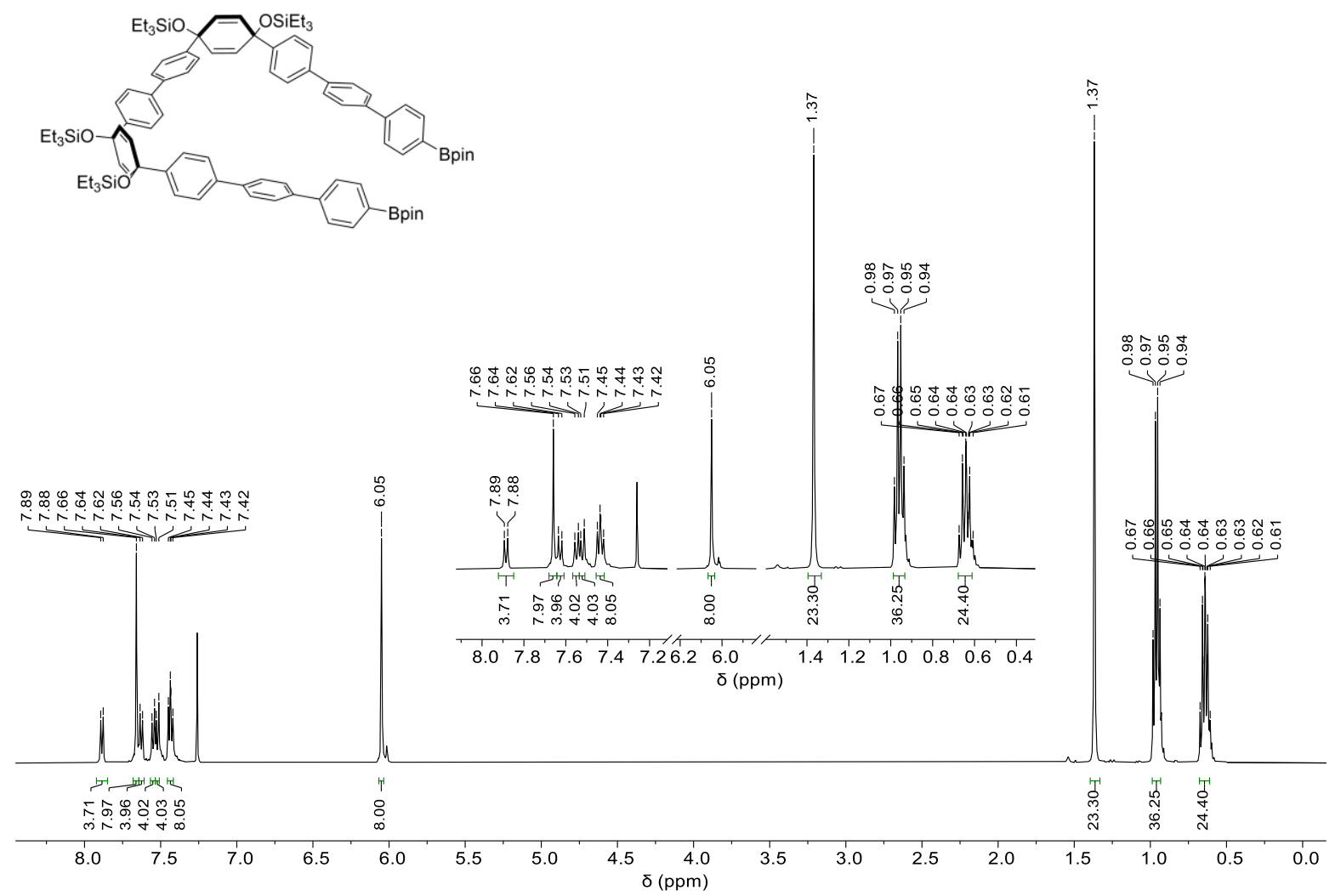

Figure S17: ${ }^{1} \mathrm{H}$ NMR spectrum $(500 \mathrm{MHz})$ of 7 in $\mathrm{CDCl}_{3}$ at room temperature.
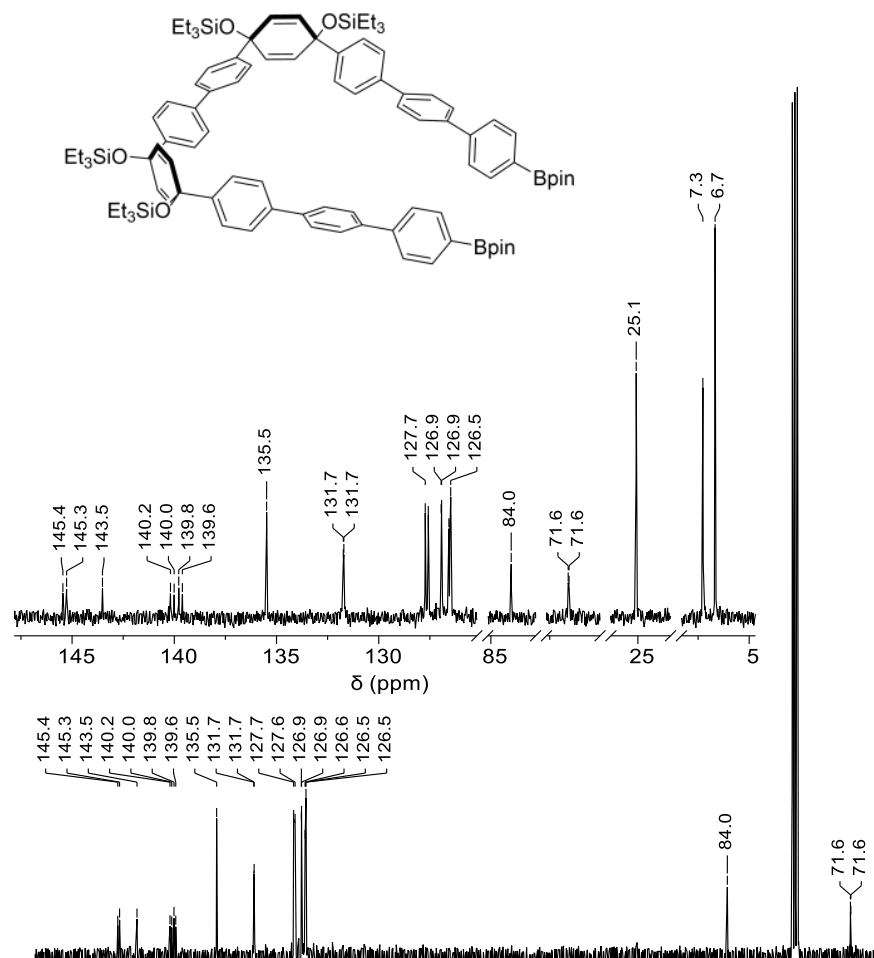

$\begin{array}{llllllllllllllllllllllllllllllllllll}150 & 145 & 140 & 135 & 130 & 125 & 120 & 115 & 110 & 105 & 100 & 95 & 90 & 85 & 80 & 75 & 70 & 65 & 60 & 55 & 50 & 45 & 40 & 35 & 30 & 25 & 20 & 15 & 10 & 5 & 0\end{array}$

Figure S18: ${ }^{13} \mathrm{C}$ NMR spectrum $(126 \mathrm{MHz})$ of 7 in $\mathrm{CDCl}_{3}$ at room temperature. 


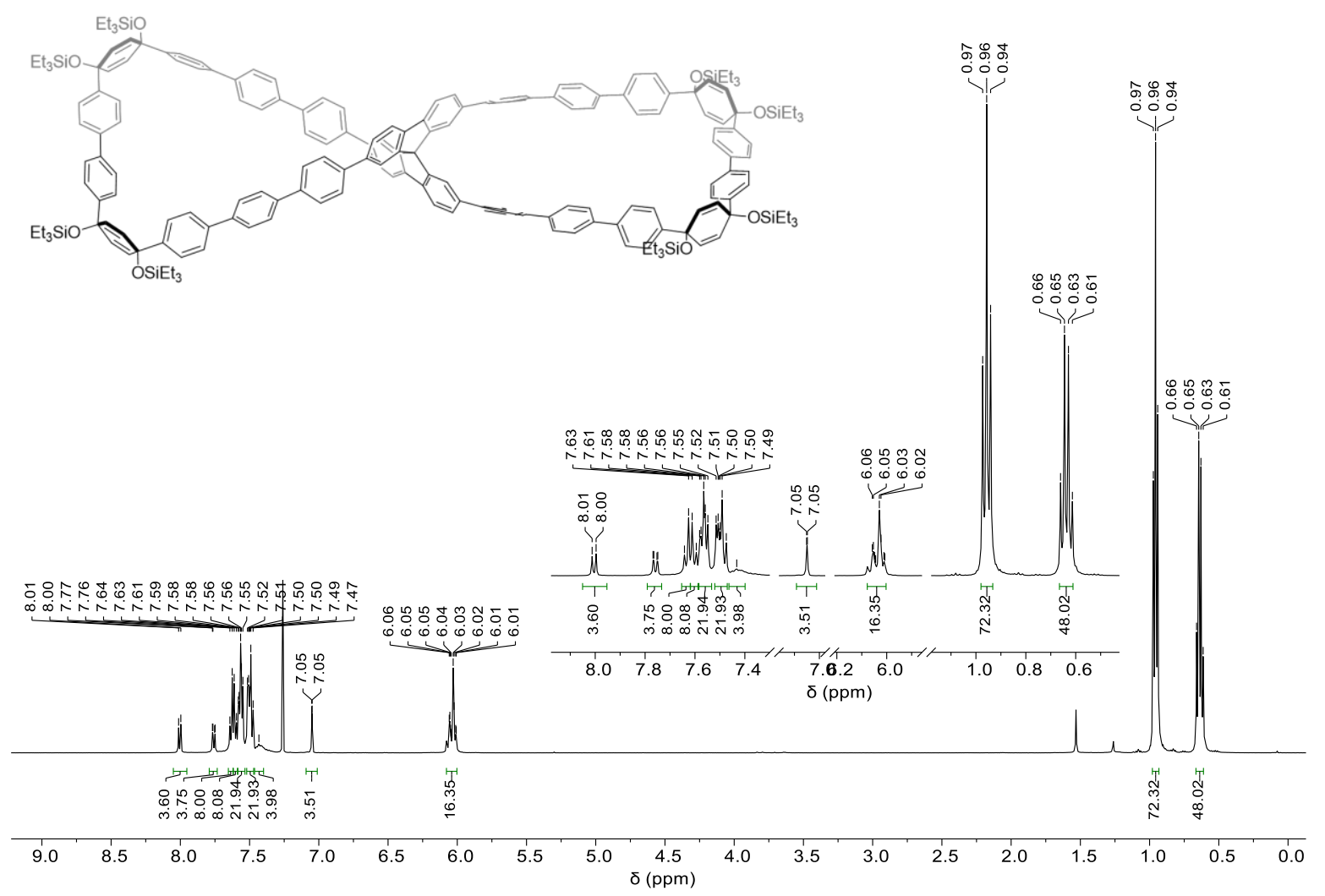

Figure S19: ${ }^{1} \mathrm{H}$ NMR spectrum $(500 \mathrm{MHz})$ of 11 in $\mathrm{CDCl}_{3}$ at room temperature.
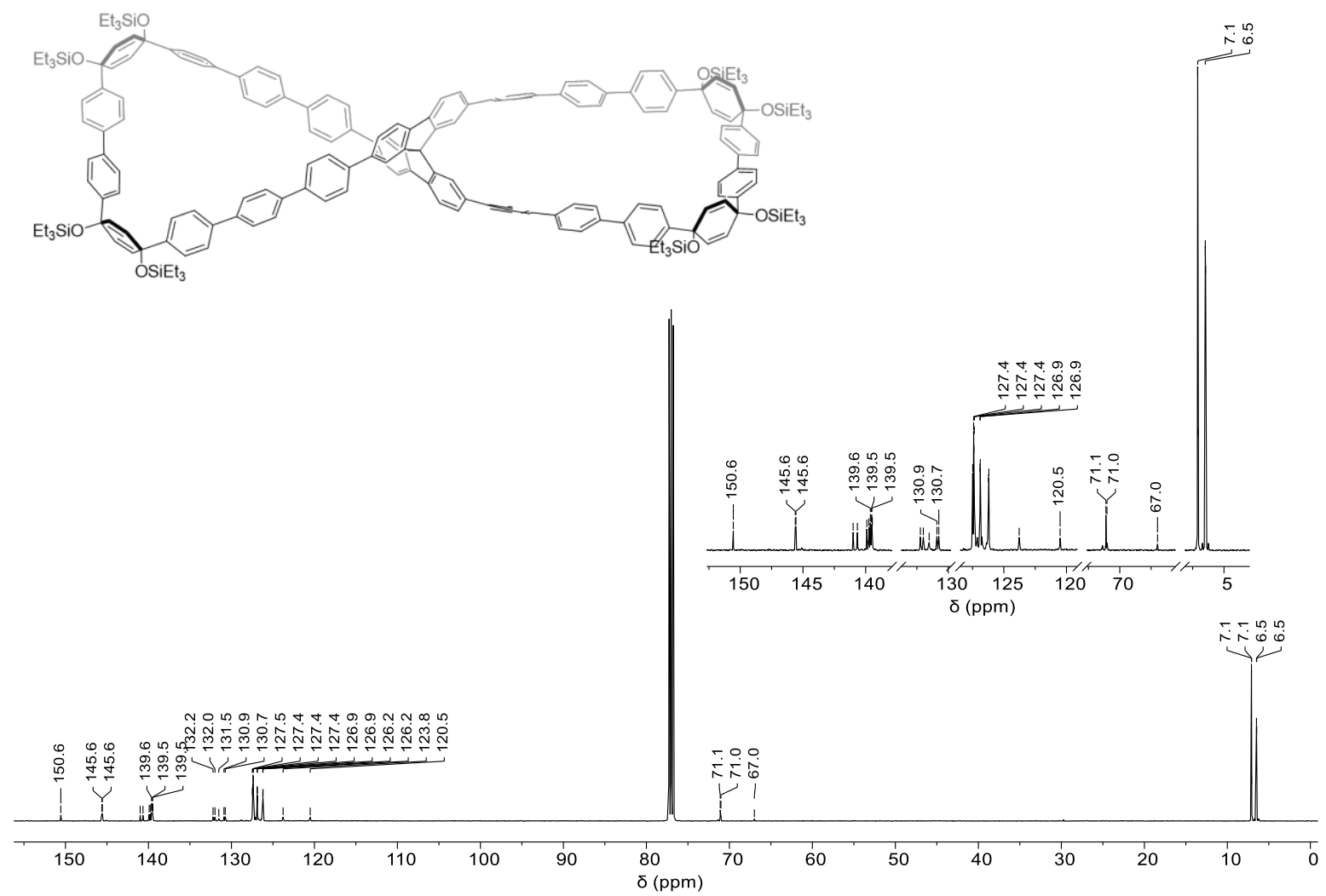

Figure S20: ${ }^{13} \mathrm{C}$ NMR spectrum (126 MHz) of 11 in $\mathrm{CDCl}_{3}$ at room temperature. 


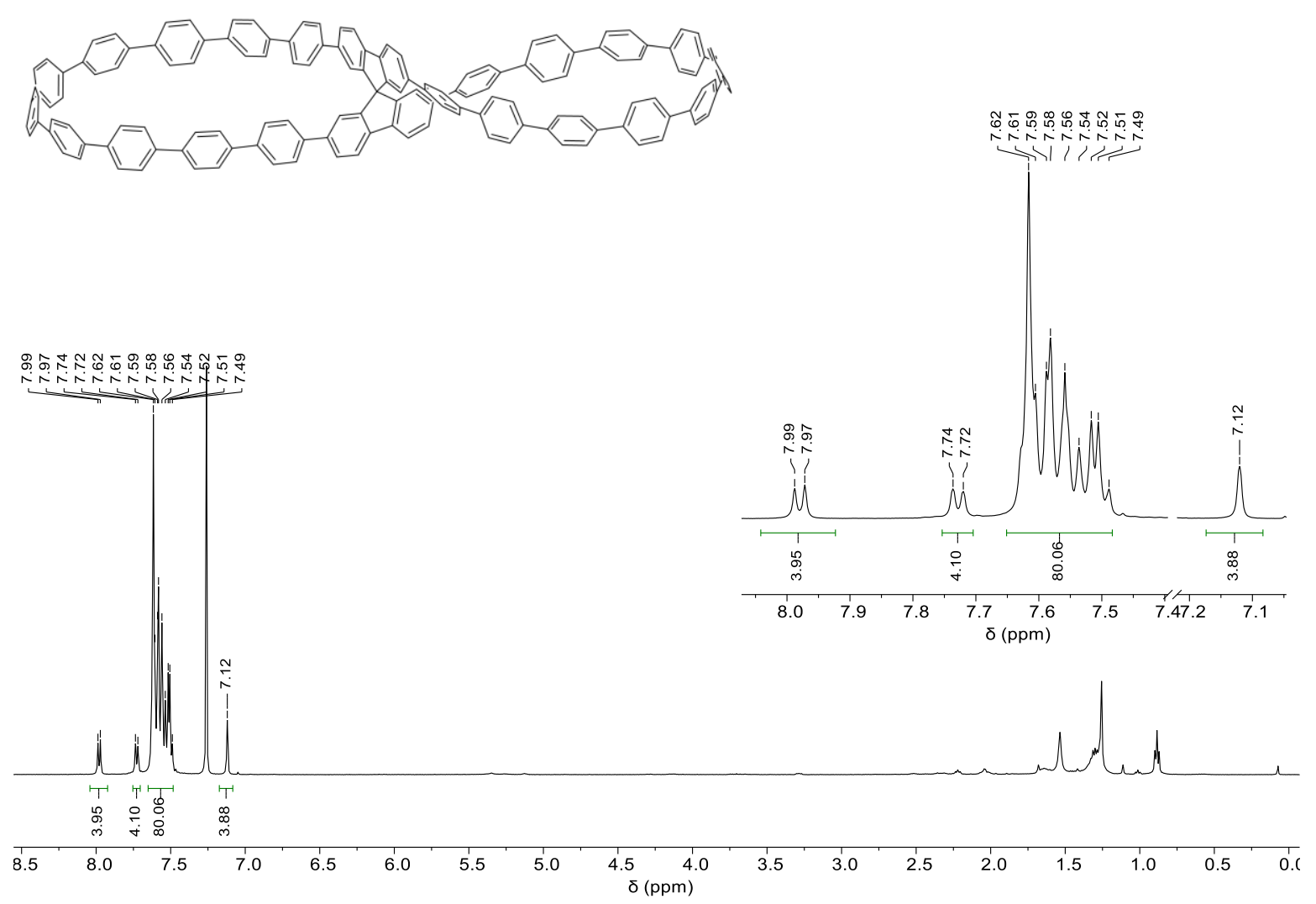

Figure S21: ${ }^{1} \mathrm{H}$ NMR spectrum $(500 \mathrm{MHz})$ of $\mathbf{3}$ in $\mathrm{CDCl}_{3}$ at room temperature.
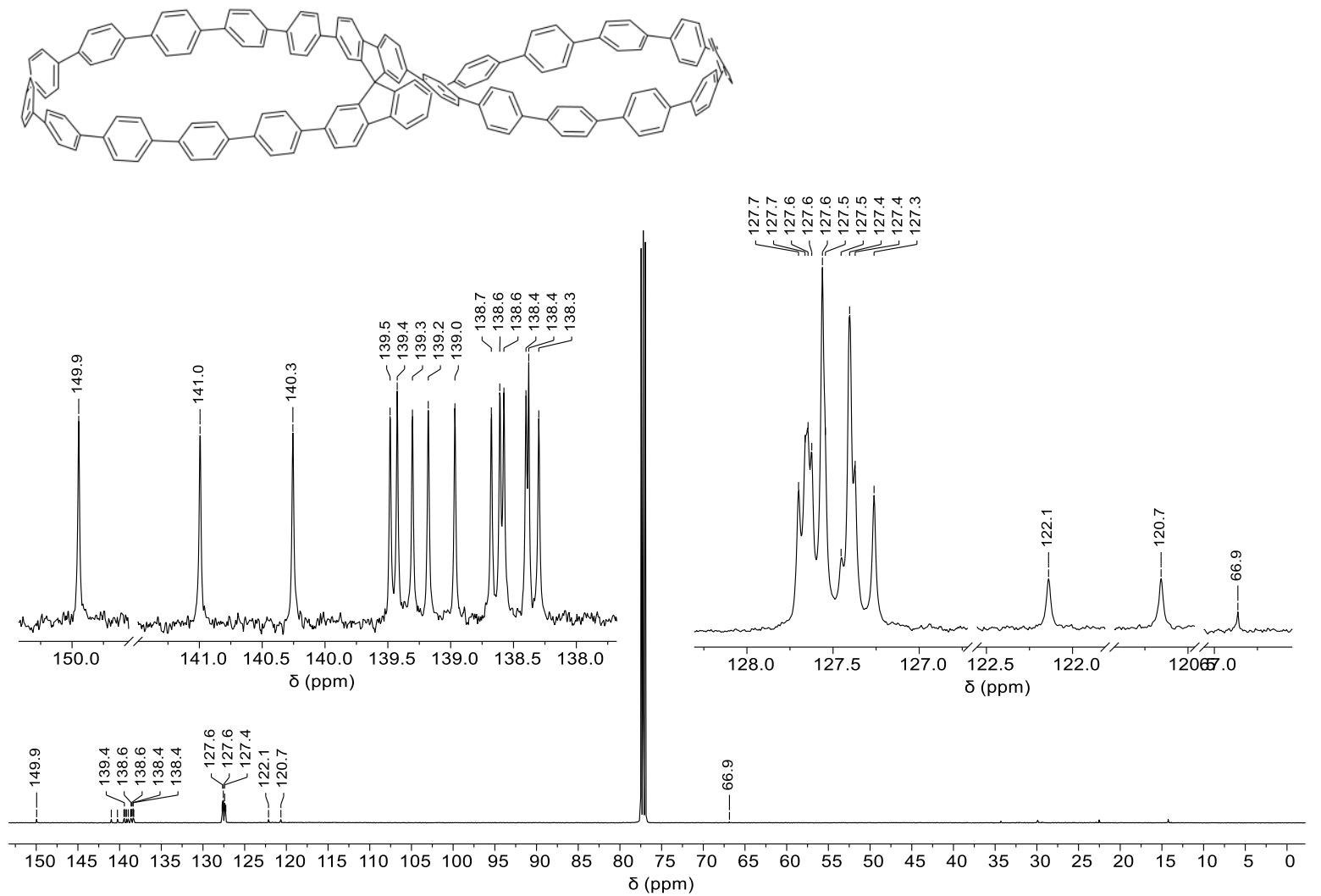

Figure S22: ${ }^{13} \mathrm{C}$ NMR spectrum ( $\left.126 \mathrm{MHz}\right)$ of 3 in $\mathrm{CDCl}_{3}$ at room temperature. 

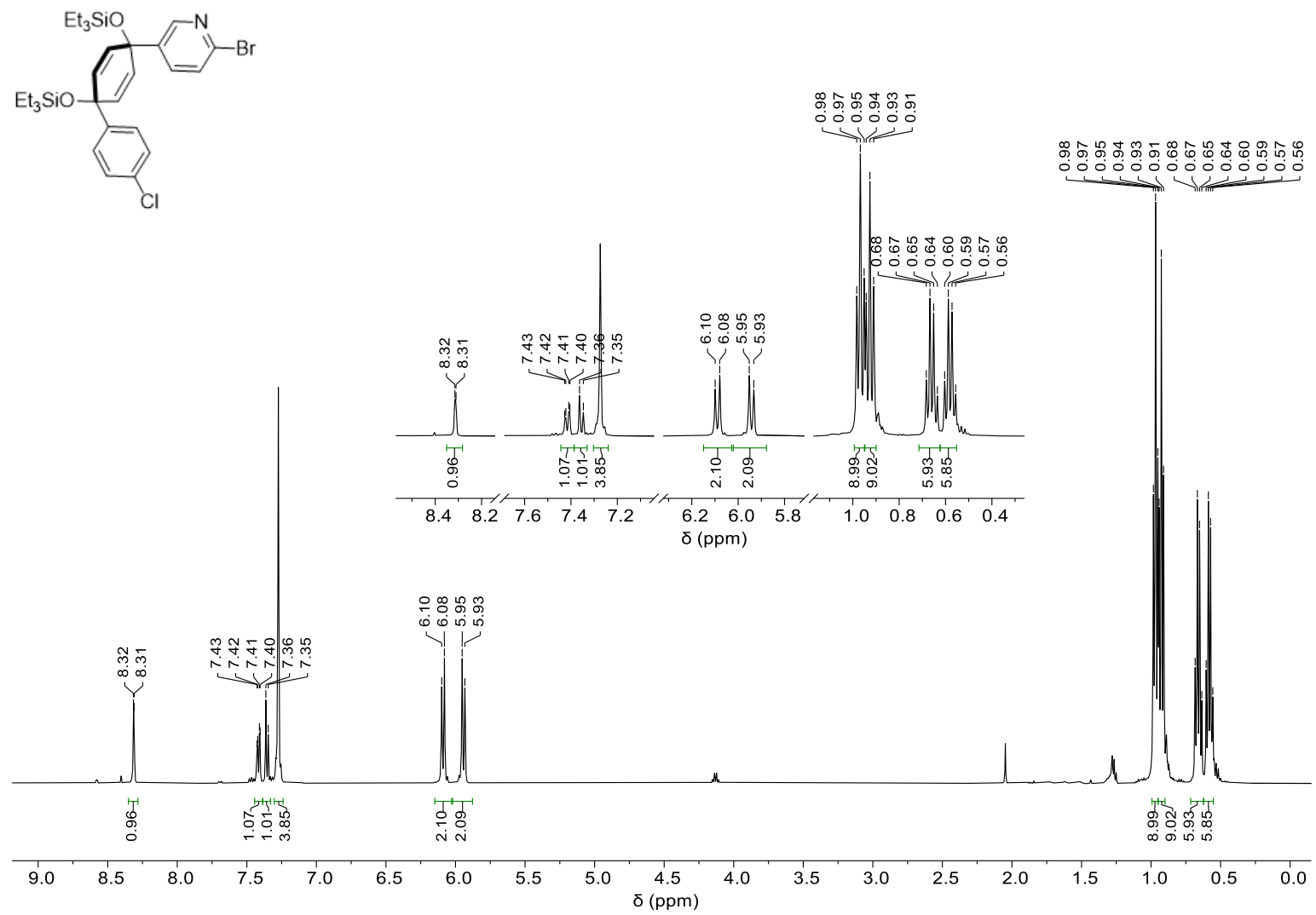

Figure S23: ${ }^{1} \mathrm{H}$ NMR spectrum $(500 \mathrm{MHz})$ of $\mathbf{S} 5$ in $\mathrm{CDCl}_{3}$ at room temperature.
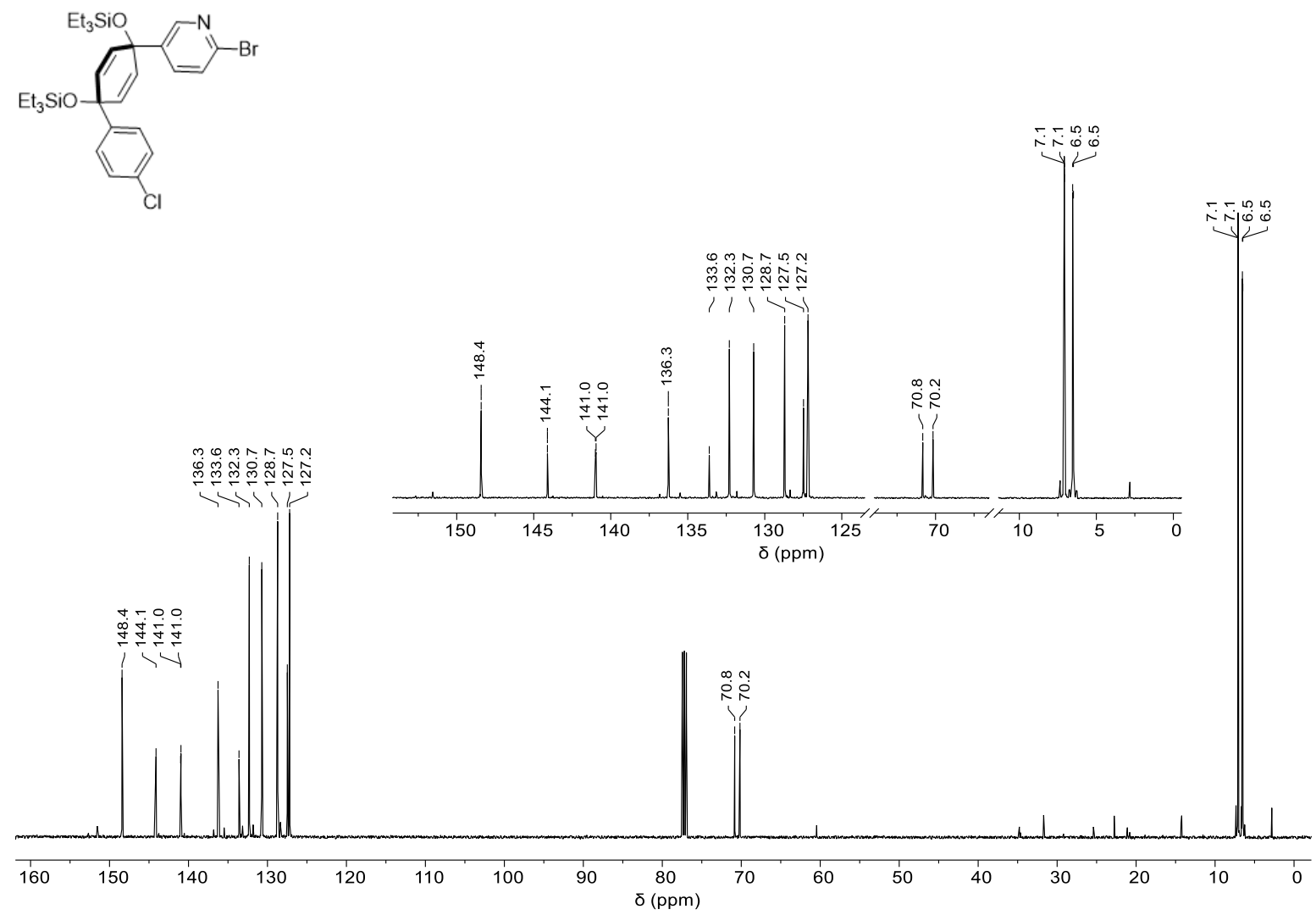

Figure S24: ${ }^{13} \mathrm{C}$ NMR spectrum $(126 \mathrm{MHz})$ of $\mathbf{S} 5$ in $\mathrm{CDCl}_{3}$ at room temperature. 

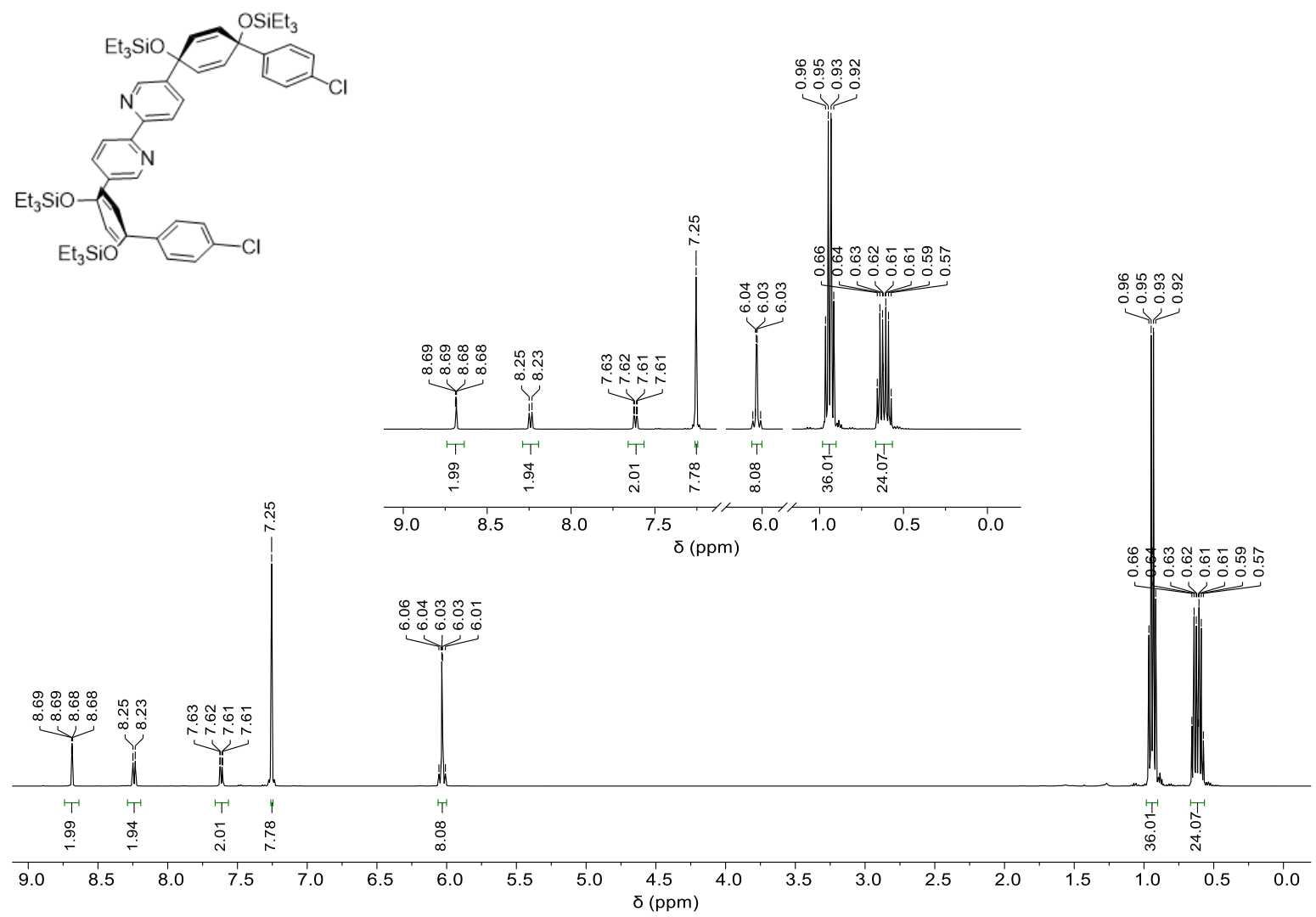

Figure S25: ${ }^{1} \mathrm{H}$ NMR spectrum $(500 \mathrm{MHz})$ of $\mathbf{S} 6$ in $\mathrm{CDCl}_{3}$ at room temperature.
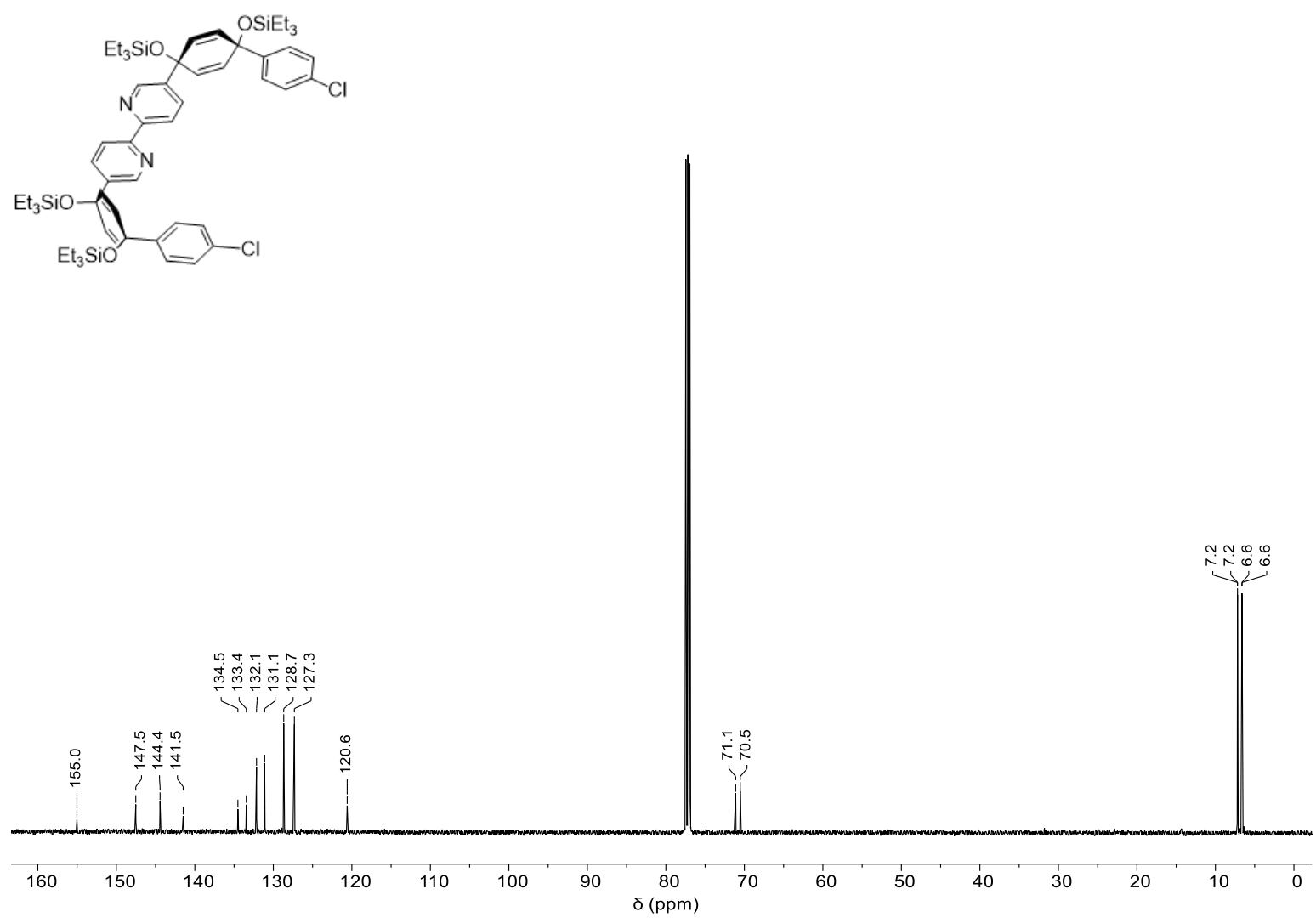

Figure S26: ${ }^{13} \mathrm{C}$ NMR spectrum $(126 \mathrm{MHz})$ of $\mathbf{S} 6$ in $\mathrm{CDCl}_{3}$ at room temperature. 

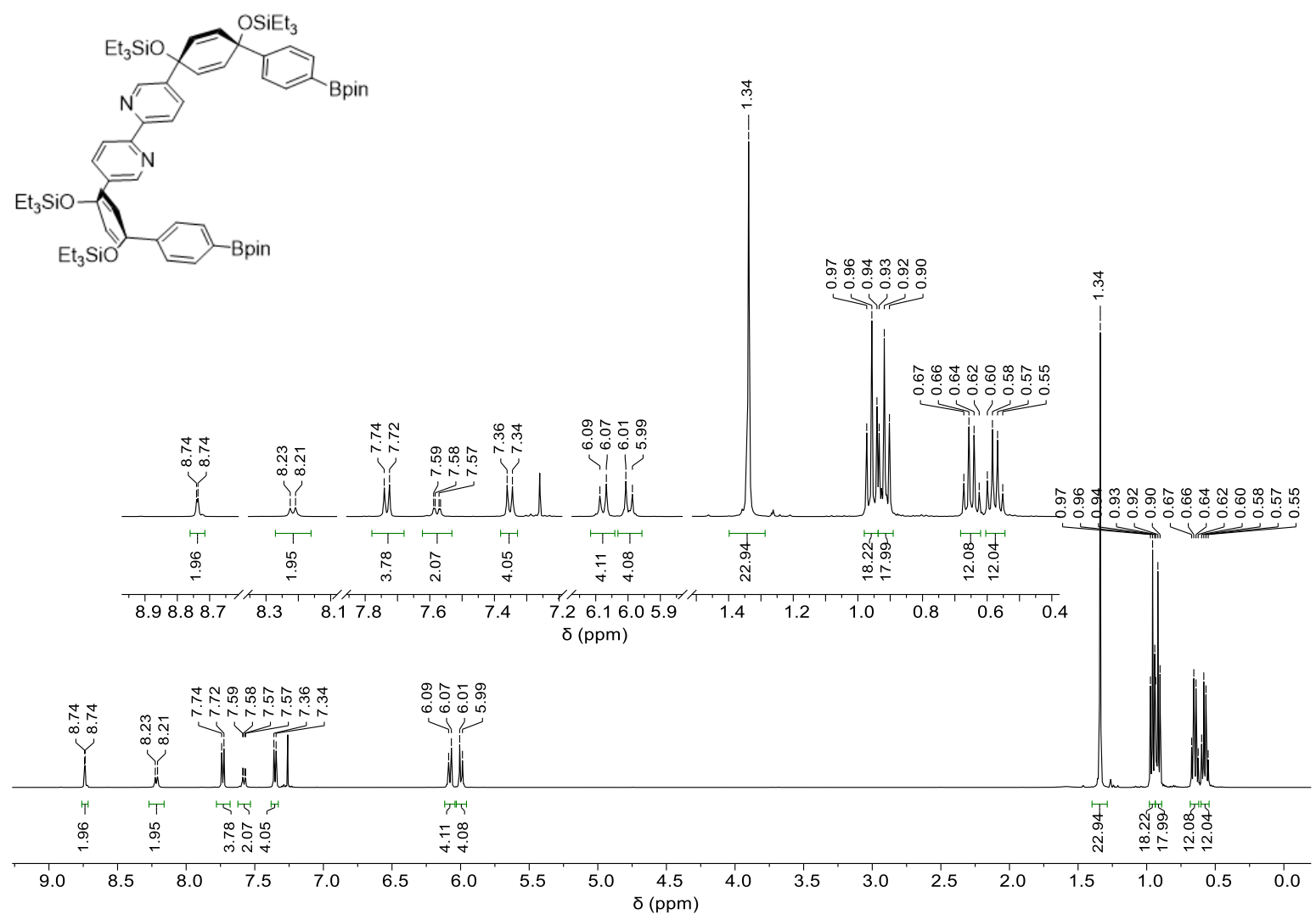

Figure S27: ${ }^{1} \mathrm{H}$ NMR spectrum $(500 \mathrm{MHz})$ of 8 in $\mathrm{CDCl}_{3}$ at room temperature.
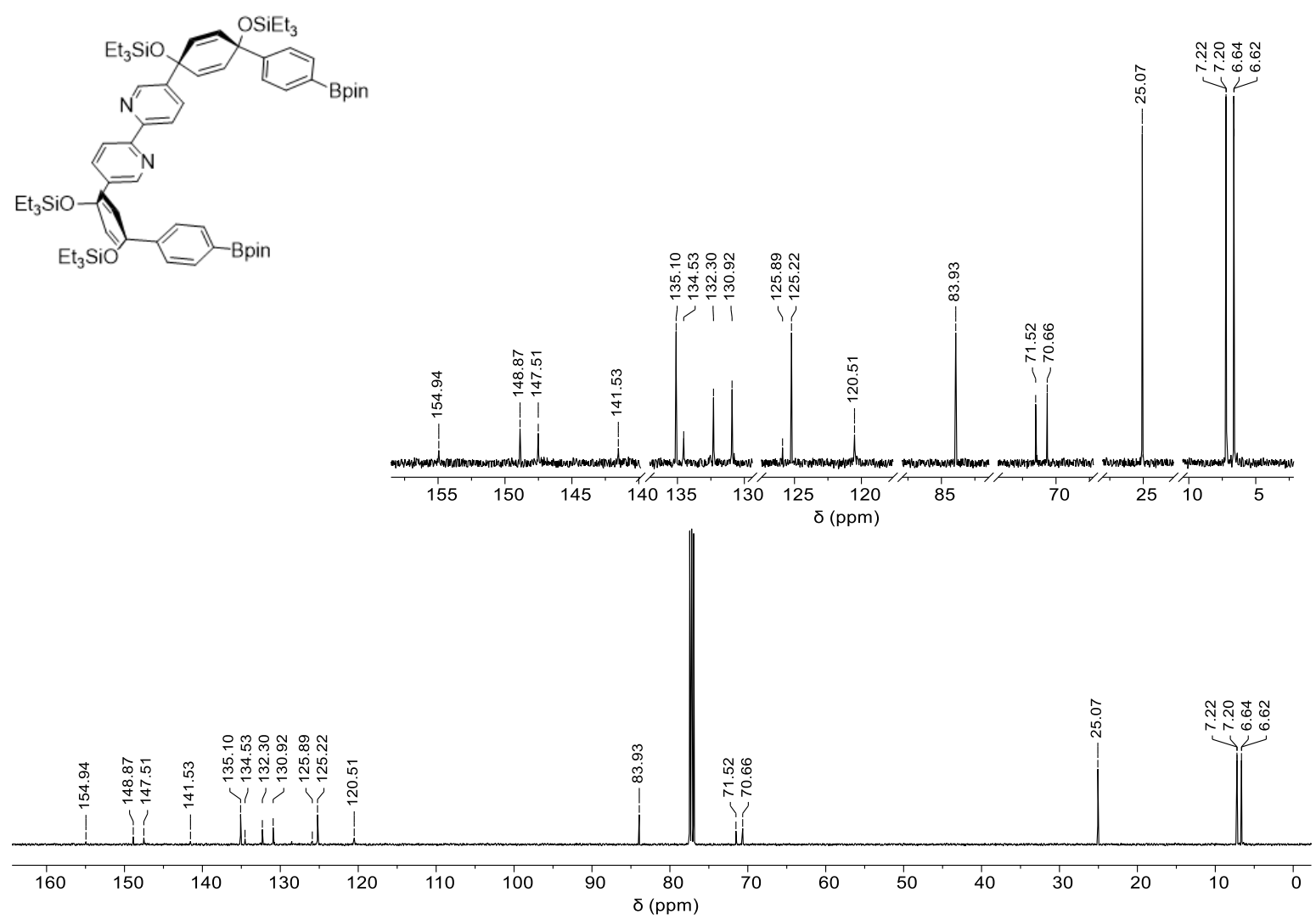

Figure S28: ${ }^{13} \mathrm{C}$ NMR spectrum $(126 \mathrm{MHz})$ of 8 in $\mathrm{CDCl}_{3}$ at room temperature. 


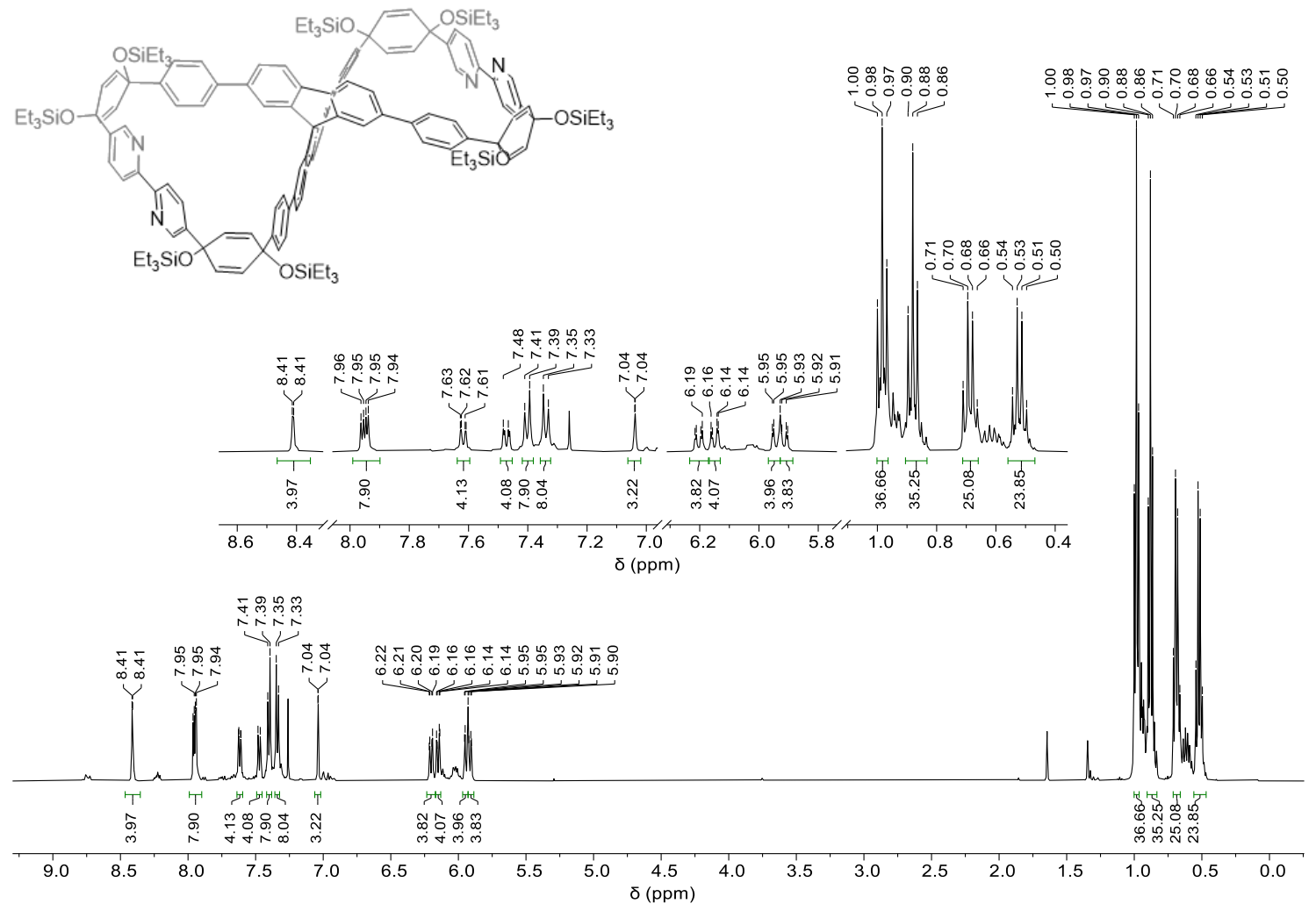

Figure S29: ${ }^{1} \mathrm{H}$ NMR spectrum $(500 \mathrm{MHz})$ of 12 in $\mathrm{CDCl}_{3}$ at room temperature.
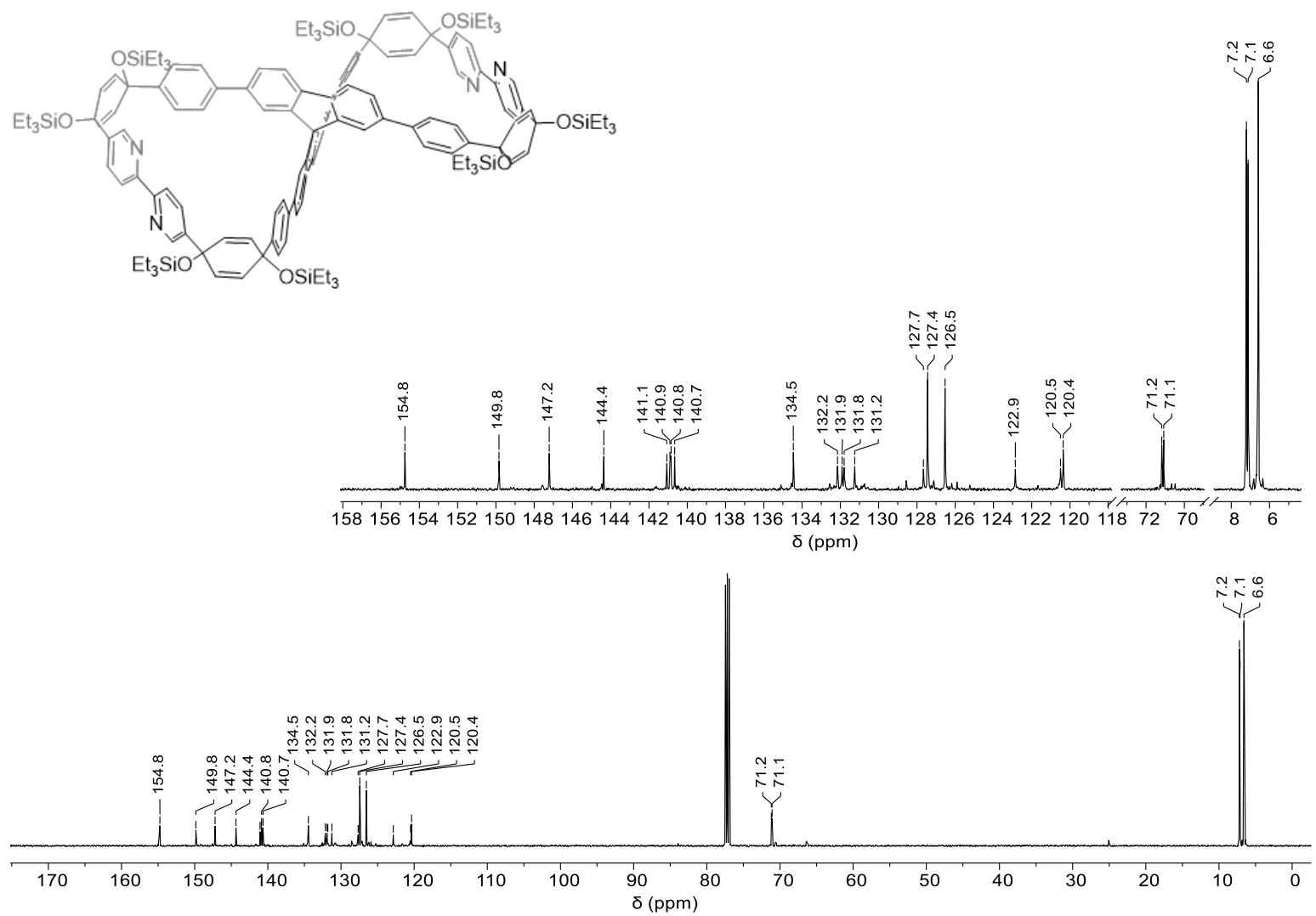

Figure S30: ${ }^{13} \mathrm{C}$ NMR spectrum (126 MHz) of 12 in $\mathrm{CDCl}_{3}$ at room temperature. 

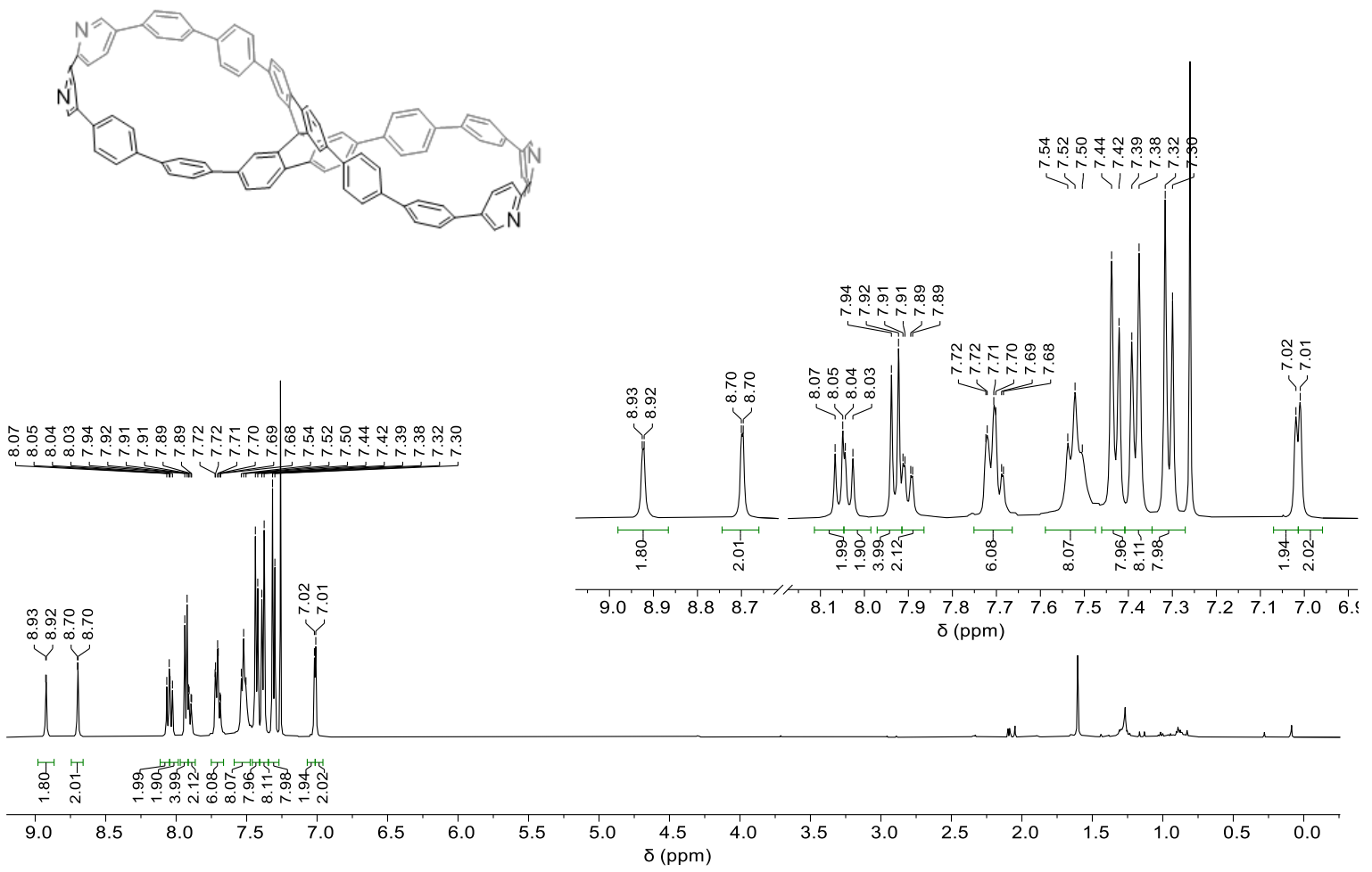

Figure S31: ${ }^{1} \mathrm{H}$ NMR spectrum $(500 \mathrm{MHz})$ of 4 in $\mathrm{CDCl}_{3}$ at room temperature.
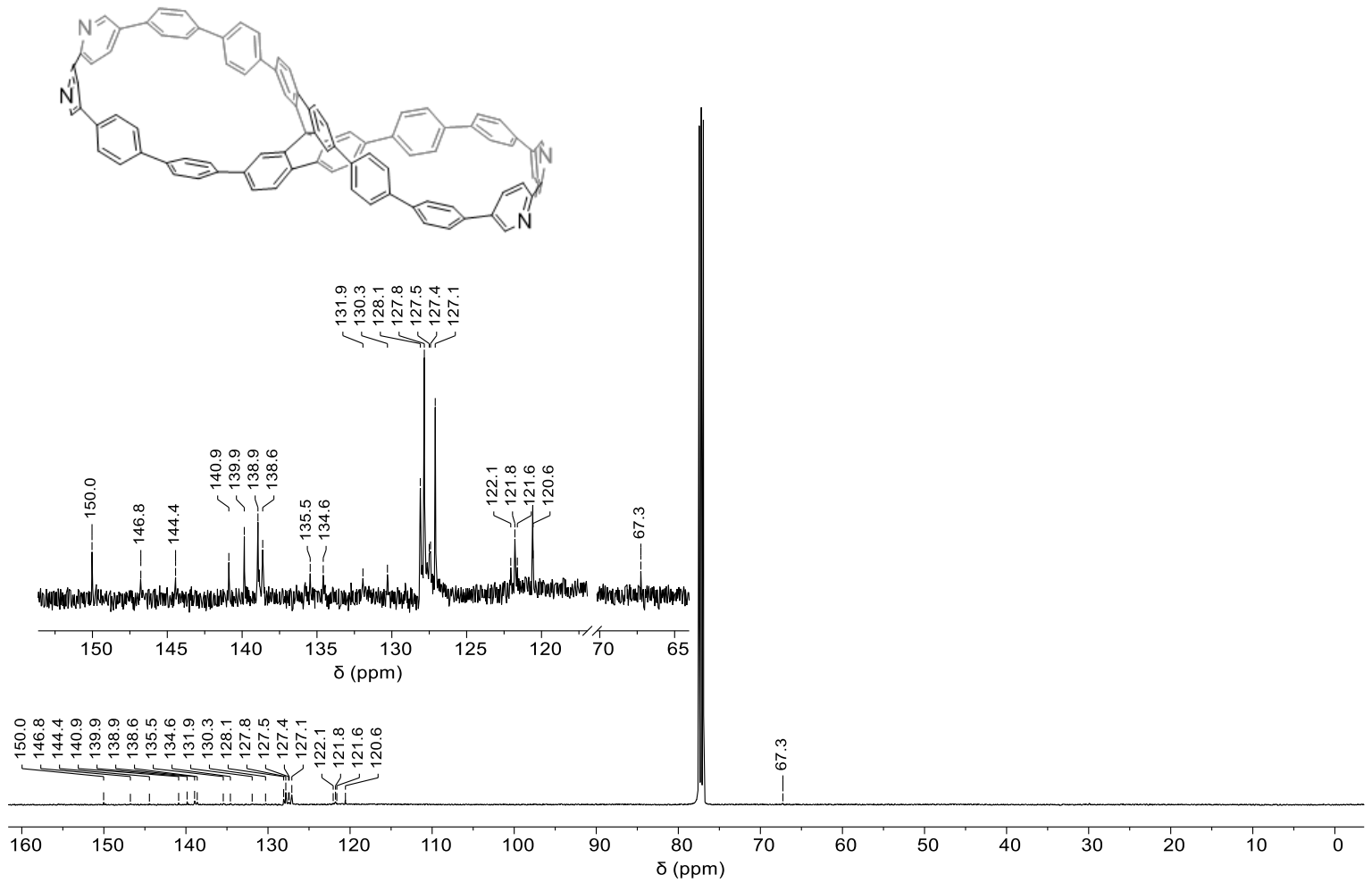

Figure S32: ${ }^{13} \mathrm{C}$ NMR spectrum (126 MHz) of 4 in $\mathrm{CDCl}_{3}$ at room temperature. 


\section{X-Ray Crystallographic Analysis}

Single crystals suitable for crystallographic analysis were grown from slow diffusion of pentane into a solution 1 in $\mathrm{CHCl}_{3}$ at room temperature over 2 days, from slow diffusion of pentane into a solution 2 in $\mathrm{CH}_{2} \mathrm{Cl}_{2}$ at room temperature over 3 days, and from slow vapor diffusion of pentane into a solution of 4 in THF at $7{ }^{\circ} \mathrm{C}$ over $3 \mathrm{~d}$, respectively. Diffraction intensities for all compounds were collected at $173 \mathrm{~K}$ on a Bruker Apex $2 \mathrm{CCD}$ diffractometer using $\mathrm{Cu}-\mathrm{K}_{\alpha}$ radiation, $\lambda=1.54178$ $\AA$. Space groups were determined based on systematic absences for 2 and $\mathbf{4}$ and intensity statistics for compound 1. Absorption corrections were applied by SADABS. ${ }^{[4]}$ Structures were solved by direct methods and Fourier techniques and refined on $F^{2}$ using full matrix least-squares procedures. All non- $\mathrm{H}$ atoms were refined with anisotropic thermal parameters. $\mathrm{H}$ atoms in all structures were refined in calculated positions in a rigid group model. For all investigated structures in this paper X-ray diffraction at high angles is very weak due to presence of numerous disordered solvent molecules in the structures. For compound $\mathbf{2}$ it was only possible to collect data up to $2 \theta_{\max }=99.70^{\circ}$ even using a strong Incoatec $\mathrm{I} \mu \mathrm{S}$ Cu source. However, the diffraction data collected for 2 provide an appropriate number of measured reflections per number of refined parameters, 10263/1171. The resolution for 2 is relatively low due to a lot of disordered fragments in the structures and weak X-ray diffraction at high angles, but the collected data unambiguously proofs the structure of bismacrocyclic nanoloop 2. In all three investigated structures there are a lot of solvent molecules which fill out space in the hoops and between them in the packing. Some of them were located and refined; $4.83 \times \mathrm{CHCl}_{3}$ molecules in $1,3 \times \mathrm{CH}_{2} \mathrm{Cl}_{2}$ molecules in 2 and 5 $\times \mathrm{C}_{4} \mathrm{H}_{8} \mathrm{O}$ molecules in 4 . During refinement some positions of the $\mathrm{CHCl}_{3}$ solvent molecules in $\mathbf{1}$ were found to be not fully occupied. Other solvent molecules in these structures are highly disordered and were treated by SQUEEZE..$^{[5]}$ The corrections of the X-ray data by SQUEEZE are 704 (1), 276 (2) and 936 (4) electrons/cell; the required values are 696, 252 and 800 electron/cell, respectively for $12 \times \mathrm{CHCl}_{3}$ molecules in the full unit cell in $\mathbf{1}$, a mixture of $\mathrm{CH}_{2} \mathrm{Cl}_{2}$ and $\mathrm{C}_{5} \mathrm{H}_{12}$ solvent molecules in six positions in 2 and $20 \mathrm{THF}$ solvent molecules in the full unit cells in $\mathbf{4}$. In case of 2 highly disordered solvent molecules which were not recognized based on the X-ray data were not included in the total formula of the compound. In all structures, thermal parameters for atoms in the located and refined solvent molecules are large and its positions are not precisely determined. The solvent THF molecules in $\mathbf{4}$ were refined under the assumption of being located in one position, but they could be disordered over several positions. RIGU command in SHELX was used for the refinement of the structures of $\mathbf{1}$ and $\mathbf{2}$. For compound $\mathbf{4}$ the $\mathrm{N}$ atoms were refined as being located in one position, but it is possible that pyridyl rings could be disordered over two positions

and the $\mathrm{C}$ and $\mathrm{N}$ atoms could be sharing the same positions. It was not possible to find a significant difference between $\mathrm{C}$ and $\mathrm{N}$ atoms in this structure based on the X-ray diffraction data. All calculations were performed by the Bruker SHELXL-2014 package..$^{[6]}$ CCDC 1961179 (1) and 1961177 (2) and 1961176 (4) contain the supplementary crystallographic data for this paper. These data can be obtained free of charge from The Cambridge Crystallographic Data Centre via www.ccdc.cam.ac.uk/data_request/cif. 
Crystallographic Data for 1: $\mathrm{C}_{101.83} \mathrm{H}_{64.83} \mathrm{Cl}_{14.48}, \mathrm{C}_{97} \mathrm{H}_{60} \cdot 4.83 \times \mathrm{CHCl}_{3}, \mathrm{M}=1801.70,0.15 \times 0.11 \times$ $0.08 \mathrm{~mm}, \mathrm{~T}=173(2) \mathrm{K}$, Triclinic, space group $P-1, a=20.9020$ (8) $\AA, b=20.9356$ (8) $\AA, c=$ 22.3508(8) $\AA, \alpha=71.185(2)^{\circ}, \beta=88.023(2)^{\circ}, \gamma=83.811(2)^{\circ}, V=9204.0(6) \AA^{3}, Z=4, Z^{\prime}=2, D_{c}=$ $1.300 \mathrm{~g} / \mathrm{cm}^{3}, \mu(\mathrm{Cu})=4.327 \mathrm{~mm}^{-1}, F(000)=3688,2 \theta_{\max }=110.00^{\circ}, 98597$ reflections, 23096 independent reflections [ $R_{\text {int }}=0.0697$ ] $R 1=0.1065, w R 2=0.3295$ and $\mathrm{GOF}=1.044$ for 23096 reflections (1932 parameters) with $\mathrm{I}>2 \sigma(\mathrm{I}), R 1=0.1247, w R 2=0.3545$ and $\mathrm{GOF}=1.037$ for all reflections, max/min residual electron density $+1.124 /-0.565 \mathrm{e}^{-3}$.

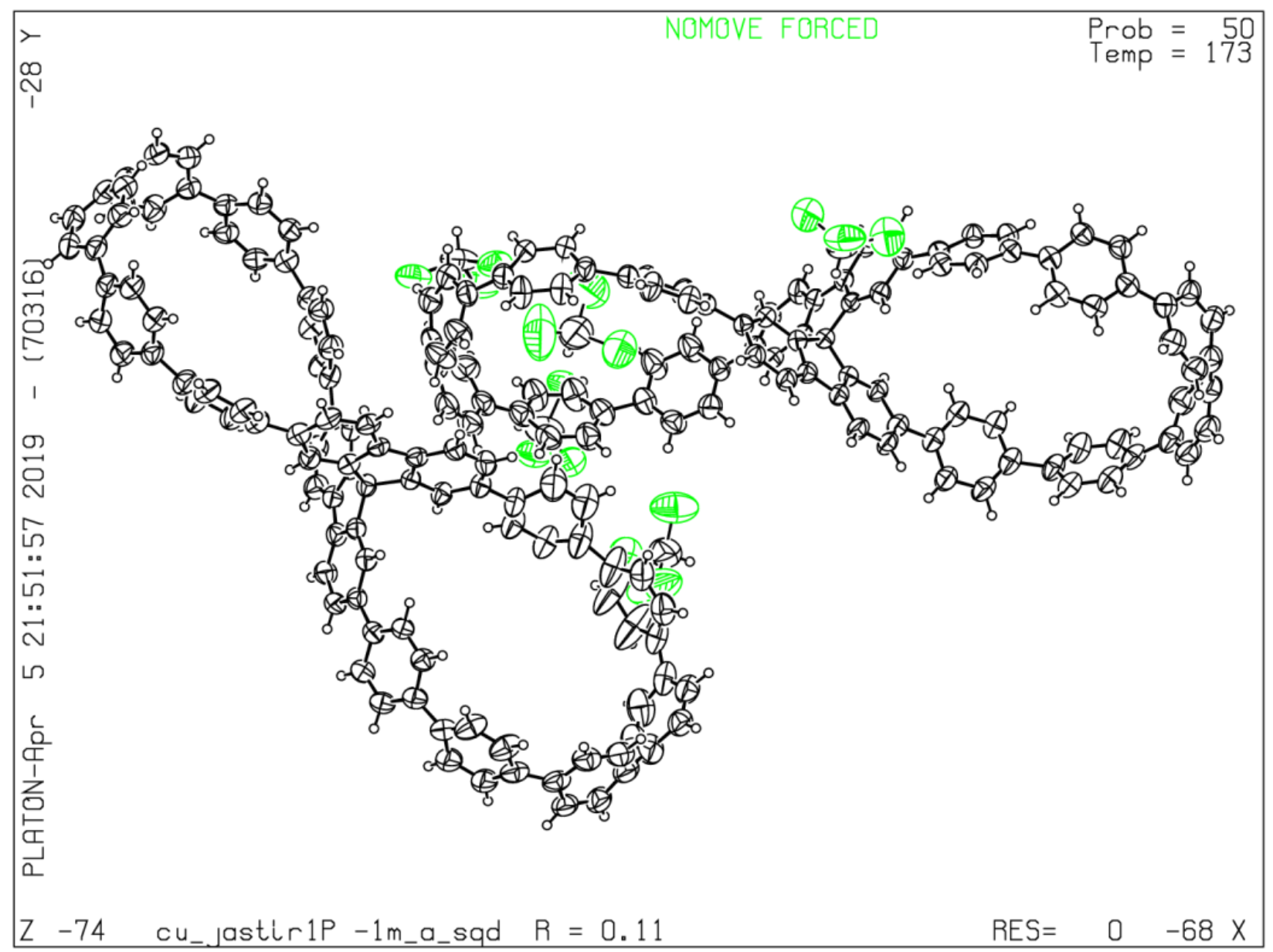

Figure S33: X-ray structure of compound 1 (50\% probability level). 
Crystallographic Data for 2: $\mathrm{C}_{124} \mathrm{H}_{82} \mathrm{Cl}_{6}, \mathrm{C}_{121} \mathrm{H}_{76} \cdot 3 \times \mathrm{CH}_{2} \mathrm{Cl}_{2}, \mathrm{M}=1784.59,0.13 \times 0.06 \times 0.03 \mathrm{~mm}$, $T=173(2) \mathrm{K}$, Monoclinic, space group $P 2_{1} / n, a=18.0821(11) \AA, b=24.2024(15) \AA, c=$ 24.6475(14) $\AA, \beta=110.073(4)^{\circ}, V=10131.3(11) \AA^{3}, Z=4, D_{\mathrm{c}}=1.170 \mathrm{~g} / \mathrm{cm}^{3}, \mu(\mathrm{Cu})=$ $1.920 \mathrm{~mm}^{-1}, F(000)=3712,2 \theta_{\max }=99.70^{\circ}, 37530$ reflections, 10263 independent reflections $\left[R_{\text {int }}\right.$ $=0.0943], R 1=0.1187, w R 2=0.3055$ and $\mathrm{GOF}=1.044$ for 10263 reflections ( 1171 parameters) with $\mathrm{I}>2 \sigma(\mathrm{I}), R 1=0.1996, w R 2=0.3470$ and $\mathrm{GOF}=1.022$ for all reflections, $\mathrm{max} / \mathrm{min}$ residual electron density $+0.465 /-0.496 \mathrm{e}^{-3}$.

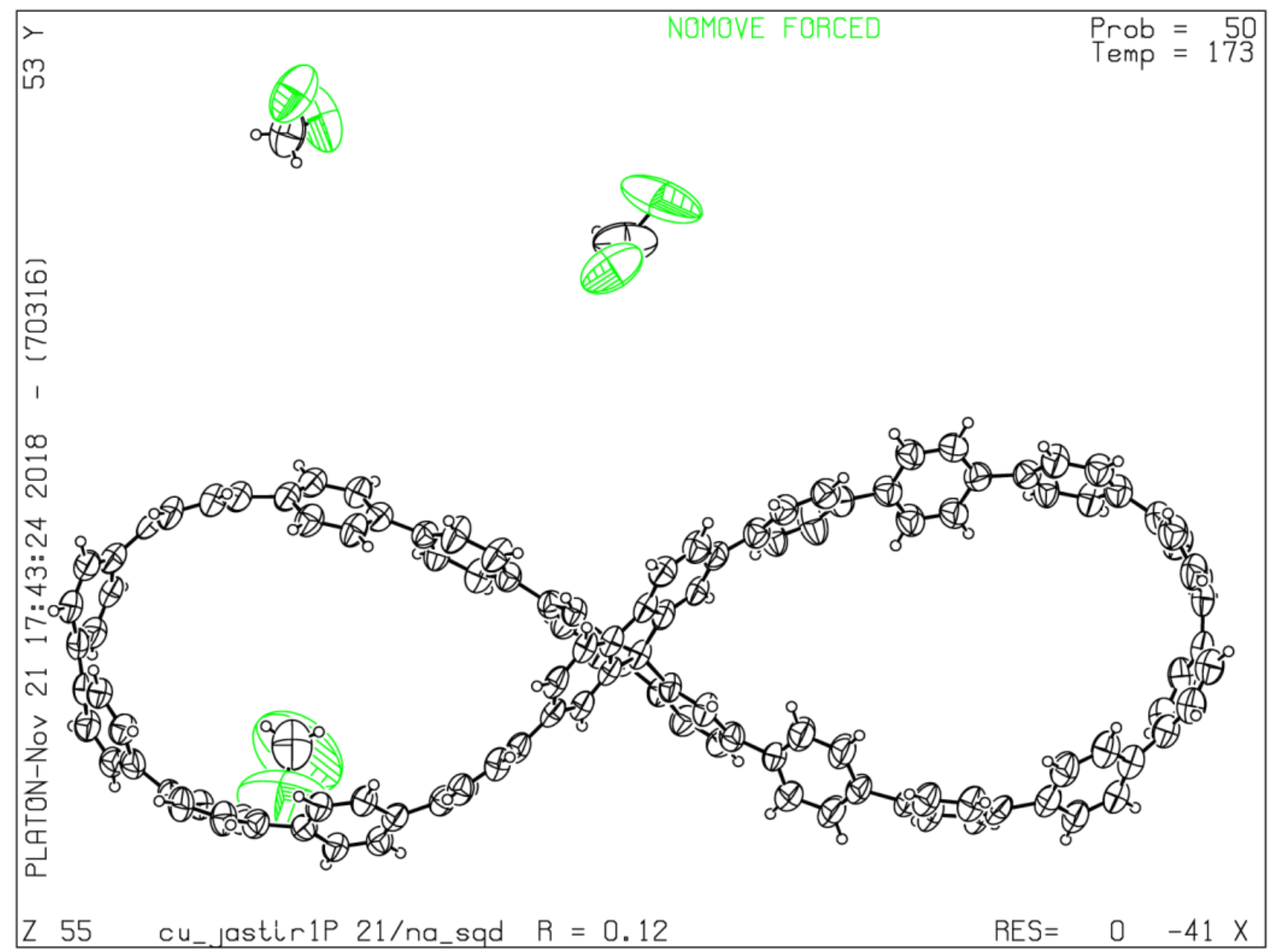

Figure S34: X-ray structure of compound 2 (50\% probability level). 
Crystallographic Data for 4: $\mathrm{C}_{123} \mathrm{H}_{116} \mathrm{~N}_{4} \mathrm{O}_{7.50}, \mathrm{C}_{93} \mathrm{H}_{56} \mathrm{~N}_{4} \cdot 7.5 \times \mathrm{C}_{4} \mathrm{H}_{8} \mathrm{O}, \mathrm{M}=1770.19,0.22 \times 0.08 \times$ $0.02 \mathrm{~mm}, \mathrm{~T}=173(2) \mathrm{K}$, Monoclinic, space group $C 2 / c, a=38.384(3) \AA$, $b=15.8148(13) \AA, c=$ $32.575(3) \AA, \beta=93.970(5)^{\circ}, V=19727(3) \AA 33, Z=8, D_{c}=1.192 \mathrm{~g} / \mathrm{cm}^{3}, \mu(\mathrm{Cu})=0.572 \mathrm{~mm}^{-1}, F(000)$ $=7536,2 \theta_{\max }=133.83^{\circ}, 54002$ reflections, 17075 independent reflections $\left[R_{\text {int }}=0.0527\right], R 1=$ $0.0876, w R 2=0.2435$ and GOF $=1.005$ for 17075 reflections ( 1099 parameters) with $\mathrm{I}>2 \sigma(\mathrm{I}), R 1$ $=0.1416, w R 2=0.2743$ and GOF $=1.006$ for all reflections, $\max / \mathrm{min}$ residual electron density $+0.347 /-0.328 \mathrm{e}^{-3}$.

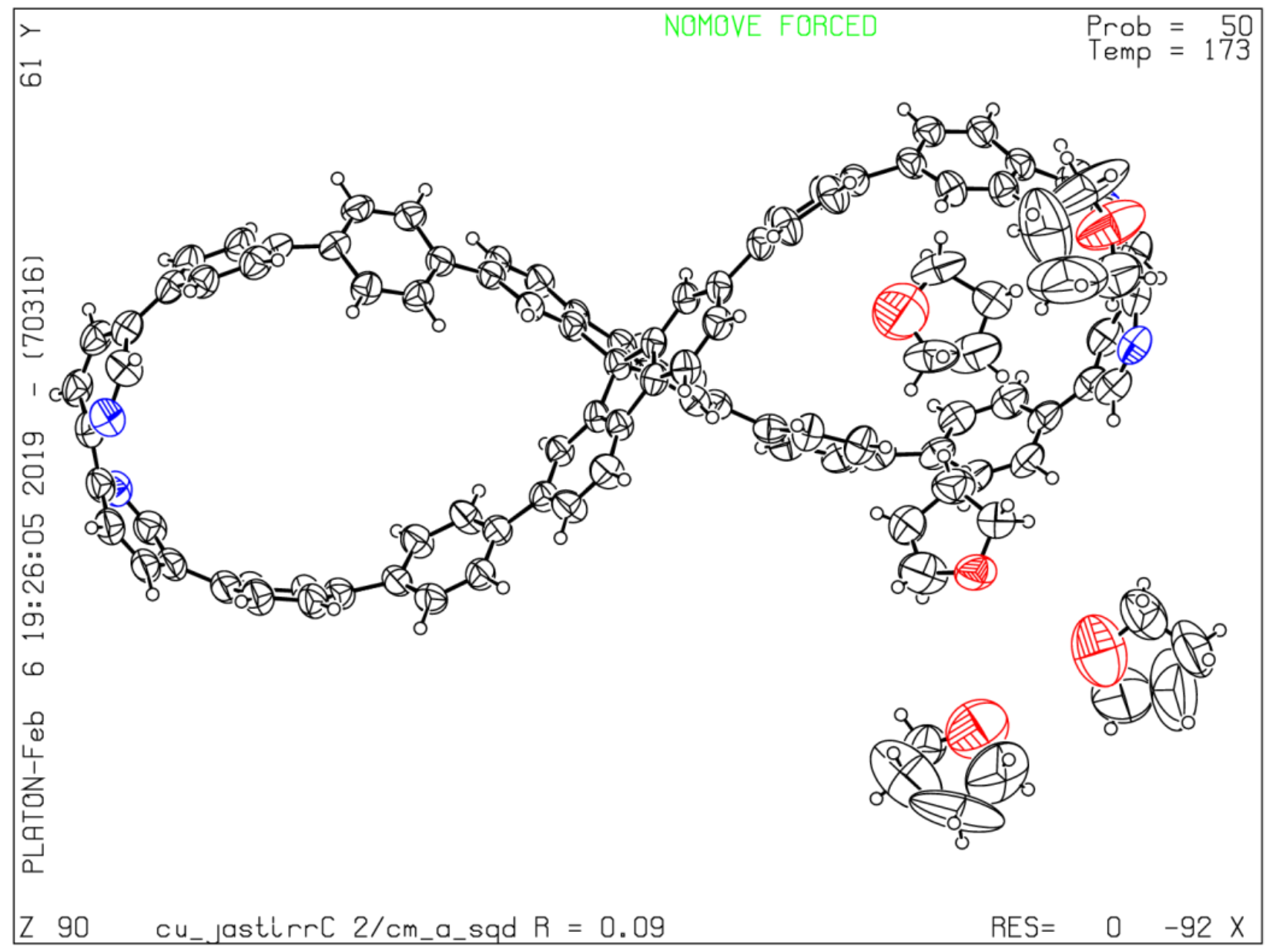

Figure S35: X-ray structure of compound 4 (50\% probability level). 

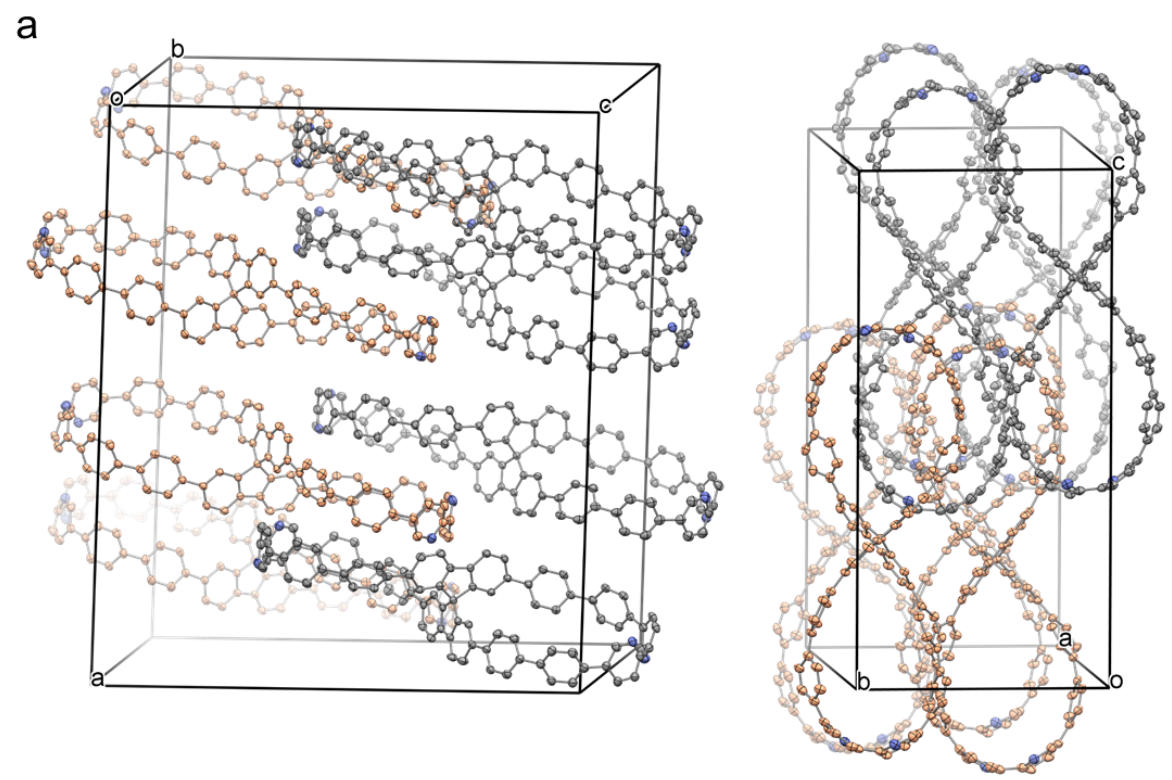

b

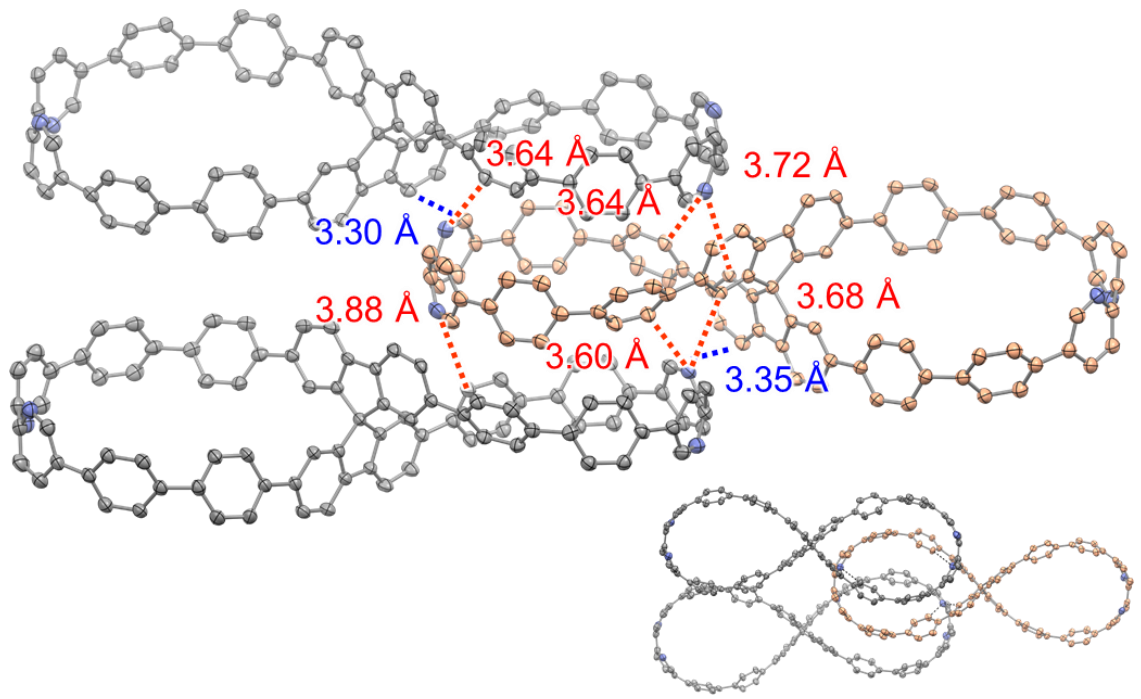

Figure S36: Unit cell of 4 (50\% probability level, solvent molecules omitted, selected lemniscates are colored orange for clarity, a) and solid state intermolecular interactions of the pyridyl nitrogen (red) and $\pi-\pi$ stacking (blue) denoted by short contacts (b). 


\section{Gas Sorption Experiments}

$\mathrm{N}_{2}$ uptake measurements were performed using a Micromeritics ASAP 2020 Plus. Samples were prepared by dissolving the respective compound in minimal amounts of $\mathrm{CH}_{2} \mathrm{Cl}_{2}$ and rapid precipitation by addition of pentane. The precipitate was filtered off and dried at room temperature under vacuum for $16 \mathrm{~h}$. Prior to analysis, powder samples were further evacuated to $2 \mu$ torr and held at $200^{\circ} \mathrm{C}$ for approximately 48 hours. The temperatures were determined by the sample's thermal stability as determined by thermal gravimetric analysis. Activation was considered complete when the outgassing rate fell below $2.5 \mu$ torr $\mathrm{min}^{-1}$. The sample mass was determined by the difference in mass between the empty sample tube and the loaded sample tube postevacuation. $\mathrm{N}_{2}$ uptake was measured isothermally at $77 \mathrm{~K}$. Pressure ranges for BET surface area analysis were selected based on guidelines detailed previously. ${ }^{[7]}$

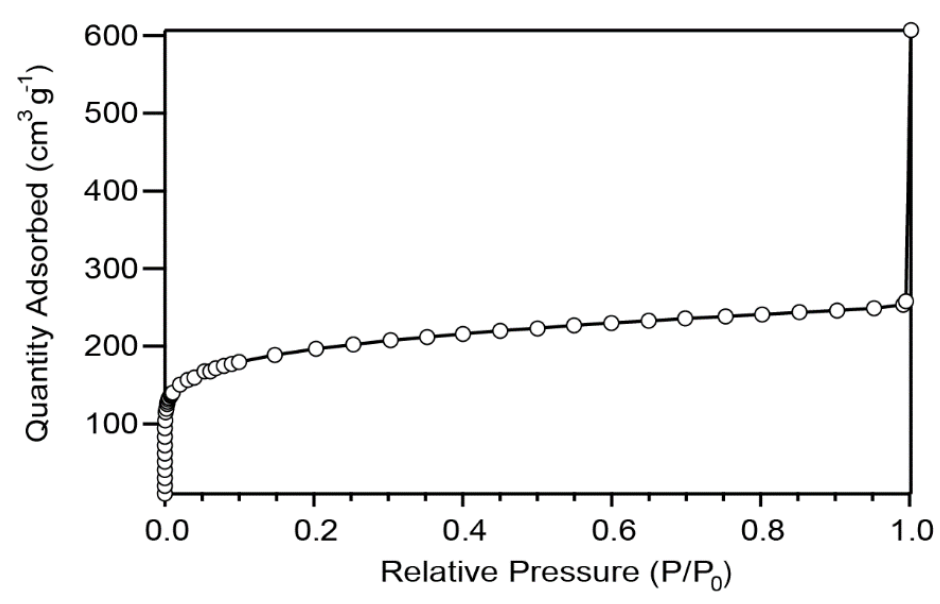

Figure S37: $\mathrm{N}_{2}$ uptake isotherm of 2 collected at $77 \mathrm{~K}$. Prior to analysis, the sample was evacuated to 2 micro torr at $200{ }^{\circ} \mathrm{C}$ for approximately 48 hours.

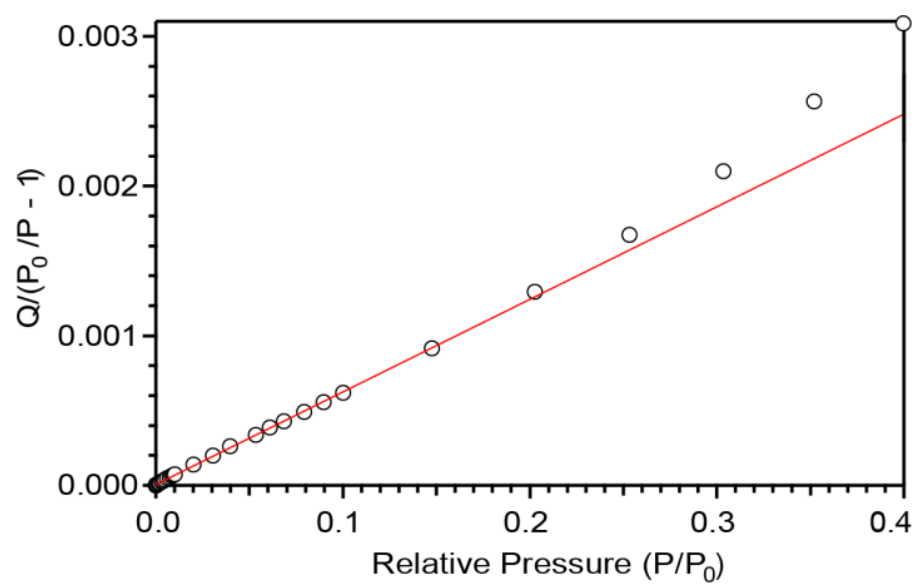

Figure S38: Brunauer-Emmett-Teller (BET) plot of 2. Analysis was based on a linear fit (shown in red) to $\mathrm{N}_{2}$ isotherm data at relative pressures between $10^{-7}$ and $10^{-1} \mathrm{P} / \mathrm{P}_{0}$. 
Table S1: Tabular plot of $\mathrm{N}_{2}$ uptake data for 2 . BET analysis was performed using the data shown in italics.

Relative Pressure

Quantity Adsorbed (cm $3 / \mathrm{g}$ $\left(P / P_{0}\right)$ STP)

\begin{tabular}{|c|c|}
\hline $7.68 E-07$ & 10.11063 \\
\hline 4.76E-06 & 20.38063 \\
\hline $1.01 E-05$ & 30.68431 \\
\hline $1.46 E-05$ & 41.10018 \\
\hline $2.22 E-05$ & 51.53947 \\
\hline 3.87E-05 & 62.01334 \\
\hline $6.41 E-05$ & 72.73473 \\
\hline 0.000134 & 83.46312 \\
\hline 0.000254 & 94.26093 \\
\hline 0.000577 & 104.8549 \\
\hline 0.001303 & 115.0029 \\
\hline 0.001937 & 119.3461 \\
\hline 0.003055 & 125.2867 \\
\hline 0.003925 & 128.5494 \\
\hline 0.00483 & 131.1728 \\
\hline 0.005985 & 133.9816 \\
\hline 0.007422 & 135.7521 \\
\hline 0.008389 & 137.5987 \\
\hline 0.009307 & 139.0443 \\
\hline 0.010104 & 140.1073 \\
\hline 0.020276 & 150.5386 \\
\hline 0.030335 & 156.8722 \\
\hline 0.039771 & 159.6207 \\
\hline 0.053414 & 167.9496 \\
\hline 0.060989 & 167.7226 \\
\hline 0.06828 & 171.2895 \\
\hline 0.079176 & 174.7448 \\
\hline 0.089725 & 177.4162 \\
\hline 0.100096 & 180.0336 \\
\hline 0.147565 & 189.0064 \\
\hline 0.202734 & 196.7734 \\
\hline 0.253329 & 202.472 \\
\hline 0.303439 & 207.5013 \\
\hline 0.352116 & 211.9007 \\
\hline 0.39984 & 215.8564 \\
\hline 0.44983 & 219.8567 \\
\hline 0.499954 & 223.4337 \\
\hline 0.54943 & 226.782 \\
\hline 0.599567 & 229.9796 \\
\hline 0.649435 & 232.8646 \\
\hline 0.699041 & 235.9536 \\
\hline 0.752622 & 238.579 \\
\hline 0.80176 & 241.0737 \\
\hline 0.852291 & 243.6898 \\
\hline 0.9024 & 246.1791 \\
\hline 0.952317 & 248.9 \\
\hline 0.991027 & 253.4229 \\
\hline 0.994875 & 257.6594 \\
\hline 1.00151 & 606.6075 \\
\hline
\end{tabular}




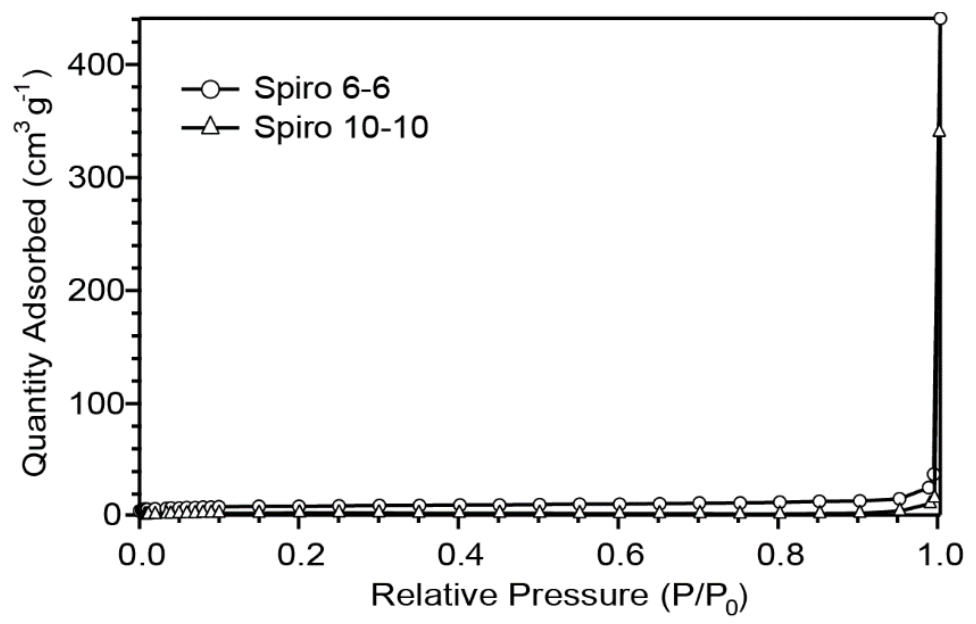

Figure S39: $\mathrm{N}_{2}$ uptake isotherms of 1 and 3 collected at $77 \mathrm{~K}$. Prior to analysis, the sample was evacuated to 2 micro torr at $200{ }^{\circ} \mathrm{C}$ for approximately 48 hours.

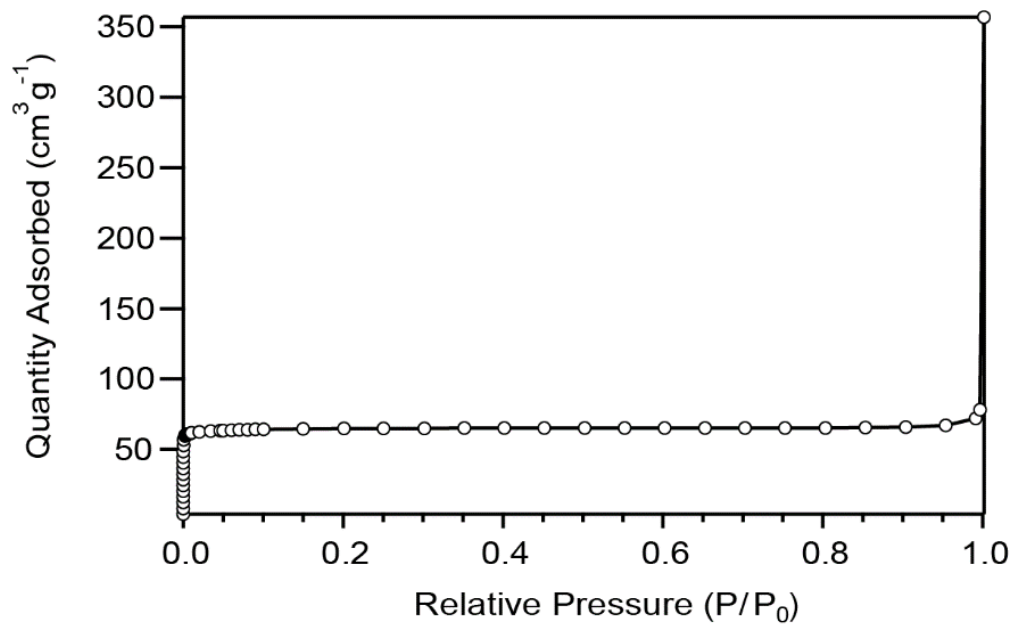

Figure S40: $\mathrm{N}_{2}$ uptake isotherm of [9[CPP. Prior to analysis, the sample was evacuated to $2 \mu$ torr at 200 ${ }^{\circ} \mathrm{C}$ for approximately 48 hours.

Table S2: Tabular plot of $\mathrm{N}_{2}$ uptake data for [9[CPP.

\begin{tabular}{|c|c|c|}
\hline $\begin{array}{l}\text { Relative } \\
\left(\mathbf{P} / \mathbf{P}_{0}\right)\end{array}$ & $\begin{array}{l}\text { Quantity } \\
\text { STP) }\end{array}$ & Adsorbed \\
\hline $8.94288 e-08$ & & 4.09181 \\
\hline $1.85494 \mathrm{e}-08$ & & 8.18693 \\
\hline $1.02762 \mathrm{e}-08$ & & 12.2781 \\
\hline $1.10584 \mathrm{e}-08$ & & 16.3669 \\
\hline $4.68839 e-05$ & & 20.3877 \\
\hline 0.000124495 & & 24.3504 \\
\hline 0.000150719 & & 28.3939 \\
\hline 0.000161555 & & 32.4616 \\
\hline 0.000175441 & & 36.5245 \\
\hline
\end{tabular}




\begin{tabular}{lc}
0.000186248 & 40.5939 \\
0.000201267 & 44.6558 \\
0.000225917 & 48.7027 \\
0.000283982 & 52.8283 \\
0.000500959 & 56.9 \\
0.00191395 & 59.7266 \\
0.0022103 & 59.9081 \\
0.00321225 & 60.347 \\
0.00399291 & 60.5803 \\
0.00499655 & 60.8035 \\
0.00601047 & 60.9946 \\
0.00711797 & 61.1588 \\
0.00805578 & 61.33 \\
0.00922765 & 61.5856 \\
0.0101255 & 61.7656 \\
0.0199196 & 62.4087 \\
0.0342343 & 62.9337 \\
0.0459664 & 63.2991 \\
0.0500258 & 63.4875 \\
0.0605315 & 63.7486 \\
0.0701658 & 63.9422 \\
0.0802196 & 64.1418 \\
0.0902057 & 64.3069 \\
0.100526 & 64.4238 \\
0.149926 & 64.6531 \\
0.20094 & 64.8428 \\
0.250659 & 65.0083 \\
0.301556 & 65.0815 \\
0.351296 & 65.1441 \\
0.401623 & 65.1963 \\
0.451812 & 65.1992 \\
0.501968 & 65.2233 \\
0.552212 & 65.2323 \\
0.602289 & 65.2237 \\
0.652518 & 65.2068 \\
0.702754 & 65.2171 \\
0.75276 & 65.2276 \\
0.802987 & 65.3283 \\
0.853314 & 65.5414 \\
0.903453 & 65.9409 \\
\hline & \\
\hline
\end{tabular}




\section{Powder X-ray Diffraction}
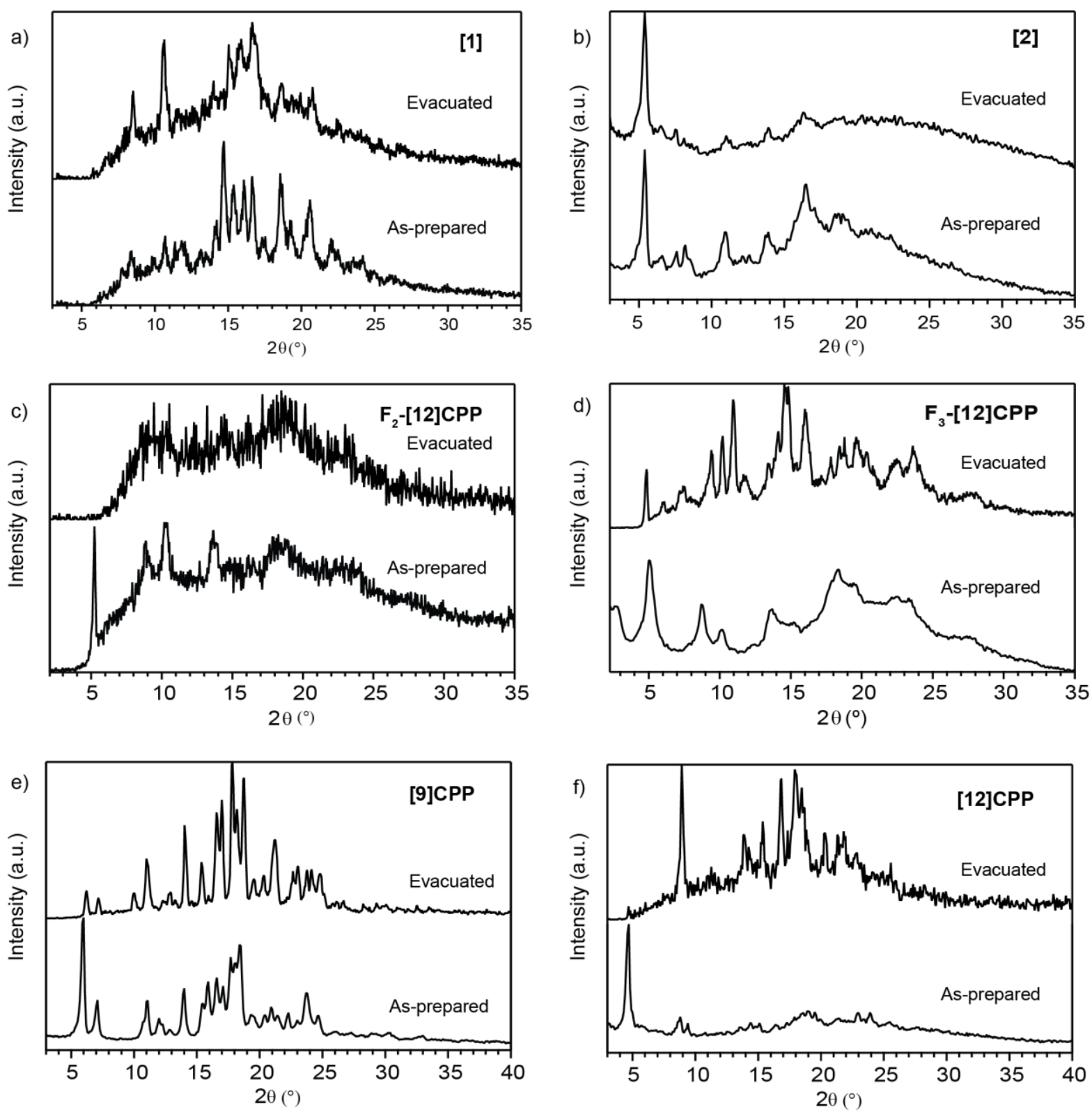

Figure S41: PXRD patterns of products before and after evacuation procedure. Samples was evacuated at $200{ }^{\circ} \mathrm{C}$ for 48 hours, or until the degas rate was below $2.5 \mu$ torr min $^{-1}$. The samples $2, F_{3}-[12] \mathrm{CPP}$, and [9]CPP maintain crystalline order (b, d, e). The samples 1 and [12]CPP change structure significantly, although the evacuated products still maintain some crystallinity $(a, f)$. The sample $F_{2}-[12] C P P$ loses crystallinity during the evacuation process (c). The sample 3 is completely amorphous before and after the evacuation. 


\section{Quartz Crystal Microbalance Measurements}

\section{General Information}

QCM measurements were carried out by a reported protocol: ${ }^{[8]}$ Nitrogen used in the screening experiments has a purity of $99.998 \%$. Reagents for the pretreatment of the quartz crystal microbalances: $1 H, 1 H, 2 H, 2 H$-perfluorooctyl-1-phosphonic acid (98\%, ABCR, FP-8). The precoating of the passivating layer with FP-8 was performed with a concentration of $1.5 \mathrm{mmol} / \mathrm{L}$ in ethanol for $24 \mathrm{~h}$ and washing the QCMs several times with pure ethanol. ${ }^{[9]}$ The experimental primary data were processed by Matlab R2017b (The MathWorks Inc., Natrick, Massachusetts, USA). For the preparation of the diagrams Origin 8 SR0 (OriginLab Corporation, Northampton, Massachusetts, USA) was used. HFF-QCMs with a fundamental frequency of $195 \mathrm{MHz}$ were employed (KVG Quartz Crystal Technology $\mathrm{GmbH}$, Neckarbischofsheim, Germany. Type: XA 1600). ${ }^{[10]}$ The QCM is excited using an aperiodic oscillator circuit and oscillates with its specific load resonance frequency. Frequency counting is performed using a FPGA (field programmable gate array) which allows asynchronous 28 -bitcounting with an accuracy of $\pm 0.5 \mathrm{~Hz}$. ${ }^{11]}$

\section{Quartz Crystal Microbalances (QCM) Setup}

The resonance frequency of thickness-shear resonators, such as the quartz crystal microbalances employed, is largely influenced by the oscillating mass. The sensitivity of the quartz resonator is influenced by several environmental factors and is described by the Sauerbrey equation (equation E1). ${ }^{[12]}$ Wherein $A$ is the oscillating area, $N$ the frequency constant, and $\rho$ the density of the quartz material. A variation in the oscillating mass $\Delta m$ directly results in a linear shift $\Delta f_{0}$ of the fundamental resonance frequency $t_{0}$.

$$
\Delta f_{0}=\frac{-f_{0}^{2}}{N \cdot \rho \cdot A} \cdot \Delta m
$$

This correlation allows determining adsorption from various analytes towards selected affinity materials. ${ }^{[13]}$ The QCM is excited using an aperiodic oscillator circuit and oscillates with its specific load resonance frequency. ${ }^{[14]}$ Frequency counting is performed using a FPGA (field programmable gate array) which allows asynchronous 28-bit counting with an accuracy of $\pm 0.5 \mathrm{~Hz}$. For testing purposes, a larger number of electronic circuits and QCMs can be combined to operate in a single setup.

\section{Coating Protocol}

The coating of the quartz crystal microbalances is performed using an electrospray protocol. ${ }^{[15]}$ This particular method is well established and allows the continuously monitoring of mass deposition onto the quartz upon spraying process. The electrospray solutions are prepared at concentrations of approx. $0.1 \mathrm{mg} \cdot \mathrm{mL}^{-1}$ in a $9: 1$ mixture of tetrahydrofuran/methanol. The experimental setup for the coating unit is schematically displayed in Figure S42 and Figure S43.The solution for coating is placed in a glass syringe equipped with a metal cannula. The metallic needle is contacted with an applied voltage of $5 \mathrm{kV}$ relative to a counter electrode which 
is represented by the electrode of the QCM to be coated (distance needle tip/electrode: approx. $0.25 \mathrm{~m}$ ). The cannula represents the anode. A constant delivery of the solution during the coating process is achieved by using a syringe pump $\left(3 \mu \mathrm{L} \cdot \mathrm{min}^{-1}\right)$. The coating process is monitored by measuring the frequency shift of the QCM. Since it is not possible to measure the thickness of the deposited film directly, the amount of affinity material on the quartz device is given as a frequency shift. For screening purposes, all compounds are deposited on $195 \mathrm{MHz}$ QCMs until a frequency shift of $50 \mathrm{kHz}$ is reached. This shift corresponds to a mass of $10.4 \mathrm{ng}$ of the deposited material on the oscillating QCM section.

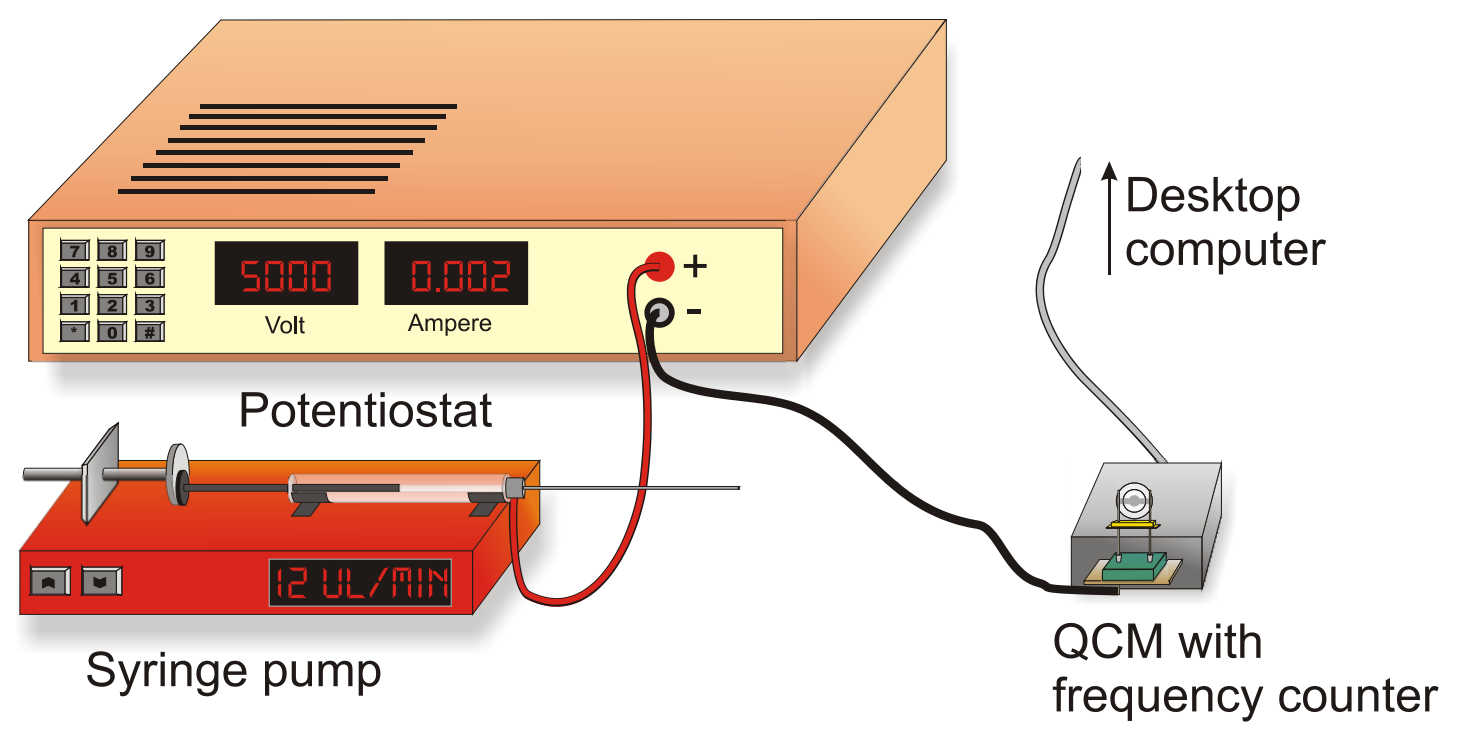

Figure S42: Schematic of the setup for ESI coating with affinity materials. ${ }^{[8]}$ 


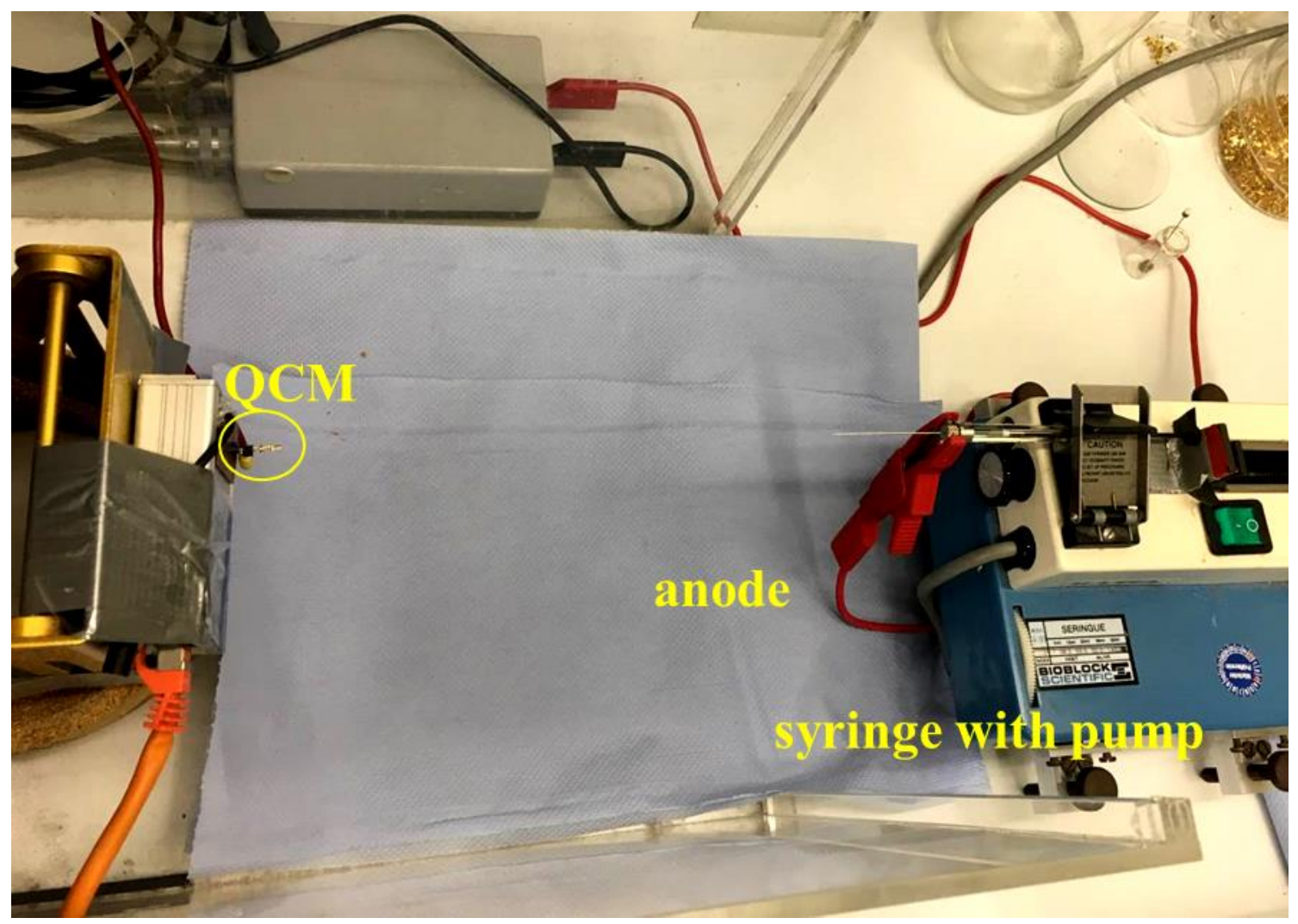

Figure S43: ESI setup used in the lab. ${ }^{[16]}$

\section{Setup for Measurement}

For the determination of affinities, conditions and concentration of analytes need to be controlled precisely. Therefore, a "closed" system connected to a gas mixing unit is used (Figure S44). In this gas mixing unit, the inert nitrogen flow is divided into two streams, both controlled by individual mass flow controller (MFC) from the Brooks Instrument company (Model 5050S). Gasflow 1 remains unchanged in temperature and composition and is used as gas source for dilution purposes. Whereas, flow 2 is bubbled through an interchangeable analyte-reservoir which is adjusted to $293.1 \pm 0.2 \mathrm{~K}$. The analyte-saturated gas flow 2 is mixed with the pure inert gas flow 1 and reaches to the measuring chamber. The overall gas flow is set to $200 \mathrm{~mL} \mathrm{~min}^{-1}$. By carefully controlling the flow of both streams by the MFCs, it is possible to produce gas mixtures with concentrations of $1 \%$ to $100 \%$ of the vapor pressure of the pure analyte at $293.1 \pm 0.2 \mathrm{~K}$. Piperonal and $(-)$-norephedrine were measured at $298.1 \pm 0.2 \mathrm{~K}$. The central part of the screening set-up is the measuring cell which is connected to the gas mixing unit and placed in a temperature adjusted environment. We employed a slightly modified GC oven (Hewlett Packard, Palo Alto, CA, USA. Type: HP 5890). The cell is kept constantly at $308 \mathrm{~K}$ to exclude temperature influences and to prevent condensation effects within the cell. The cell is designed to operate up to 12 QCMs in a parallel fashion. 


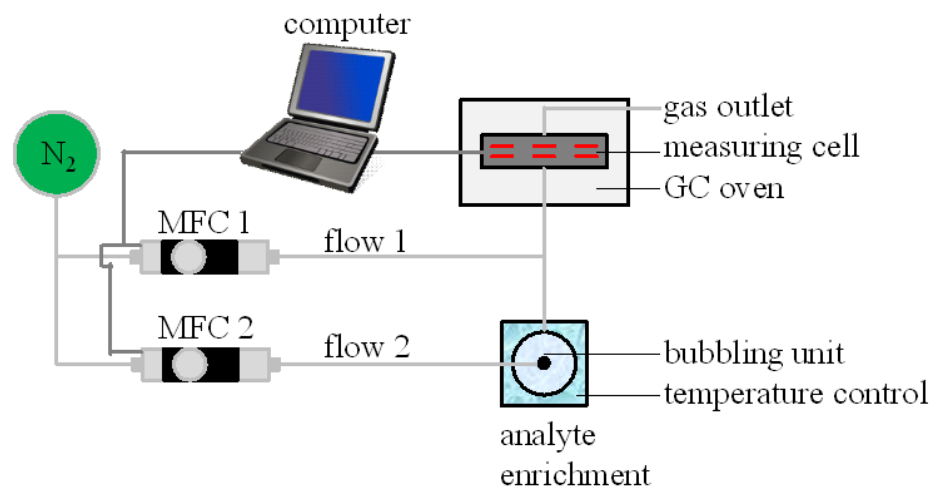

Figure S44: Schematic of the setup used for measurements. ${ }^{[8]}$

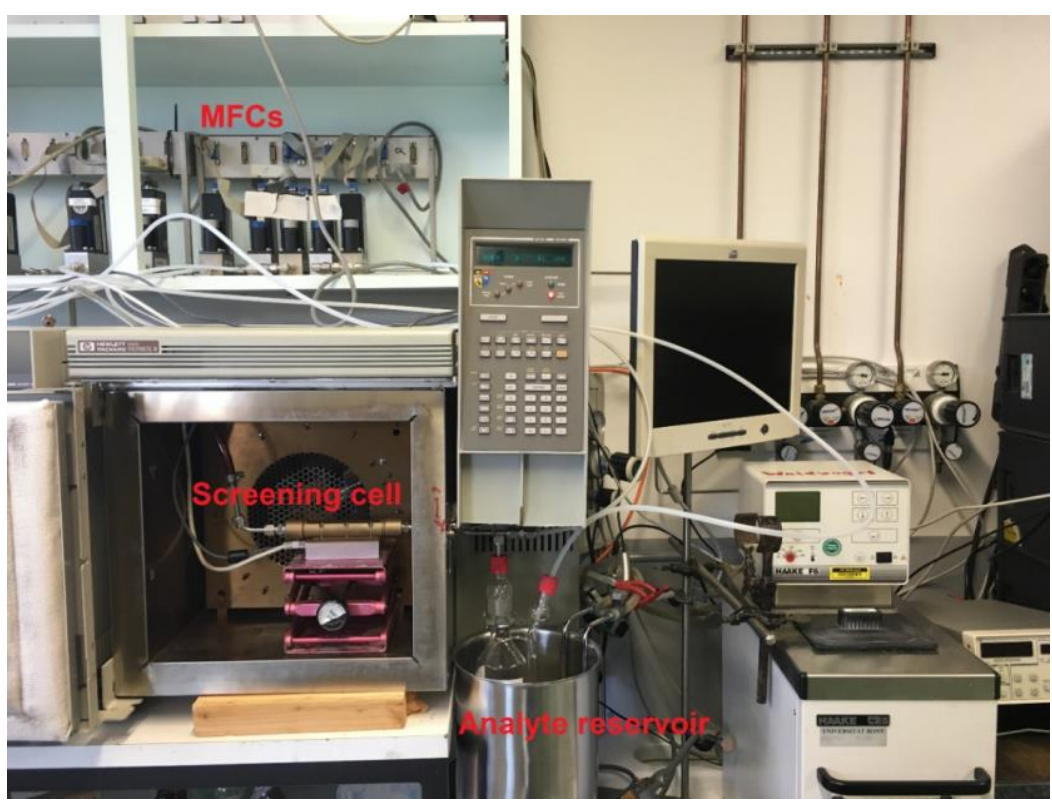

Figure S45: Setup for measurement used in the lab.[8] 


\section{Screening Experiments}

In the following the procedure used in our lab to determine the affinity is exemplified. This procedure is carried out for every affinity material with the individual analyte. In Figure S46, a typical measurement of an analyte is shown. The frequency shifts correspond to the different analyte concentration. In a complete measurement the steps of concentration are $1 \%, 2 \%, 5 \%$, $10 \%, 15 \%, 20 \%, 25 \%, 35 \%$, and $50 \%$ of the saturated analyte vapor.

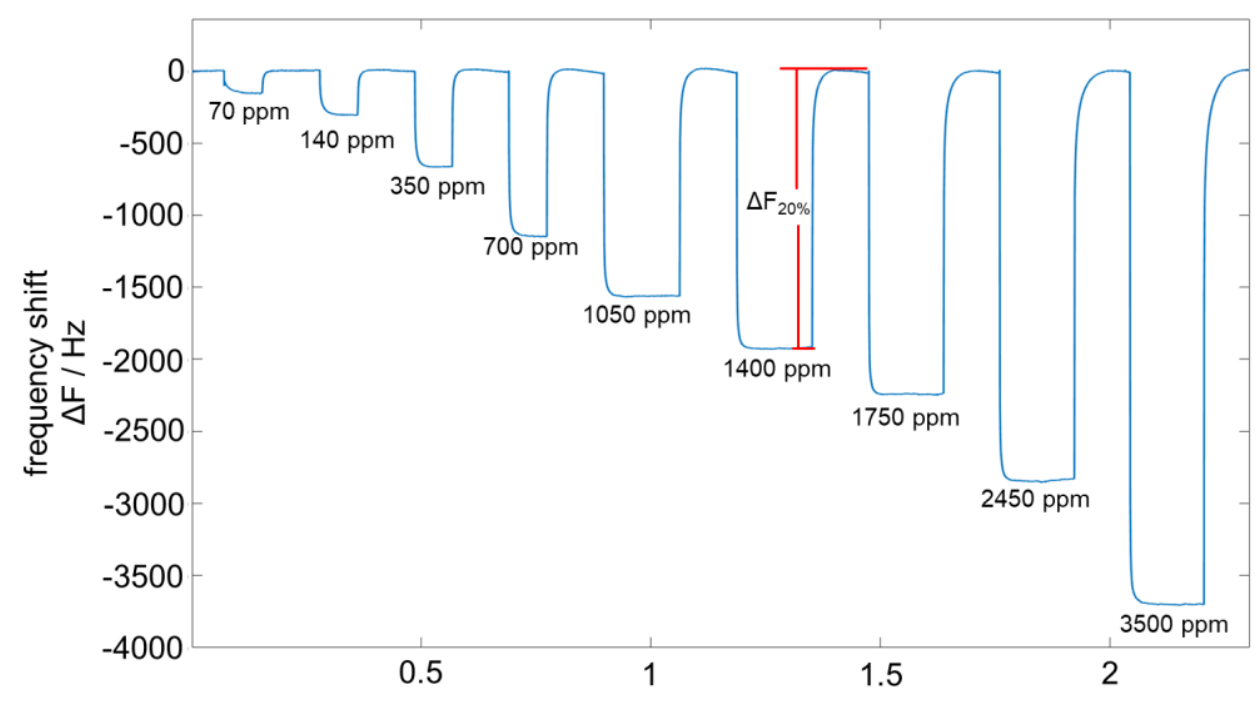

time $/ 10^{-4} \mathrm{~s}$

Figure S46: Typical frequency shifts for measurements of the affinity material $\mathbf{3}$ with o-xylene at different concentrations in the nitrogen flow.

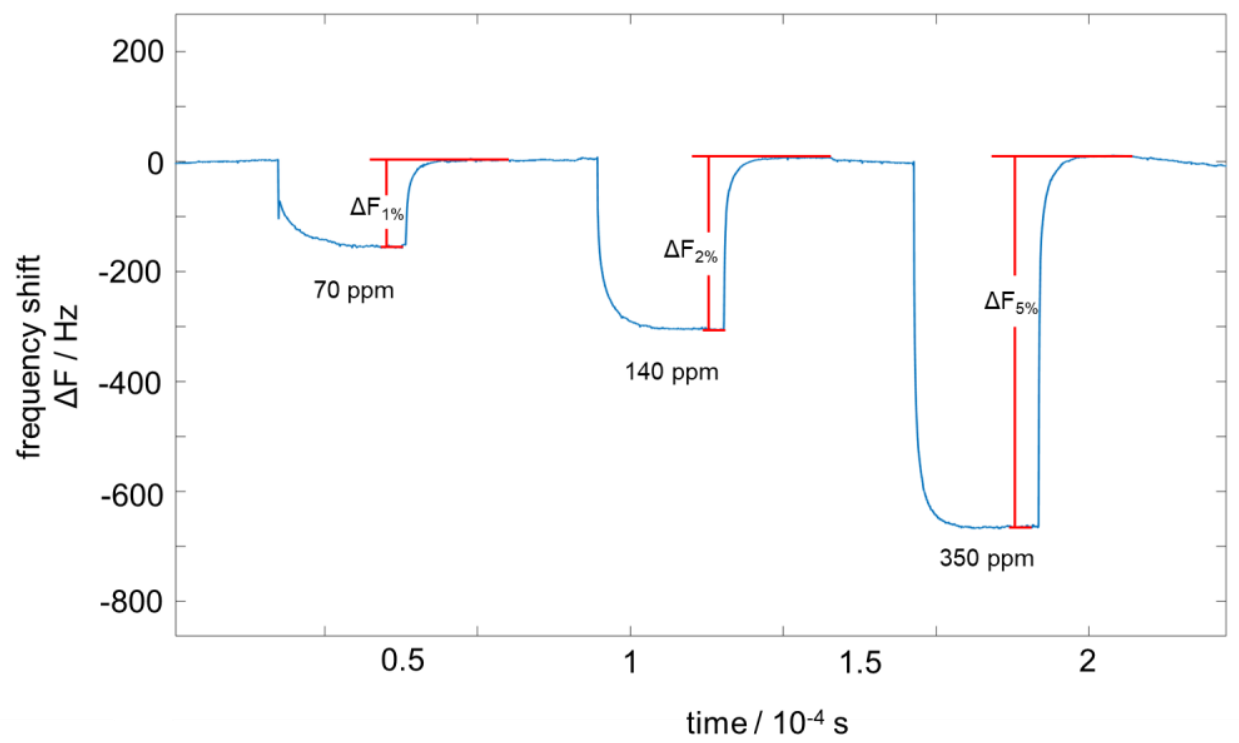

Figure S47: Detailed evaluation of frequency shifts for affinity material 3 and 0 -xylene at concentrations $70 \mathrm{ppm}(1 \%)$, 140 ppm (2\%) and 350 ppm (5\%). 


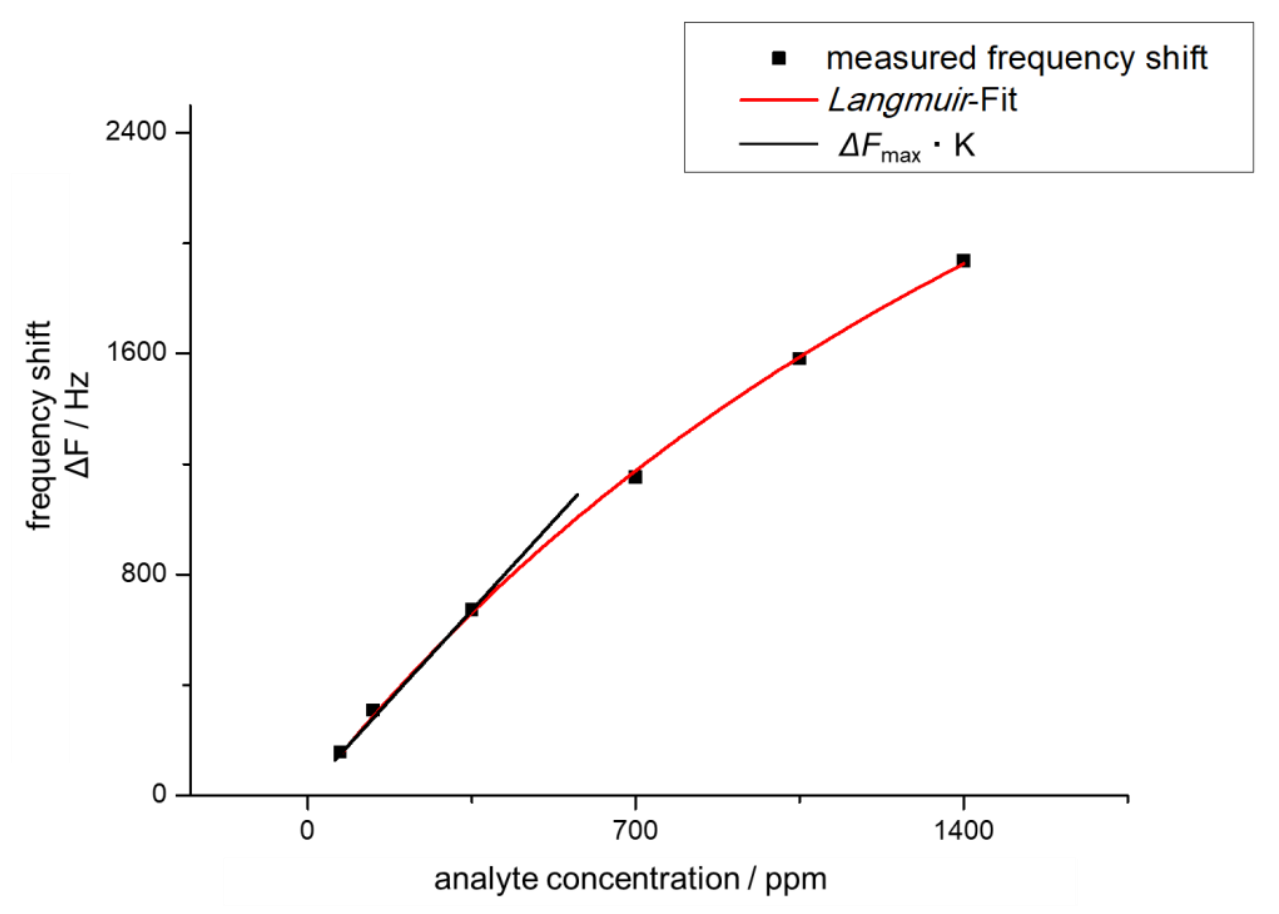

Figure S48: Affinity determination from the frequency shifts for different concentrations.

By plotting the frequency shift (measured as shown in Figure S47) vs. the analyte concentration, the constants of the Langmuir adsorption isotherm were determined (Figure S48). The graph is obtained by fitting to equation E2.

$$
\Delta F=\frac{\Delta F_{\text {max }} \cdot K \cdot c_{\text {analyte }}}{1+K \cdot c_{\text {analyte }}}
$$

The slope of the linear part of the Langmuir equation is the product of the Langmuir constants in equation E2. Since the resulting value is a general information about the affinity of a film or porous material to a respective analyte, $\Delta F \max \cdot K$ is used as the affinity within this study.

Table S3: Overview of measured analytes.

\begin{tabular}{lcc} 
Analyte & $\begin{array}{c}\text { Vapor pressure }\left(\text { at } \mathbf{2 0}{ }^{\circ} \mathbf{C}\right) \\
{[\mathbf{p p m}]}\end{array}$ & $\begin{array}{c}\text { Applied concentration range } \\
\text { [ppm] }\end{array}$ \\
\hline TNT & $0.27^{[\mathrm{J}]}$ & $0.00005-0.0025$ \\
$2,4-D N T$ & $0.29^{[\mathrm{G}]}$ & $0.0020-0.10$ \\
1,3,5-triethylbenzene & $0.35^{[\mathrm{C}] *}$ & $0.0035-0.175$ \\
1,2-diethylbenzene & $0.92^{[\mathrm{C}] *}$ & $0.0092-0.451$ \\
1,3-diethylbenzene & $1.02^{[\mathrm{C}] *}$ & $0.0102-0.501$ \\
1,4-diethylbenzene & $1.03^{[\mathrm{C}] *}$ & $0.0103-0.502$ \\
TATP & $18.5^{[\mathrm{F}]}$ & $0.185-9.25$ \\
acetophenone & $211^{[\mathrm{D}]}$ & $2.1-105.5$
\end{tabular}


GBL

pseudocumene

nitrobenzene

$o$-xylene

$m$-xylene

$p$-xylene

$\mathrm{H}_{2} \mathrm{O}$

toluene

benzene

cyclohexane

acetone
$1500^{[E]}$

$2300^{[\mathrm{A}]}$

$300^{[\mathrm{H}]}$

$7000^{[\mathrm{B}]}$

$8000^{[\mathrm{A}]}$

$8700^{[\mathrm{B}]}$

$14500^{[B]}$

$29331^{[\mathrm{A}]}$

$99458^{[\mathrm{A}]}$

$103000^{[\mathrm{B}]}$

$245310^{[B]}$
15-750

23-1150

3-150

70-3500

$80-4000$

87-4350

$145-7250$

293.31-14665

994.58-49729

1030-51500

2453.1-122655

*calculated values; References for vapor pressures: [A] Handbuch Feinchemikalien, Aldrich, 2010. [B] Chemikalien \& Reagenzien, Merck KGaA, Darmstadt, 2011. [C] D. R. Lide, CRC Handbook of chemistry and physics, 87. ed., Taylor \& Francis Group, Boca Raton, 2006. [D] Cayman Chemical, SDS Acetophenone, URL: https://www.caymanchem.com/msdss/16444m.pdf (downloaded: 18.04.2016). [E] International Chemical Safety Card (ICSC) of $\mathrm{y}$-Butyrolactone, URL: http://www.ilo.org/dyn/icsc/showcard.display?p_version=2\&p_card_id=1020 (downloaded: 04.02.2020). [F] C. Oxley, J. Smith, K. Shinde, J. Moran, Propellants, Explosives, Pyrotechnics 2005, 30, 127-130. [G] D. H. Rosenblatt, E. P. Burrows, W. R. Mitchell, D. L. Parmer in The Handbook of Environmental Chemistry (Eds.: O. Hutzinger, F. Brochhagen, E. P. Burrows, H. Fiedler, J. Konietzko, W. R. Mitchell, K. Mross, W. Mücke, D. L. Parmer, D. H. Rosenblatt), Springer Berlin Heidelberg, Berlin, Heidelberg, 1991. [H] nitrobenzene: GHS Material Safety Data Sheet, Merck, URL: https://www.sigmaaldrich.com/MSDS/MSDS/DisplayMSDSPage.do?country=DE\&language=ENgeneric\&productNumber=06084\&brand=SIAL\&PageToGoToURL=https\%3A\%2F\%2Fwww. sigmaaldrich.com\%2Fcatal og\%2Fproduct\%2Fsial\%2F06084\%3Flang\%3Dde (downloaded: 19.06.2019). [I] Chemspider Database: calculated with ACD/Labs: http://www.chemspider.com/Chemical-Structure.21171503.html (downloaded 19.06.2019). [J] Y. Choi, S. Jeong, H. Ryu, K. Lee, B. H. Bae, K. Nam, Hum. Ecol. Risk Assess. 2011, 17, 856-872. 


\section{Screening Results}

In order to obtain comparable results, the coating of the QCM was always made with the same mass of affinity material ( $50 \mathrm{kHz}$ which corresponds to approx. $10.4 \mathrm{ng}$ ). By that, a comparable film thickness is achieved. In the following tables, the affinity is presented for the analytes mentioned in the former chapter. All affinities were obtained by the procedure described before.

Table S4: Affinities of FP-8, CPPs, $p$-quinquephenyl and affinity materials 1-4 towards benzene, toluene, $o$-xylene, $m$ xylene and $p$-xylene.

\begin{tabular}{|c|c|c|c|c|c|}
\hline $\begin{array}{l}\text { Affinity } \\
{\left[\mathrm{Hz} \cdot \mathrm{ppm}^{-1}\right]}\end{array}$ & benzene & toluene & o-xylene & $m$-xylene & $p$-xylene \\
\hline FP-8 & 0.058 & 0.169 & 0.565 & 0.543 & 0.540 \\
\hline$\Delta \mathrm{FP}-8$ & 0.001 & 0.002 & 0.006 & 0.007 & 0.002 \\
\hline p-quinquephenyl & 0.069 & 0.209 & 0.661 & 0.617 & 0.561 \\
\hline$\Delta p$-quinquephenyl & 0.001 & 0.005 & 0.017 & 0.020 & 0.003 \\
\hline [6]CPP & 0.102 & 0.375 & 1.041 & 0.988 & 1.099 \\
\hline$\Delta[6] \mathrm{CPP}$ & 0.002 & 0.004 & 0.038 & 0.041 & 0.005 \\
\hline [9]CPP & 0.100 & 0.401 & 1.259 & 1.145 & 1.146 \\
\hline$\Delta[9] \mathrm{CPP}$ & 0.002 & 0.004 & 0.058 & 0.059 & 0.062 \\
\hline [12]CPP & 0.103 & 0.337 & 1.197 & 1.058 & 1.206 \\
\hline$\Delta[12] \mathrm{CPP}$ & 0.001 & 0.005 & 0.020 & 0.021 & 0.065 \\
\hline $\mathrm{F}_{2}[12] \mathrm{CPP}$ & 0.095 & 0.279 & 0.948 & 0.862 & 1.129 \\
\hline$\Delta F_{2}[12] C P P$ & 0.000 & 0.002 & 0.002 & 0.010 & 0.011 \\
\hline 1 & 0.126 & 0.419 & 1.427 & 1.336 & 1.276 \\
\hline$\Delta 1$ & 0.002 & 0.007 & 0.076 & 0.061 & 0.083 \\
\hline 2 & 0.142 & 0.585 & 2.236 & 1.650 & 1.953 \\
\hline$\Delta 2$ & 0.006 & 0.003 & 0.070 & 0.029 & 0.005 \\
\hline 3 & 0.190 & 0.723 & 2.220 & 2.061 & 2.597 \\
\hline$\Delta \mathbf{3}$ & 0.001 & 0.010 & 0.023 & 0.021 & 0.021 \\
\hline 4 & 0.103 & 0.371 & 1.228 & 1.158 & 1.098 \\
\hline$\Delta 4$ & 0.003 & 0.002 & 0.029 & 0.020 & 0.015 \\
\hline
\end{tabular}


Table S5: Affinities of FP-8, CPPs, $p$-quinquephenyl and affinity materials 1-4 towards benzene, toluene, $o$-xylene, $m$ xylene and $p$-xylene.

\begin{tabular}{|c|c|c|c|c|c|}
\hline $\begin{array}{l}\text { Analyte } \\
\text { Affinity } \\
{\left[\mathrm{Hz} \cdot \mathrm{ppm}^{-1}\right]}\end{array}$ & pseudocumene & $\begin{array}{c}\text { 1,2-diethyl- } \\
\text { benzene }\end{array}$ & $\begin{array}{l}\text { 1,3-diethyl- } \\
\text { benzene }\end{array}$ & $\begin{array}{l}\text { 1,4-diethyl- } \\
\text { benzene }\end{array}$ & $\begin{array}{c}\text { 1,3,5-triethyl- } \\
\text { benzene }\end{array}$ \\
\hline FP-8 & 3.79 & 14154.01 & 12737.92 & 8776.99 & 19435.81 \\
\hline$\Delta \mathrm{FP}-8$ & 0.01 & 13.23 & 39.22 & 12.01 & 101.24 \\
\hline p-quinquephenyl & 3.75 & 14886.94 & 13187.54 & 8551.65 & 19917.20 \\
\hline $\begin{array}{l}\Delta p \text { - } \\
\text { quinquephenyl }\end{array}$ & 0.02 & 15.58 & 21.12 & 16.21 & 43.45 \\
\hline [6]CPP & 4.79 & 18581.72 & 16442.97 & 14885.79 & 24167.24 \\
\hline$\Delta[6] \mathrm{CPP}$ & 0.04 & 12.96 & 2.87 & 11.94 & 26.76 \\
\hline [9]CPP & 5.42 & 20471.74 & 15759.57 & 12974.15 & 27319.91 \\
\hline$\Delta[9] \mathrm{CPP}$ & 0.13 & 57.98 & 208.70 & 23.74 & 1521.99 \\
\hline [12]CPP & 5.12 & 16700.53 & 14928.45 & 15050.02 & 23667.45 \\
\hline$\Delta[12] \mathrm{CPP}$ & 0.05 & 41.49 & 11.09 & 25.56 & 48.01 \\
\hline 1 & 5.97 & 21369.95 & 14870.09 & 18450.82 & 31575.84 \\
\hline$\Delta 1$ & 0.11 & 113.86 & 13.53 & 85.50 & 2781.54 \\
\hline 2 & 7.29 & 29164.36 & 23797.38 & 25311.74 & 34522.47 \\
\hline$\Delta 2$ & 0.02 & 7.26 & 3.96 & 19.57 & 68.83 \\
\hline 3 & 7.61 & 29799.13 & 25035.63 & 24379.43 & 35357.21 \\
\hline$\Delta \mathbf{3}$ & 0.02 & 14.32 & 1.09 & 9.04 & 86.08 \\
\hline 4 & 5.46 & 20518.48 & 18589.42 & 18580.00 & 31816.65 \\
\hline$\Delta 4$ & 0.02 & 3.39 & 7.21 & 26.92 & 350.35 \\
\hline
\end{tabular}


Table S6: Affinities of FP-8, CPPs, p-quinquephenyl and affinity materials 1-4 towards cyclohexane, nitrobenzene, 2,4-DNT, TNT and $\mathrm{H}_{2} \mathrm{O}$.

\begin{tabular}{|c|c|c|c|c|c|}
\hline $\begin{array}{l}\text { Affinity } \\
{\left[\mathrm{Hz} \cdot \mathrm{ppm}^{-1}\right]}\end{array}$ & cyclohexane & nitrobenzene & 2,4-DNT & TNT & $\mathrm{H}_{2} \mathrm{O}$ \\
\hline FP-8 & 0.117 & 47.2 & 37948 & 3420715 & 0.964 \\
\hline$\Delta$ FP-8 & 0.002 & 0.6 & 241 & 14614 & 0.009 \\
\hline p-quinquephenyl & 0.118 & 49.9 & 41796 & 3472191 & 1.269 \\
\hline$\Delta p$-quinquephenyl & 0.003 & 2.1 & 271 & 17311 & 0.010 \\
\hline [6]CPP & 0.157 & 60.3 & 42194 & 4027299 & 1.492 \\
\hline$\Delta[6] \mathrm{CPP}$ & 0.001 & 1.5 & 253 & 15640 & 0.009 \\
\hline [9]CPP & 0.153 & 61.7 & 42940 & 4149969 & 1.486 \\
\hline$\Delta[9] \mathrm{CPP}$ & 0.001 & 3.2 & 363 & 16394 & 0.010 \\
\hline [12]CPP & 0.150 & 57.7 & 37511 & 3887612 & 1.389 \\
\hline$\Delta[12] \mathrm{CPP}$ & 0.001 & 1.7 & 348 & 19695 & 0.007 \\
\hline 1 & 0.183 & 68.2 & 46262 & 4737815 & 1.788 \\
\hline$\Delta 1$ & 0.002 & 3.2 & 682 & 29109 & 0.013 \\
\hline 2 & 0.168 & 78.9 & 43925 & 4764808 & 1.532 \\
\hline$\Delta 2$ & 0.004 & 2.8 & 808 & 48272 & 0.010 \\
\hline 3 & 0.162 & 80.3 & 36619 & 4974015 & 1.514 \\
\hline$\Delta \mathbf{3}$ & 0.001 & 0.2 & 550 & 12949 & 0.004 \\
\hline 4 & 0.208 & 72.7 & 45669 & 4903725 & 1.524 \\
\hline$\Delta 4$ & 0.001 & 1.5 & 401 & 12773 & 0.009 \\
\hline
\end{tabular}


Table S7: Affinities of FP-8, CPPs, $p$-quinquephenyl and affinity materials 1-4 towards acetone, GBL, acetophenone, and TATP.

\begin{tabular}{|c|c|c|c|c|}
\hline $\begin{array}{l}\text { Affinity } \\
{\left[\mathrm{Hz} \cdot \mathrm{ppm}^{-1}\right]}\end{array}$ & acetone & GBL & acetophenone & TATP \\
\hline FP-8 & 0.063 & 2.935 & 23.67 & 415.5 \\
\hline$\Delta \mathrm{FP}-8$ & 0.001 & 0.022 & 0.23 & 9.5 \\
\hline$p$-quinquephenyl & 0.063 & 3.523 & 37.96 & 432.8 \\
\hline$\Delta p$-quinquephenyl & 0.002 & 0.020 & 1.85 & 6.6 \\
\hline [6]CPP & 0.070 & 4.505 & 44.63 & 569.8 \\
\hline$\Delta[6] \mathrm{CPP}$ & 0.003 & 0.157 & 1.79 & 5.9 \\
\hline [9]CPP & 0.074 & 4.281 & 43.72 & 541.7 \\
\hline$\Delta[9] \mathrm{CPP}$ & 0.002 & 0.010 & 1.04 & 4.8 \\
\hline [12]CPP & 0.073 & 4.278 & 44.42 & 497.6 \\
\hline$\Delta[12] C P P$ & 0.002 & 0.014 & 0.01 & 4.5 \\
\hline 1 & 0.081 & 5.434 & 60.97 & 528.4 \\
\hline$\Delta 1$ & 0.001 & 0.180 & 0.09 & 19.0 \\
\hline 2 & 0.089 & 6.448 & 65.03 & 653.5 \\
\hline$\Delta 2$ & 0.004 & 0.065 & 5.92 & 1.4 \\
\hline 3 & 0.093 & 6.839 & 72.53 & 620.1 \\
\hline$\Delta \mathbf{3}$ & 0.002 & 0.136 & 0.39 & 5.6 \\
\hline 4 & 0.100 & 6.236 & 53.66 & 646.7 \\
\hline$\Delta \mathbf{4}$ & 0.003 & 0.021 & 0.96 & 26.2 \\
\hline
\end{tabular}




\section{UV/vis Absorption and Emission Spectroscopy}
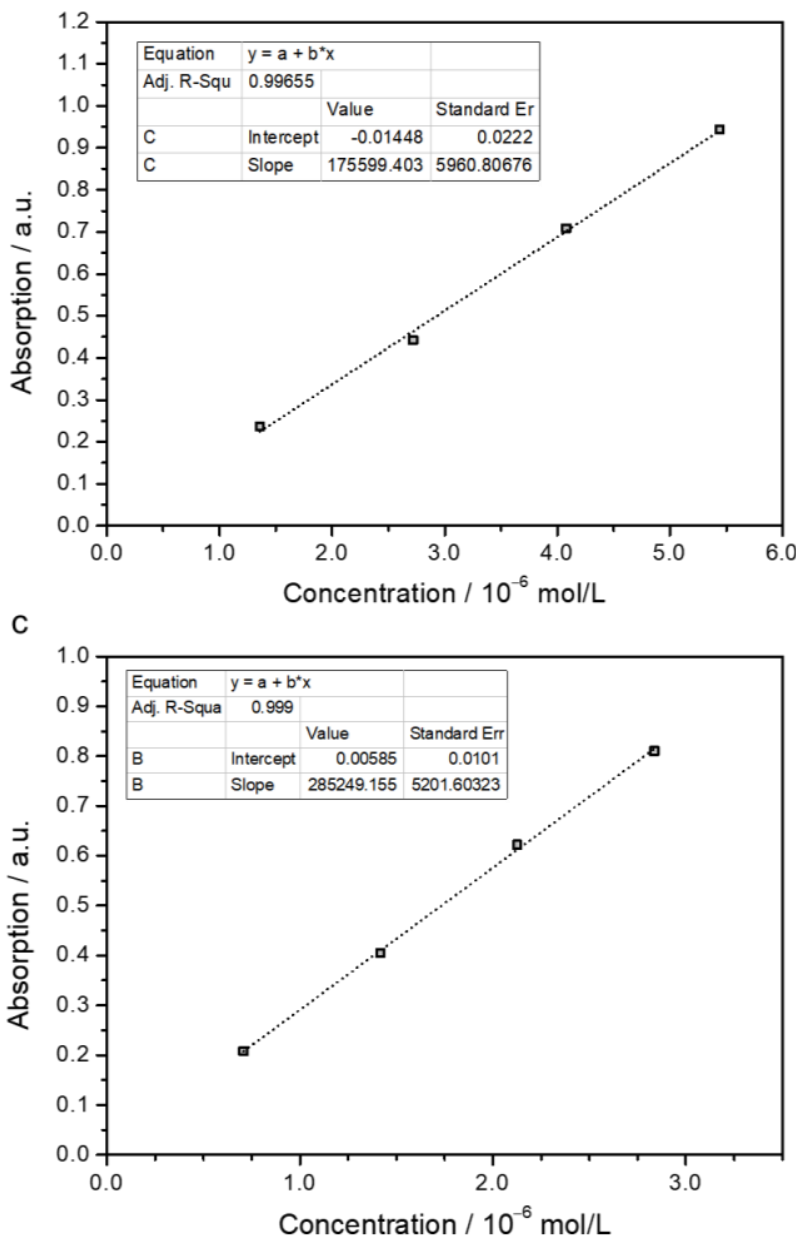

b

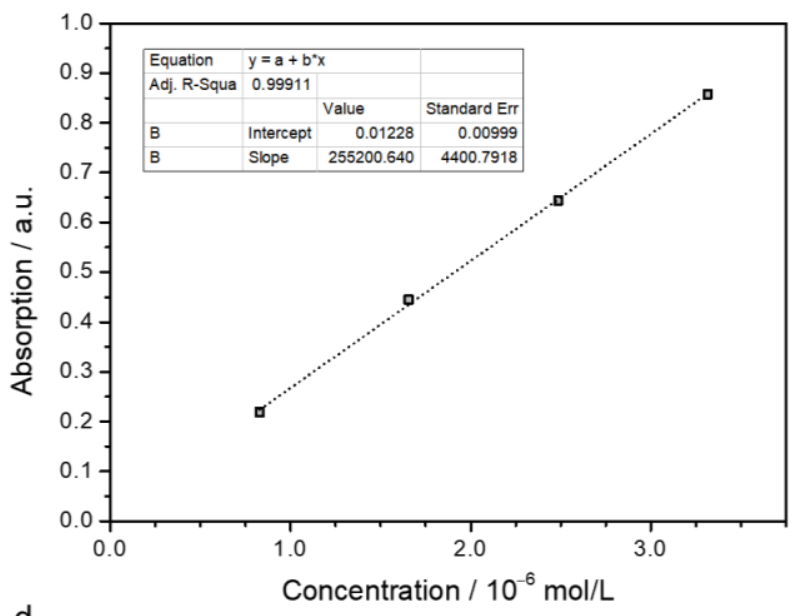

d

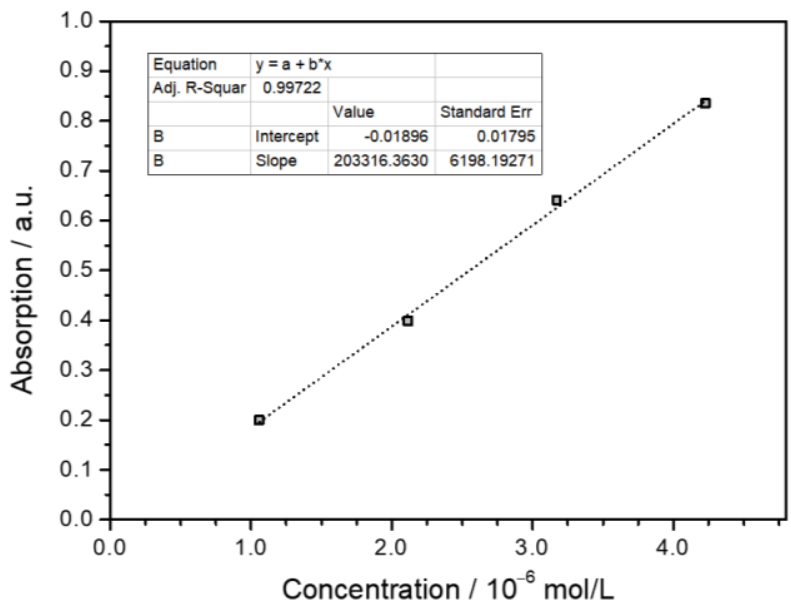

Figure S49: Determination of the extinction coefficient of lemniscate 1 (a), 2 (b), 3 (c), and 4 (d); all measurements were carried out in dichloromethane at room temperature.

\section{Fluorescence quantum yields}

The quantum yields of compounds 1, 2 and 3 were determined at $350 \mathrm{~nm}$ excitation in dichloromethane as described by Jobin Yvon Horiba. ${ }^{[17]}$ Anthracene (ethanol) and quinine sulfate $\left(0.1 \mathrm{M} \mathrm{H}_{2} \mathrm{SO}_{4}\right)$ were used as standards. The integrated fluorescence region for 1 was 440-650 $\mathrm{nm}, 2$ was $400-600 \mathrm{~nm}$ and 3 was $375-600 \mathrm{~nm}$. Quantum yields were calculated using the following equation:

$$
\emptyset_{x}=\emptyset_{S T}\left(\frac{\operatorname{grad}_{x}}{\operatorname{grad}_{S T}}\right)\left(\frac{\eta_{x}^{2}}{\eta_{S T}^{2}}\right)
$$

Where subscripts $x$ and $S T$ denote the unknown and standard respectively, $\phi$ is the quantum yield, grad is the gradient (or slope) from the integrated fluorescence intensity vs. absorbance plot (figure S44), and $\eta$ is the refractive index. The refractive indexes of dichloromethane, $0.1 \mathrm{M} \mathrm{H}_{2} \mathrm{SO}_{4}$ and ethanol used were $1.424,1.332$ and 1.361 respectively. ${ }^{[18]}$ 


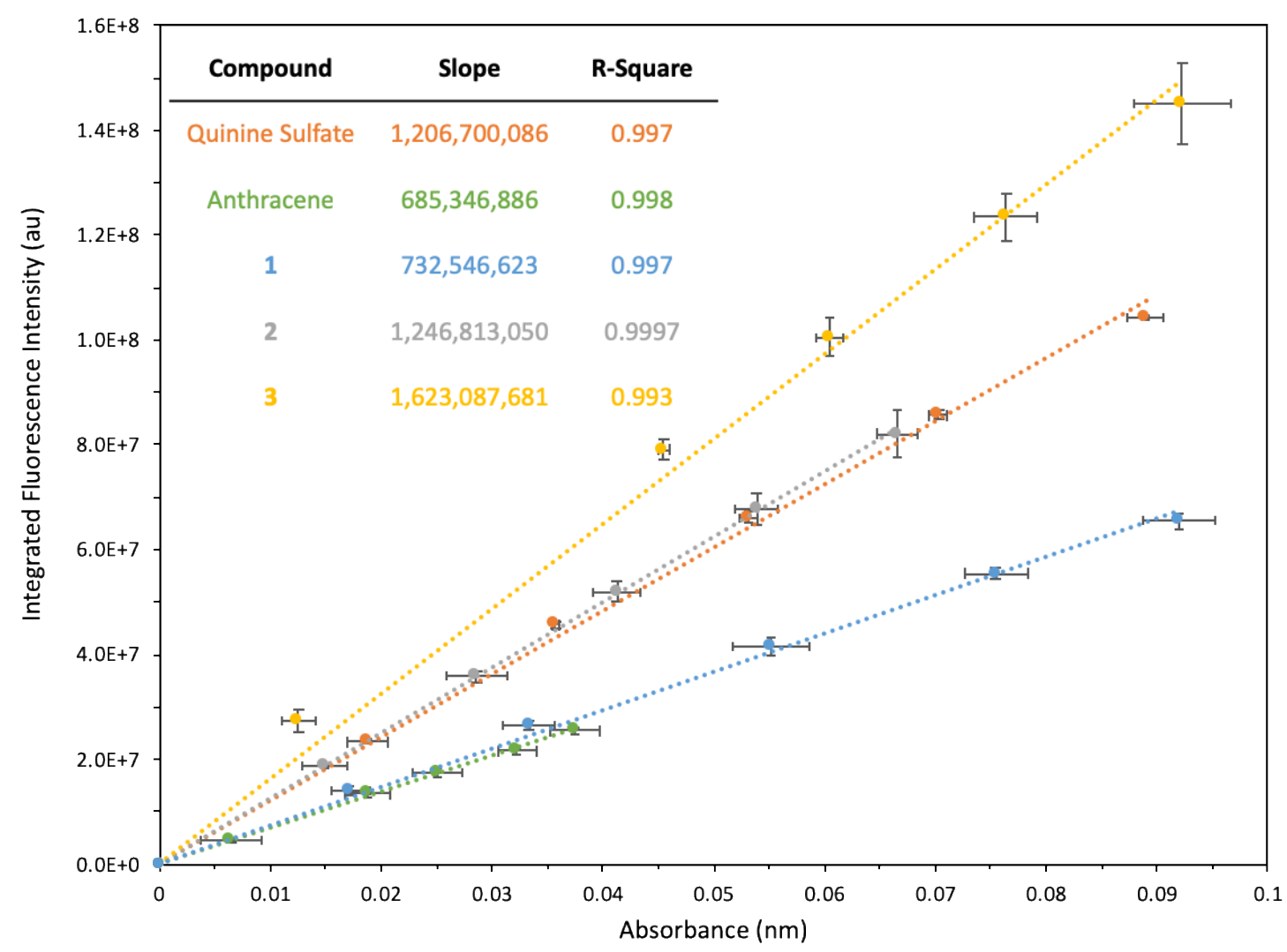

Figure S50: Experimental (circles) and fitted (dashed lines) triplicate data of the integrated fluorescence intensity vs. absorbance of compounds 1 (blue), 2 (grey) and $\mathbf{3}$ (yellow) and standards anthracene (green) and quinine sulfate (orange) used to determine quantum yields.

Table S8: Quantum yields and error of 1-3 with respect to quinine.

\begin{tabular}{cc} 
Sample & $\Phi$ \\
\hline $\mathbf{1}$ & $0.372 \pm 0.003$ \\
$\mathbf{2}$ & $0.639 \pm 0.051$ \\
$\mathbf{3}$ & $0.857 \pm 0.011$
\end{tabular}




\section{Electrochemistry}
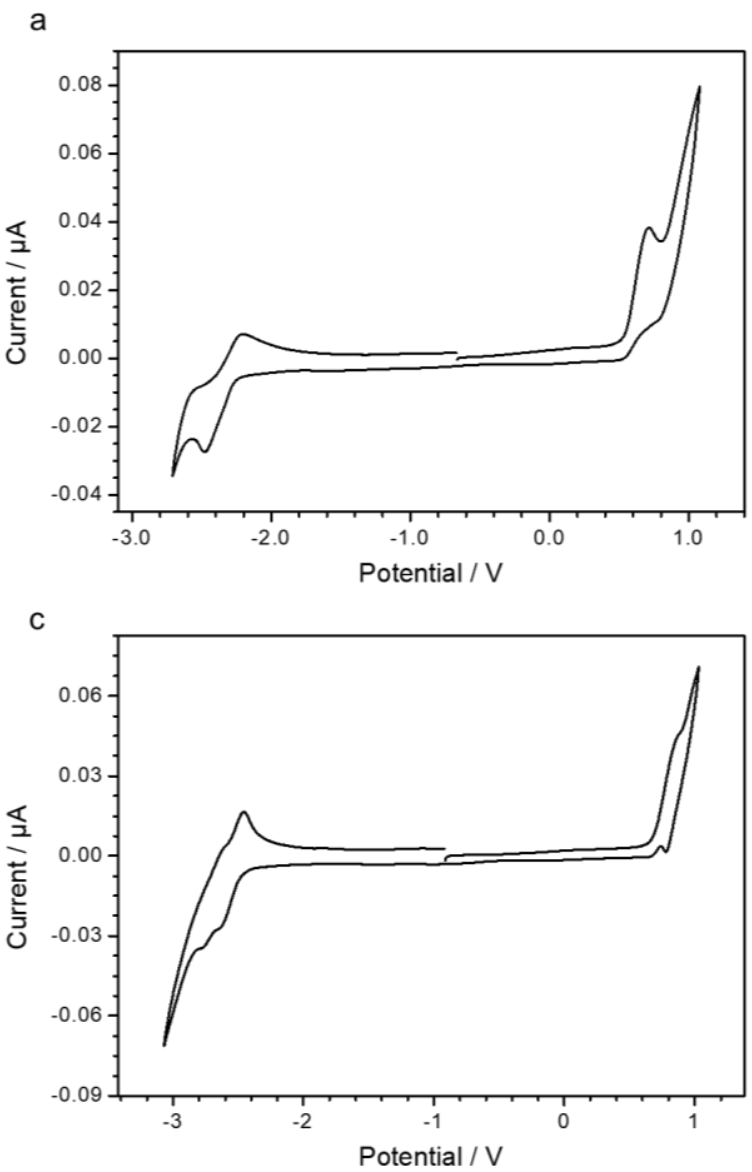

b

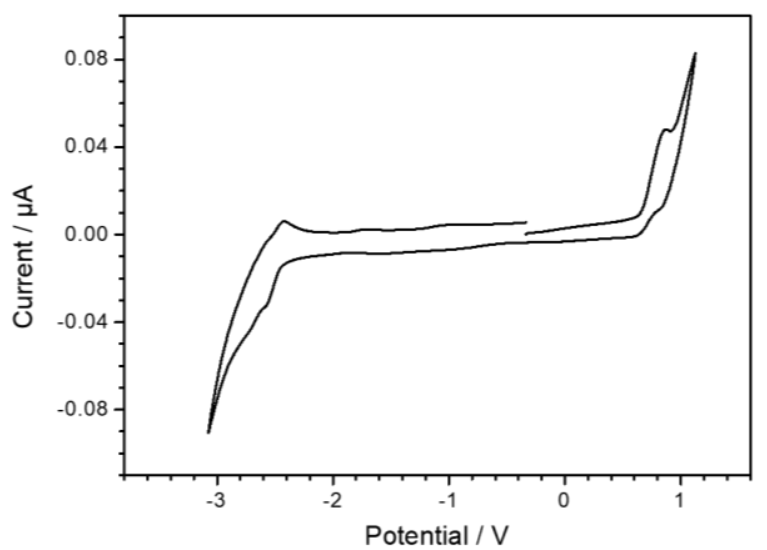

d

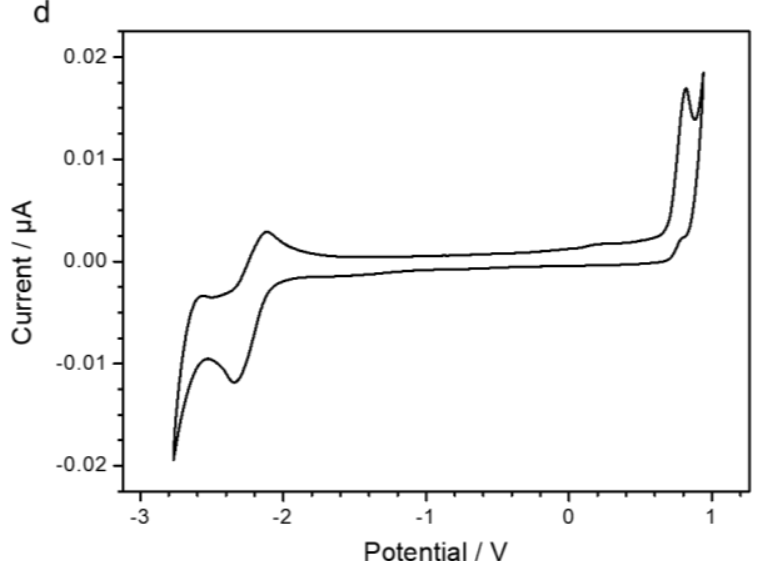

Figure S51: Cyclic voltammograms 1 (a), 2 (b), 3 (c), and 4 (d) vs. Fc/Fc ; Measurements were carried out in THF (0.1 $\mathrm{M} \mathrm{NBu}_{4} \mathrm{NPF}_{6}$, scan rate $200 \mathrm{mV} / \mathrm{s}$ ) at room temperature. 


\section{Computational Details}

\section{General Information}

For consideration of molecular geometries and electronic levels, all structures were fully optimized at TPSS-D3(BJ ${ }^{[19]} /$ def2-TZVP[20] level of theory with default convergence criteria using a $m 4 \mathrm{grid}^{[21]}$ and the Resolution-of-Identity $(\mathrm{RI})^{[22]}$ approximation in TURBOMOLE 7.2 ${ }^{[23]}$ Frequency analysis was carried out for each energy-minimum structure to confirm the nature of the optimized ground state.

\section{Strain energies}

Strain energies were assessed via the homodesmotic reactions ${ }^{[24]}$ shown below.

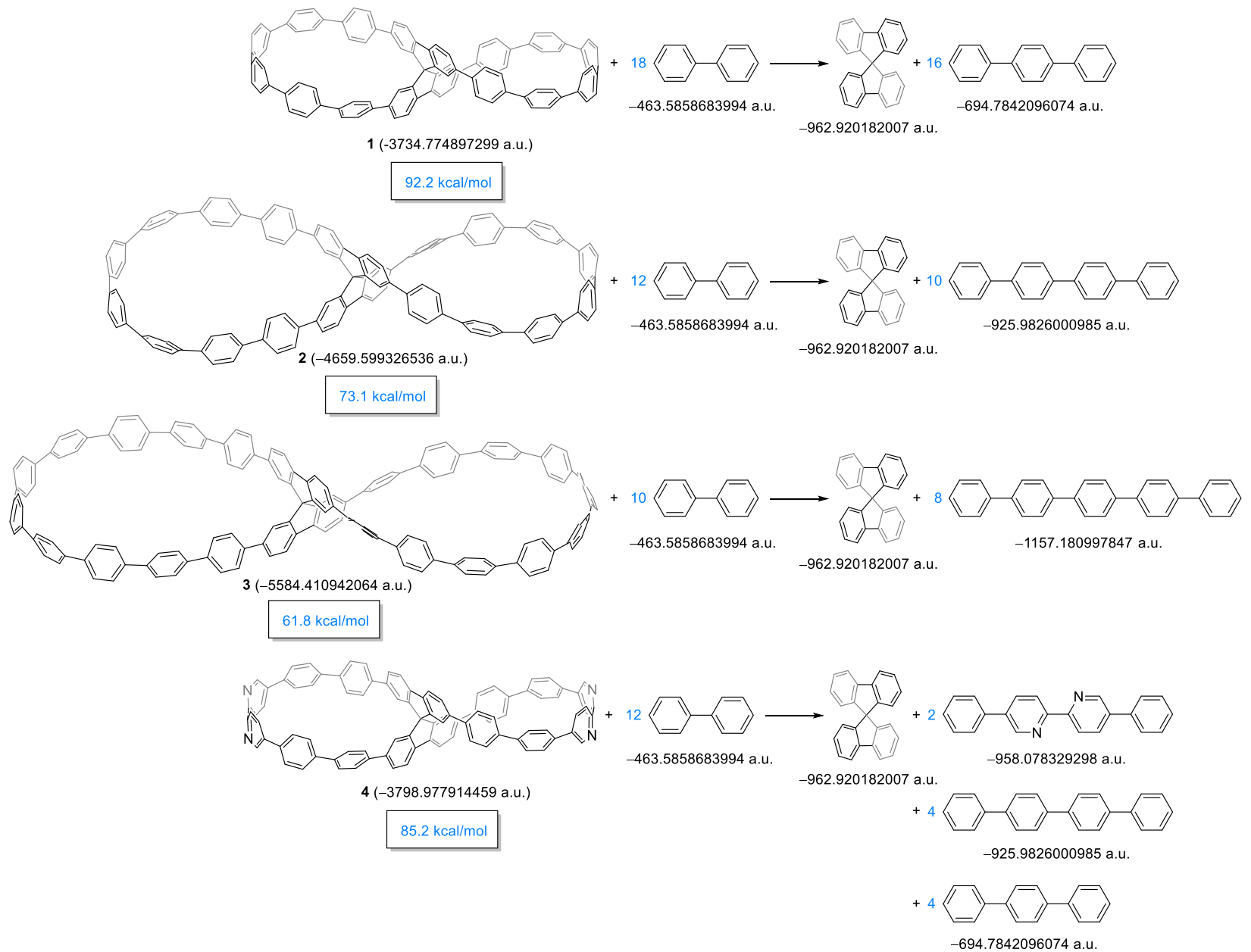

Scheme S1: Homodesmotic strain energy calculation for target compounds 1-4. Enthalpies are calculated at the TPSSD3(BJ)/def2-TZVP level of theory. 


\section{Orbital Picture}

Molecular orbitals and energies are calculated within the Gaussian 09 program suite ${ }^{[25]}$ at the $B 3 L Y P^{[26]} /$ def2-TZVP ${ }^{[27]}$ level of theory employing the conductor-like polarizable continuum model $\left(\mathrm{CPCM}, \mathrm{CH}_{2} \mathrm{Cl}_{2}\right) \cdot{ }^{[28]}$ For these calculations the geometry optimized structures were used (see above, TPSS-D3(BJ)/def2-TZVP) (Fig. S52).

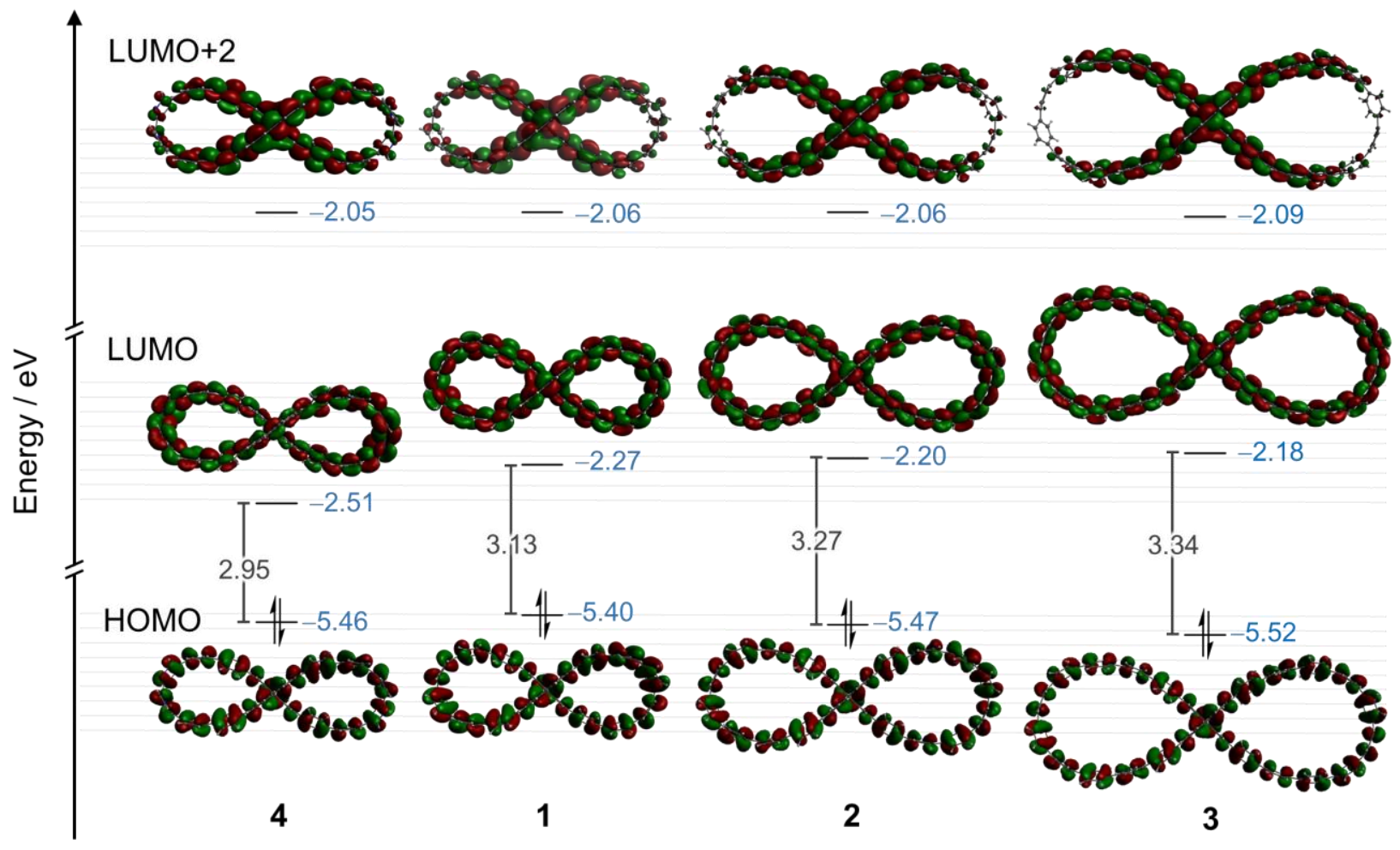

Figure S52: Kohn-Sham Molecular orbitals of target compounds 1-4 together with the orbital energy levels (isosurface 0.01 a.u.).

\section{Electronic Transitions}

UV/vis spectra of 1-4 were calculated using the geometry optimized structures and excited-state calculations were performed within the time-dependent (TD) ${ }^{[28,29]}$ DFT approximation employing the conductor-like polarizable continuum model, $\operatorname{CPCM}\left(\mathrm{CH}_{2} \mathrm{Cl}_{2}\right)$ with 12 roots. Geometry optimization of the first excited state $\mathrm{S} 1$ at $B 3 L Y P / d e f 2-T Z V P$ level appeared to be computationally very demanding even for the smallest derivative 1 . Hence, we here compare only results obtained for electronic excitations. The results for the major electronic transitions can be found in Table S9-S12.

In the experiment, the fluorescence of compound $\mathbf{4}$ in solution is completely quenched (see Fig. 3a) in contradiction to recent observations which reported that embedded nitrogen atoms have little impact on the electronic structure. ${ }^{[30]}$ A detailed investigation of the apparently different excited state relaxation mechanisms of compound $\mathbf{1}$ and $\mathbf{4}$, however, exceeds the scope of this study. 
Table S9: Electronic transitions of 1 calculated at the TD-B3LYP/def2-TZVP level of theory employing $\mathrm{CPCM}\left(\mathrm{CH}_{2} \mathrm{Cl}_{2}\right)$ with 12 roots; Major transitions are highlighted grey.

\begin{tabular}{|c|c|c|c|c|}
\hline No. & $\begin{array}{c}\text { Energy } \\
\left(\mathrm{cm}^{-1}\right)\end{array}$ & $\begin{array}{l}\text { Wavelength } \\
\quad(\mathrm{nm})\end{array}$ & $\begin{array}{l}\text { Osc. Strength } \\
\text { (a.u.) }\end{array}$ & $\begin{array}{c}\text { Major } \\
\text { contributions }\end{array}$ \\
\hline 1 & 21567 & 463.7 & 0.027 & $\begin{array}{c}\mathrm{H}-1 \rightarrow \mathrm{L}+1(13 \%) \\
\mathrm{HOMO} \rightarrow \mathrm{LUMO}(82 \%)\end{array}$ \\
\hline 2 & 22591 & 442.7 & 0.051 & $\begin{array}{l}\mathrm{H}-1 \rightarrow \text { LUMO }(51 \%) \\
\mathrm{HOMO} \rightarrow \mathrm{L}+1(44 \%)\end{array}$ \\
\hline 3 & 23868 & 419.0 & 3.242 & $\begin{array}{l}\mathrm{H}-2 \rightarrow \mathrm{LUMO}(14 \%) \\
\mathrm{HOMO} \rightarrow \mathrm{L}+2(80 \%)\end{array}$ \\
\hline 4 & 24431 & 409.3 & 0.622 & $\begin{array}{l}\text { H-2 } \rightarrow \text { LUMO }(82 \%) \\
\text { HOMO } \rightarrow \text { L+2 (15\%) }\end{array}$ \\
\hline 5 & 24846 & 402.5 & 0.000 & $\begin{array}{c}\mathrm{H}-1 \rightarrow \mathrm{LUMO}(45 \%) \\
\mathrm{HOMO} \rightarrow \mathrm{L}+1(52 \%) \\
\mathrm{H}-2 \rightarrow \mathrm{L}+2(27 \%)\end{array}$ \\
\hline 6 & 24957 & 400.7 & 0.228 & $\begin{array}{c}\mathrm{H}-1 \rightarrow \mathrm{L}+1(44 \%) \\
\mathrm{HOMO} \rightarrow \mathrm{LUMO}(13 \%)\end{array}$ \\
\hline 7 & 25732 & 388.6 & 0.000 & $\begin{array}{c}H-2 \rightarrow L+1(14 \%) \\
H-1 \rightarrow L+2(82 \%)\end{array}$ \\
\hline 8 & 26085 & 383.4 & 1.151 & $\begin{array}{l}H-2 \rightarrow L+2(68 \%) \\
H-1 \rightarrow L+1(27 \%)\end{array}$ \\
\hline 9 & 26441 & 378.2 & 0.000 & $\begin{array}{l}H-2 \rightarrow L+1(82 \%) \\
H-1 \rightarrow L+2(15 \%)\end{array}$ \\
\hline 10 & 26693 & 37.5 & 0.299 & $\begin{array}{c}\mathrm{H}-3 \rightarrow \text { LUMO }(81 \%) \\
\mathrm{H}-1 \rightarrow \mathrm{L}+1(11 \%)\end{array}$ \\
\hline 11 & 27199 & 367.7 & 0.219 & $\mathrm{HOMO} \rightarrow \mathrm{L}+3(89 \%)$ \\
\hline 12 & 27775 & 360.0 & 0.007 & $\begin{array}{l}H-3 \rightarrow L+2(75 \%) \\
H-2 \rightarrow L+3(12 \%)\end{array}$ \\
\hline
\end{tabular}

Table S10: Electronic transitions of 2 calculated at the TD-B3LYP/def2-TZVP level of theory employing $C P C M\left(\mathrm{CH}_{2} \mathrm{Cl}_{2}\right)$ with 12 roots; Major transitions are highlighted grey.

\begin{tabular}{|c|c|c|c|c|}
\hline No. & $\begin{array}{l}\text { Energy } \\
\left(\mathrm{cm}^{-1}\right)\end{array}$ & $\begin{array}{l}\text { Wavelength } \\
(\mathrm{nm})\end{array}$ & $\begin{array}{l}\text { Osc. Strength } \\
\text { (a.u.) }\end{array}$ & $\begin{array}{c}\text { Major } \\
\text { contributions }\end{array}$ \\
\hline 1 & 22833 & 438.0 & 0.047 & $\begin{array}{c}\mathrm{H}-1 \rightarrow \mathrm{L}+1(21 \%) \\
\mathrm{HOMO} \rightarrow \mathrm{LUMO}(68 \%)\end{array}$ \\
\hline 2 & 23401 & 427.3 & 0.048 & $\begin{array}{l}\mathrm{H}-1 \rightarrow \mathrm{LUMO}(46 \%) \\
\mathrm{HOMO} \rightarrow \mathrm{L}+1(44 \%)\end{array}$ \\
\hline 3 & 24360 & 410.5 & 4.872 & $\begin{array}{l}\mathrm{H}-2 \rightarrow \text { LUMO }(27 \%) \\
\mathrm{HOMO} \rightarrow \mathrm{L}+2(60 \%)\end{array}$ \\
\hline 4 & 25290 & 395.4 & 0.235 & $\begin{array}{l}\text { H-2 } \rightarrow \text { LUMO }(64 \%) \\
\text { HOMO } \rightarrow \text { L+2 (30\%) }\end{array}$ \\
\hline 5 & 25439 & 393.1 & 0.576 & $\begin{array}{c}\mathrm{H}-3 \rightarrow \text { LUMO }(19 \%) \\
\mathrm{H}-2 \rightarrow \mathrm{L}+2(14 \%) \\
\mathrm{H}-1 \rightarrow \mathrm{L}+1(34 \%) \\
\mathrm{HOMO} \rightarrow \mathrm{LUMO}(19 \%) \\
\mathrm{HOMO} \rightarrow \mathrm{L}+3(12 \%)\end{array}$ \\
\hline 6 & 25863 & 386.7 & 0.000 & $\begin{array}{l}\mathrm{H}-1 \rightarrow \mathrm{LUMO}(46 \%) \\
\mathrm{HOMO} \rightarrow \mathrm{L}+1(50 \%)\end{array}$ \\
\hline
\end{tabular}




\begin{tabular}{|c|c|c|c|c|}
\hline 7 & 26019 & 384.3 & 1.565 & $\begin{array}{l}H-2 \rightarrow L+2(74 \%) \\
H-1 \rightarrow L+1(10 \%)\end{array}$ \\
\hline 8 & 26244 & 381.0 & 0.000 & $\mathrm{H}-1 \rightarrow \mathrm{L}+2(88 \%)$ \\
\hline 9 & 26459 & 377.9 & 0.000 & $\begin{array}{c}\mathrm{H}-4 \rightarrow \mathrm{LUMO}(12 \%) \\
\mathrm{H}-2 \rightarrow \mathrm{L}+1(85 \%)\end{array}$ \\
\hline 10 & 27019 & 370.1 & 0.123 & $\begin{array}{c}\mathrm{H}-3 \rightarrow \text { LUMO }(47 \%) \\
\mathrm{H}-1 \rightarrow \mathrm{L}+1(30 \%)\end{array}$ \\
\hline 11 & 27487 & 363.8 & 0.059 & $\begin{array}{c}\mathrm{H}-3 \rightarrow \mathrm{LUMO}(23 \%) \\
\mathrm{HOMO} \rightarrow \mathrm{L}+3(72 \%) \\
\mathrm{H}-3 \rightarrow \mathrm{L}+2(66 \%)\end{array}$ \\
\hline 12 & 27548 & 363.0 & 0.008 & $\begin{array}{l}H-2 \rightarrow L+3(11 \%) \\
H-1 \rightarrow L+4(11 \%)\end{array}$ \\
\hline
\end{tabular}

Table S11: Electronic transitions of 3 calculated at the TD-B3LYP/def2-TZVP level of theory employing $C P C M\left(\mathrm{CH}_{2} \mathrm{Cl}_{2}\right)$ with 12 roots; Major transitions are highlighted grey.

\begin{tabular}{|c|c|c|c|c|}
\hline No. & $\begin{array}{l}\text { Energy } \\
\left(\mathrm{cm}^{-1}\right)\end{array}$ & $\begin{array}{l}\text { Wavelength } \\
\quad(\mathrm{nm})\end{array}$ & $\begin{array}{l}\text { Osc. Strength } \\
\text { (a.u.) }\end{array}$ & $\begin{array}{c}\text { Major } \\
\text { contributions }\end{array}$ \\
\hline \multirow{3}{*}{1} & \multirow{3}{*}{23492} & \multirow{3}{*}{425.7} & \multirow{3}{*}{0.012} & $H-2 \rightarrow L+2(11 \%)$ \\
\hline & & & & $\mathrm{H}-1 \rightarrow \mathrm{L}+1(21 \%)$ \\
\hline & & & & HOMO $\rightarrow$ LUMO (58\%) \\
\hline \multirow{2}{*}{2} & \multirow{2}{*}{23982} & \multirow{2}{*}{417.0} & \multirow{2}{*}{0.016} & $\mathrm{H}-1 \rightarrow$ LUMO $(41 \%)$ \\
\hline & & & & $\mathrm{HOMO} \rightarrow \mathrm{L}+1(38 \%)$ \\
\hline \multirow{2}{*}{3} & \multirow{2}{*}{24385} & \multirow{2}{*}{410.1} & \multirow{2}{*}{5.645} & $\mathrm{H}-2 \rightarrow$ LUMO $(26 \%)$ \\
\hline & & & & $\mathrm{HOMO} \rightarrow \mathrm{L}+2(55 \%)$ \\
\hline \multirow{4}{*}{4} & \multirow{4}{*}{25312} & \multirow{4}{*}{395.1} & \multirow{4}{*}{1.731} & $\mathrm{H}-3 \rightarrow$ LUMO (35\%) \\
\hline & & & & $\mathrm{H}-1 \rightarrow \mathrm{L}+1(24 \%)$ \\
\hline & & & & HOMO $\rightarrow$ LUMO (12\%) \\
\hline & & & & $\mathrm{HOMO} \rightarrow \mathrm{L}+3(24 \%)$ \\
\hline \multirow{2}{*}{5} & \multirow{2}{*}{25455} & \multirow{2}{*}{392.9} & \multirow{2}{*}{0.306} & $\mathrm{H}-2 \rightarrow$ LUMO $(59 \%)$ \\
\hline & & & & $\mathrm{HOMO} \rightarrow \mathrm{L}+2(27 \%)$ \\
\hline 6 & 25723 & 388.8 & 1.302 & $\mathrm{H}-2 \rightarrow \mathrm{L}+2(80 \%)$ \\
\hline \multirow{4}{*}{7} & \multirow{4}{*}{26199} & \multirow{4}{*}{381.7} & \multirow{4}{*}{0.000} & $\mathrm{H}-4 \rightarrow$ LUMO (11\%) \\
\hline & & & & $H-2 \rightarrow L+1(22 \%)$ \\
\hline & & & & $\mathrm{H}-1 \rightarrow \mathrm{L}+2(47 \%)$ \\
\hline & & & & $\mathrm{HOMO} \rightarrow \mathrm{L}+4(14 \%)$ \\
\hline \multirow{2}{*}{8} & \multirow{2}{*}{26421} & \multirow{2}{*}{378.5} & \multirow{2}{*}{0.000} & $\mathrm{H}-1 \rightarrow$ LUMO $(41 \%)$ \\
\hline & & & & $\mathrm{HOMO} \rightarrow \mathrm{L}+1(51 \%)$ \\
\hline \multirow[t]{3}{*}{9} & \multirow{3}{*}{26707} & \multirow{3}{*}{374.4} & \multirow{3}{*}{0.000} & $\mathrm{H}-2 \rightarrow \mathrm{L}+1(54 \%)$ \\
\hline & & & & $\mathrm{H}-1 \rightarrow \mathrm{L}+2(36 \%)$ \\
\hline & & & & $\mathrm{H}-3 \rightarrow$ LUMO $(32 \%)$ \\
\hline 10 & 27141 & 368.4 & 0.003 & $\mathrm{H}-1 \rightarrow \mathrm{L}+1(36 \%)$ \\
\hline & & & & HOMO $\rightarrow$ LUMO $(18 \%)$ \\
\hline 11 & 27150 & 368.3 & 0.080 & $\mathrm{H}-3 \rightarrow \mathrm{L}+2(70 \%)$ \\
\hline & & & & $H-1 \rightarrow L+4(11 \%)$ \\
\hline 12 & 27321 & 366.0 & 0.022 & $\mathrm{H}-3 \rightarrow$ LUMO (26\%) \\
\hline & 2601 & 300.0 & 0.022 & $\mathrm{HOMO} \rightarrow \mathrm{L}+3(66 \%)$ \\
\hline
\end{tabular}


Table S12: Electronic transitions of 4 calculated at the TD-B3LYP/def2-TZVP level of theory employing $C P C M\left(\mathrm{CH}_{2} \mathrm{Cl}_{2}\right)$ with 12 roots; Major transitions are highlighted grey.

\begin{tabular}{|c|c|c|c|c|}
\hline No. & $\begin{array}{l}\text { Energy } \\
\left(\mathrm{cm}^{-1}\right)\end{array}$ & $\begin{array}{l}\text { Wavelength } \\
\quad(\mathrm{nm})\end{array}$ & $\begin{array}{l}\text { Osc. Strength } \\
\text { (a.u.) }\end{array}$ & $\begin{array}{c}\text { Major } \\
\text { contributions }\end{array}$ \\
\hline 1 & 20315 & 492.2 & 0.131 & $\begin{array}{c}\mathrm{H}-1 \rightarrow \mathrm{L}+1(18 \%) \\
\mathrm{HOMO} \rightarrow \mathrm{LUMO}(78 \%)\end{array}$ \\
\hline 2 & 20953 & 477.3 & 0.077 & $\begin{array}{l}\mathrm{H}-1 \rightarrow \mathrm{LUMO}(37 \%) \\
\mathrm{HOMO} \rightarrow \mathrm{L}+1(60 \%)\end{array}$ \\
\hline 3 & 23243 & 430.2 & 2.123 & H-2 $\rightarrow$ LUMO (94\%) \\
\hline 4 & 23409 & 427.2 & 0.004 & $\begin{array}{l}\mathrm{H}-1 \rightarrow \mathrm{LUMO}(58 \%) \\
\mathrm{HOMO} \rightarrow \mathrm{L}+1(39 \%)\end{array}$ \\
\hline 5 & 23671 & 422.5 & 0.336 & $\begin{array}{c}\mathrm{H}-1 \rightarrow \mathrm{L}+1(70 \%) \\
\mathrm{HOMO} \rightarrow \mathrm{LUMO}(19 \%)\end{array}$ \\
\hline 6 & 24226 & 412.8 & 0.000 & $\mathrm{H}-2 \rightarrow \mathrm{L}+1(93 \%)$ \\
\hline 7 & 24483 & 408.4 & 1.711 & $\mathrm{HOMO} \rightarrow \mathrm{L}+2(94 \%)$ \\
\hline 8 & 25881 & 386.4 & 0.217 & H-3 $\rightarrow$ LUMO (73\%) \\
\hline 9 & 26290 & 380.4 & 0.785 & $\begin{array}{c}\mathrm{H}-2 \rightarrow \mathrm{L}+2(33 \%) \\
\mathrm{HOMO} \rightarrow \mathrm{L}+3(60 \%)\end{array}$ \\
\hline 10 & 26411 & 378.6 & 0.189 & $\begin{array}{c}\mathrm{H}-3 \rightarrow \mathrm{LUMO}(17 \%) \\
\mathrm{H}-2 \rightarrow \mathrm{L}+2(55 \%) \\
\mathrm{HOMO} \rightarrow \mathrm{L}+3(26 \%)\end{array}$ \\
\hline 11 & 26624 & 375.6 & 0.009 & $\mathrm{H}-3 \rightarrow \mathrm{L}+1(92 \%)$ \\
\hline 12 & 26638 & 375.4 & 0.000 & $\begin{array}{c}\mathrm{H}-4 \rightarrow \mathrm{LUMO}(13 \%) \\
\mathrm{H}-1 \rightarrow \mathrm{L}+2(76 \%)\end{array}$ \\
\hline
\end{tabular}

\section{Molecular Analyte/Host Model Systems}

To investigate the influence of the analyte size on the analyte/host interactions, 28 alkyl-substituted benzenes of varying volume were docked into single host molecules ([9]CPP, [12]CPP, and 1-3) applying the intermolecular force field $x T B-I F F$. Solvation contributions were considered by the implicit Generalized Born and Surface Area continuum solvation model GBSA as implemented in $x t b 6.1,{ }^{[31]}$ using THF as solvent. The energetically lowest structures of the docked host-guest complexes were further optimized at TPSS-D3(BJ)/def2-TZVPlevel of theory in TURBOMOLE 7.2 with default convergence criteria, Resolution-of-Identity (RI) approximation and $m 4$ grid. Interaction energies were calculated as the difference between the host-guest complex and the individual host and guest structures. SASAs were predicted with UCSF Chimera ${ }^{[32]}$ and are summarized in Table S13-S16.

Table S13: Examined alkyl substituted phenylenes with their corresponding SASA in $\AA^{2}$ (sorted by SASA).

\begin{tabular}{lc} 
Guest & SASA / $\AA^{2}$ \\
\hline benzene & 227.0 \\
methylbenzene & 255.3 \\
1,2-dimethylbenzene & 277.4 \\
ethylbenzene & 282.3 \\
1,3-dimethylbenzene & 283.5
\end{tabular}


1,4-dimethylbenzene $\quad 283.7$

1,2,3-trimethylbenzene $\quad 297.8$

i-propylbenzene $\quad 304.3$

1,3,5-trimethylbenzene $\quad 311.7$

t-butylbenzene $\quad 318.8$

1,2-diethylbenzene $\quad 325.3$

1,2,3,5-tetramethylbenzene $\quad 326.2$

1,4-diethylbenzene $\quad 334.0$

1,3-diethylbenzene $\quad 335.5$

1,2,3,4,5-pentamethylbenzene $\quad 341.6$

1,2,3,4,5-hexamethylbenzene $\quad 356.3$

1,2-di-i-propylbenzene $\quad 361.7$

1,3-di-i-propylbenzene $\quad 381.6$

1,4-di-i-propylbenzene $\quad 381.9$

1,3,5-triethylbenzene $\quad 388.8$

1,3-di-t-butylbenzene $\quad 408.2$

1,4-di-t-butylbenzene $\quad 411.0$

1,3,5-triethyl-2,4,6-trimethylbenzene $\quad 419.2$

1,2,3-triethyl-4,5,6-trimethylbenzene $\quad 420.9$

1,3,5-tri-i-Propylbenzene $\quad 457.1$

1,3,5-tri-i-propyl-2,4,6-trimethylbenzene $\quad 478.6$

1,3,5-tri-t-butylbenzene $\quad 495.8$

1,3,5-ethyl-2,4,6-tri-i-propylbenzene $\quad 516.8$

Table S14: Absolute energy in Hartree after optimization on TPSS-D3(BJ)/def2-TZVP level of different host and guest molecules.

\begin{tabular}{lcc} 
Molecule & Absolute Energy / Hartree & SASA / $\AA^{2}$ \\
\hline benzene & -232.3880325 & 227.0 \\
methylbenzene & -271.7288095 & 255.3 \\
ethylbenzene & -311.0659982 & 282.3 \\
i-propylbenzene & -350.4035675 & 304.3 \\
$t$-butylbenzene & -389.7398786 & 318.8 \\
1,2-dimethylbenzene & -311.068155 & 277.4 \\
1,2-diethylbenzene & -389.7415687 & 325.3 \\
1,2-di-i-propylbenzene & -468.4137871 & 361.7 \\
1,3-dimethylbenzene & -311.0695259 & 283.5 \\
1,3-diethylbenzene & -389.7426382 & 335.5 \\
1,3-di-i-propylbenzene & -468.4193072 & 381.6 \\
1,3-di-t-butylbenzene & -547.0921735 & 408.2 \\
1,4-dimethylbenzene & -311.069463 & 283.7 \\
1,4-diethylbenzene & -389.7409555 & 334.0 \\
1,4-di-i-propylbenzene & -468.4190432 & 381.9 \\
1,4-di-t-butylbenzene & -547.0918571 & 411.0 \\
1,2,3-trimethylbenzene & -350.4090535 & 297.8 \\
1,3,5-trimethylbenzene & -350.4103171 & 311.7 \\
1,3,5-triethylbenzene & -468.4192925 & 388.8
\end{tabular}




\begin{tabular}{lll} 
1,3,5-tri-i-propylbenzene & -586.4352576 & 457.1 \\
1,3,5-tri-t-butylbenzene & -704.4449126 & 495.8 \\
1,2,3,5-tetramethylbenzene & -389.7497722 & 326.2 \\
1,2,3,4,5-pentamethylbenzene & -429.0867816 & 341.6 \\
$\begin{array}{l}\text { 1,2,3,4,5,6-hexamethylbenzene } \\
\text { 1,2,3-triethyl-4,5,6- }\end{array}$ & -468.4188567 & 356.3 \\
trimethylbenzene & -586.4323311 & 420.9 \\
1,3,5-triethyl-2,4,6- & -586.4314026 & 419.2 \\
trimethylbenzene & & 478.6 \\
1,3,5-tri-i-propyl-2,4,6- & -704.4209894 & 516.8 \\
trimethylbenzene & -822.4224604 & \\
propylbenzene & -2080.688481 & \\
\hdashline-- - & -2774.307094 & \\
[9]CPP & -3734.776371 & \\
[12]CPP & -4659.595525 & \\
$\mathbf{2}$ & -5584.406583 & \\
$\mathbf{3}$ & & \\
\hline
\end{tabular}

Table S15: Absolute energy in Hartree after optimization on TPSS-D3(BJ)/def2-TZVP level of different singly filled hostguest structures.

\begin{tabular}{|c|c|c|c|c|c|}
\hline Guest & [9]CPP & {$[12] C P P$} & 1 & 2 & 3 \\
\hline benzene & -2313.09566 & -3006.71021 & -3967.18677 & -4892.00283 & -5816.81075 \\
\hline methylbenzene & -2352.43954 & -3046.05228 & -4006.52892 & -4931.34616 & -5856.15436 \\
\hline ethylbenzene & -2391.77863 & -3085.39237 & -4045.86988 & -4970.68623 & -5895.49163 \\
\hline i-propylbenzene & -2431.11870 & -3124.73172 & -4085.20811 & -5010.02589 & -5934.83537 \\
\hline t-butylbenzene & -2470.45955 & -3164.06718 & -4124.54819 & - & -5974.17061 \\
\hline 1,2-dimethylbenzene & -2391.78350 & -3085.39588 & -4045.87448 & -4970.68872 & -5895.49639 \\
\hline 1,2-diethylbenzene & -2470.46245 & -3164.06954 & -4124.55114 & -5049.36382 & -5974.17134 \\
\hline 1,2-di-i-propylbenzene & -2549.13788 & -3242.74293 & -4203.22114 & -5128.03965 & -6052.84975 \\
\hline 1,3-dimethylbenzene & -2391.78279 & -3085.39576 & -4045.87422 & -4970.68823 & -5895.49996 \\
\hline 1,3-diethylbenzene & -2470.46239 & -3164.07317 & -4124.54845 & -5049.36791 & -5974.17498 \\
\hline 1,3-di-i-propylbenzene & -2549.13734 & -3242.75247 & -4203.23136 & -5128.04347 & -6052.85177 \\
\hline 1,3-di-t-butylbenzene & -2627.81432 & -3321.42302 & -4281.90095 & -5206.72569 & -6131.52759 \\
\hline 1,4-dimethylbenzene & -2391.78297 & -3085.39520 & -4045.87294 & -4970.68911 & -5895.49804 \\
\hline 1,4-diethylbenzene & -2470.46227 & -3164.07339 & -4124.54841 & -5049.36939 & -5974.17203 \\
\hline 1,4-di-i-propylbenzene & -2549.13586 & -3242.75069 & -4203.22835 & -5128.04993 & -6052.85244 \\
\hline 1,4-di-t-butylbenzene & -2627.81000 & -3321.42534 & -4281.90408 & -5206.72392 & -6131.52818 \\
\hline 1,2,3-trimethylbenzene & -2431.12568 & -3124.73698 & -4085.21370 & -5010.03027 & -5934.83882 \\
\hline 1,3,5-trimethylbenzene & -2431.12497 & -3124.73856 & -4085.21608 & -5010.03068 & -5934.84220 \\
\hline 1,3,5-triethylbenzene & -2549.14383 & -3242.75371 & -4203.23340 & -5128.04656 & -6052.85802 \\
\hline 1,3,5-tri-i-propylbenzene & -2667.15876 & -3360.76954 & -4321.24987 & -5246.06406 & -6170.87256 \\
\hline 1,3,5-tri-t-butylbenzene & -2785.16668 & -3478.78005 & -4439.25128 & -5364.07641 & -6288.88347 \\
\hline 1,2,3,5-tetramethylbenzene & -2470.46815 & -3164.07960 & -4124.55983 & -5049.37252 & -5974.17883 \\
\hline 1,2,3,4,5-pentamethylbenzene & -2509.80652 & -3203.41600 & -4163.89754 & -5088.71193 & -6013.52070 \\
\hline
\end{tabular}




\begin{tabular}{|c|c|c|c|c|c|}
\hline $\begin{array}{l}\text { 1,2,3,4,5,6-hexa-methylbenzene } \\
1,2,3 \text {-triethyl-4,5,6- }\end{array}$ & -2549.14104 & -3242.74946 & -4203.23089 & -5128.04680 & -6052.85715 \\
\hline trimethylbenzene & -2667.15619 & -3360.76847 & -4321.24017 & -5246.06310 & -6170.86731 \\
\hline $\begin{array}{l}\text { 1,3,5-triethyl-2,4,6- } \\
\text { trimethylbenzene } \\
\text { 1,3,5-tri-i-propyl-2,4,6- }\end{array}$ & -2667.15398 & -3360.76578 & -4321.24687 & -5246.06467 & -6170.86998 \\
\hline $\begin{array}{l}\text {,3,3-tri-I-propyl-2,4,6- } \\
\text { trimethylbenzene } \\
\text { 1,3,5-triethyl-2,4,6-tri-i- }\end{array}$ & -2785.14916 & -3478.75770 & -4439.23432 & -5364.05569 & -6288.86298 \\
\hline opylbenzene & -2903.15715 & -3596.76152 & -4557.23947 & -5482.06984 & -6406.86818 \\
\hline
\end{tabular}

Table S16: Relative energy in $\mathrm{kcal} / \mathrm{mol}$ after optimization on TPSS-D3(BJ)/def2-TZVP level of different single filled hostguest structures, referenced to the single host and guest molecules.

\begin{tabular}{|c|c|c|c|c|c|}
\hline Guest & [9]CPP & [12]CPP & 1 & 2 & 3 \\
\hline benzene & -12.01396 & -9.46232 & -14.03463 & -12.09616 & -10.12659 \\
\hline methylbenzene & -13.95844 & -10.27803 & -14.89679 & -13.69255 & -11.90323 \\
\hline ethylbenzene & -15.15169 & -12.09507 & -17.26015 & -15.50423 & -11.95154 \\
\hline$i$-propylbenzene & -16.72679 & -13.21630 & -17.67852 & -16.81755 & -15.82783 \\
\hline$t$-butylbenzene & -19.57396 & -12.67970 & -20.04481 & - & -15.15479 \\
\hline 1,2-dimethylbenzene & -16.85470 & -12.94389 & -18.79352 & -15.71162 & -13.58398 \\
\hline 1,2-diethylbenzene & -20.32851 & -13.09884 & -20.83549 & -16.77164 & -14.55364 \\
\hline 1,2-di-i-propylbenzene & -22.34894 & -13.83361 & -19.44313 & -19.03565 & -18.43874 \\
\hline 1,3-dimethylbenzene & -15.55382 & -12.00918 & -17.77087 & -14.54576 & -14.96926 \\
\hline 1,3-diethylbenzene & -19.62026 & -14.70622 & -18.47577 & -18.66625 & -16.16573 \\
\hline 1,3-di-i-propylbenzene & -18.54232 & -16.35883 & -22.39177 & -17.96893 & -16.24124 \\
\hline 1,3-di-t-butylbenzene & -21.12379 & -14.90632 & -20.33224 & -23.83890 & -18.09547 \\
\hline 1,4-dimethylbenzene & -15.70096 & -11.69881 & -17.00932 & -15.13655 & -13.79914 \\
\hline 1,4-diethylbenzene & -20.60172 & -15.90311 & -19.50580 & -20.65084 & -15.36563 \\
\hline 1,4-di-i-propylbenzene & -17.77965 & -15.40758 & -20.66922 & -22.18948 & -16.82295 \\
\hline 1,4-di-t-butylbenzene & -18.60982 & -16.55702 & -22.49517 & -22.92547 & -18.66235 \\
\hline 1,2,3-trimethylbenzene & -17.66435 & -13.07260 & -17.74119 & -16.11865 & -14.54930 \\
\hline 1,3,5-trimethylbenzene & -16.42268 & -13.27346 & -18.44445 & -15.58578 & -15.87708 \\
\hline 1,3,5-triethylbenzene & -22.62507 & -17.14565 & -23.67784 & -19.91824 & -20.17300 \\
\hline 1,3,5-tri-i-propylbenzene & -21.97723 & -17.06054 & -23.99815 & -20.88428 & -19.27394 \\
\hline 1,3,5-tri-t-butylbenzene & -20.89035 & -17.60036 & -18.82082 & -22.57137 & -20.06340 \\
\hline 1,2,3,5-tetramethylbenzene & -18.76176 & -14.26372 & -21.13742 & -17.08486 & -14.10424 \\
\hline 1,2,3,4,5-pentamethylbenzene & -19.61505 & -13.88223 & -21.57880 & -18.58838 & -17.15454 \\
\hline $\begin{array}{l}\text { 1,2,3,4,5,6-hexamethylbenzene } \\
\text { 1,2,3-triethyl-4,5,6- }\end{array}$ & -21.14842 & -14.75015 & -22.37765 & -20.33992 & -19.89526 \\
\hline $\begin{array}{l}\text { trimethylbenzene } \\
135 \text {-triethyl-2 } 46 \text { - }\end{array}$ & -22.19942 & -18.22530 & -19.74838 & -22.11807 & -17.82005 \\
\hline $\begin{array}{l}\text { trimethylbenzene } \\
\text { 1,3,5-tri-i-propyl-2,4,6- }\end{array}$ & -21.39739 & -17.12049 & -24.53577 & -23.68296 & -20.07578 \\
\hline $\begin{array}{l}\text { trimethylbenzene } \\
\text { 1,3,5-triethyl-2,4,6-tri-j- }\end{array}$ & -24.90597 & -18.58675 & -23.18933 & -24.58533 & -22.21933 \\
\hline propylbenzene & -28.99826 & -20.05792 & -25.50219 & -32.53915 & -24.56004 \\
\hline
\end{tabular}


To evaluate the impact of the analyte's substitution pattern, i.e. in the 1,2-, 1,3-, -or 1,4position, we analyzed the interaction energies of the corresponding analyte/host assemblies by computational methods. We find that the calculated interaction energies are decreasing with increasing macrocycle size for both, the [n]CPP and the lemniscate series due to the increasingly incomplete filling of the macrocyclic cavity. This trend is opposite to the observed affinity in the QCM measurements which is strongly suggesting a complex interplay of porosity analyte/ as well as analyte/analyte interactions energies for the final affinity.

Table S17: Relative energy in $\mathrm{kcal} / \mathrm{mol}$ after optimization on TPSS-D3(BJ)/def2-TZVP level of different single filled hostguest structures, referenced to the single host and guest molecules, for the dimethyl- and diethyl-series.

\begin{tabular}{lcccccc} 
Guest & SASA / $\AA^{2}$ & [9]CPP & [12]CPP & $\mathbf{1}$ & $\mathbf{2}$ & $\mathbf{3}$ \\
\hline 1,2-dimethylbenzene & 277.4 & -16.85 & -12.94 & -18.79 & -15.71 & -13.58 \\
1,3-dimethylbenzene & 283.5 & -15.55 & -12.01 & -17.77 & -14.55 & -14.97 \\
1,4-dimethylbenzene & 283.7 & -15.70 & -11.70 & -17.01 & -15.14 & -13.80 \\
1,2-diethylbenzene & 325.3 & -20.33 & -13.10 & -20.84 & -16.77 & -14.55 \\
1,3-diethylbenzene & 335.5 & -19.62 & -14.71 & -18.48 & -18.67 & -16.17 \\
1,4-diethylbenzene & 334.0 & -20.60 & -15.90 & -19.51 & -20.65 & -15.37 \\
\hline
\end{tabular}

The preferred docking position of the analyte in the distorted macrocycles of the lemniscate series were studied by computational methods. To this end, adsorption geometries of a single $p$-xylene molecule in host 2 were generated manually as a model system. The interaction energies were analyzed at TPSS-D3(BJ)/def2-TZVP//TPSS-D3(BJ)/def2-TZVP level. A selection of the energetically most favorable adsorption geometries is depicted in Fig. S53. Our results indicate, that the preferred binding sides in this test system are in macrocyclic cavity due to higher contact surface of the analyte with the polyphenylene backbone of lemniscate $\mathbf{2}$ as compared to adsorption at the $\mathrm{V}$-shaped binding pocket of the spirobifluorene unit outside the macrocycles (and also at the polyparaphenylene backbone outside the macrocycle, not shown). In the bulk material, however, our computations suggest that the V-shaped binding sides in the interstitial space can become energetically comparable depending on the supramolecular arrangement of the lemniscate as well as the analyte used. Here, we would like to stress the fact, that within the macrocycle the energetic difference between the various adsorption geometries at the $\mathrm{V}$-shaped

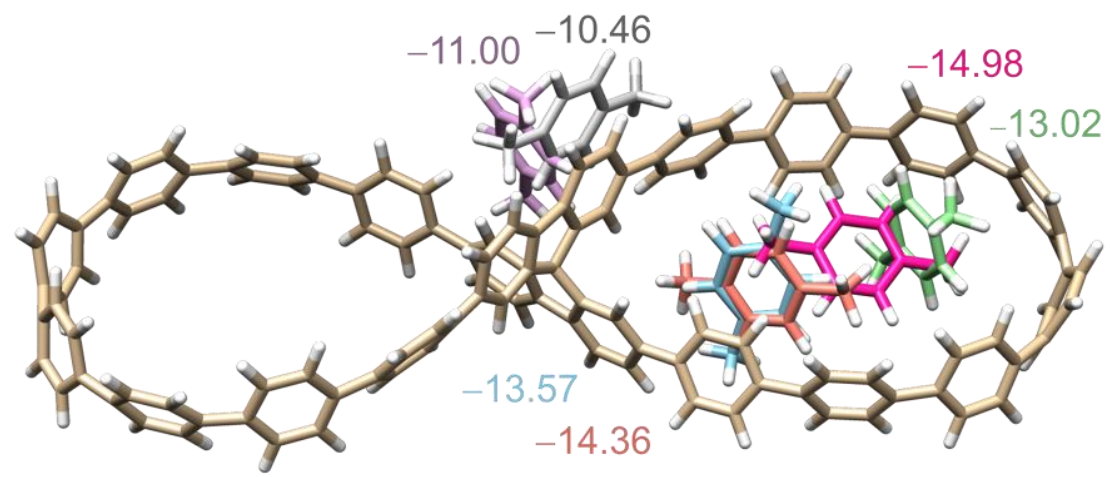

Figure S53: Optimized geometry of different docking positions and stabilization energies of $p$-xylene in compound 2 on TPSS-D3(BJ)/def2-TZVP level. 
spirobifluorene end and the curved polyparaphenylene end is negligible and thus the particular geometric environment of the central spiro-motif is not expected to significantly contribute to the generally higher affinities of the lemniscates as compared to the [n]CPP series found in the QCM study.

\section{Dipole Moments}

Dipole moments of the examined hosts or partial structure motives of it, were calculated with TURBOMOLE 7.2 on TPSS-D3(BJ)/def2-TZVP//TPSS-D3(BJ)/def2-TZVP level. To get an estimate about the polarity within the cavities of the lemniscates, dipole moments were calculated for one individual loop of all-hyrocarbon compound 1 and bipyridyl-containing lemniscate 4 , respectively (Fig. S54). The dipole moment of one half of 4 is 4.42 Debye and of 11.22 Debye. Both dipole moments point in the same direction towards the spiro-center (according to the convention that the dipole moment points from negative to positive charge).
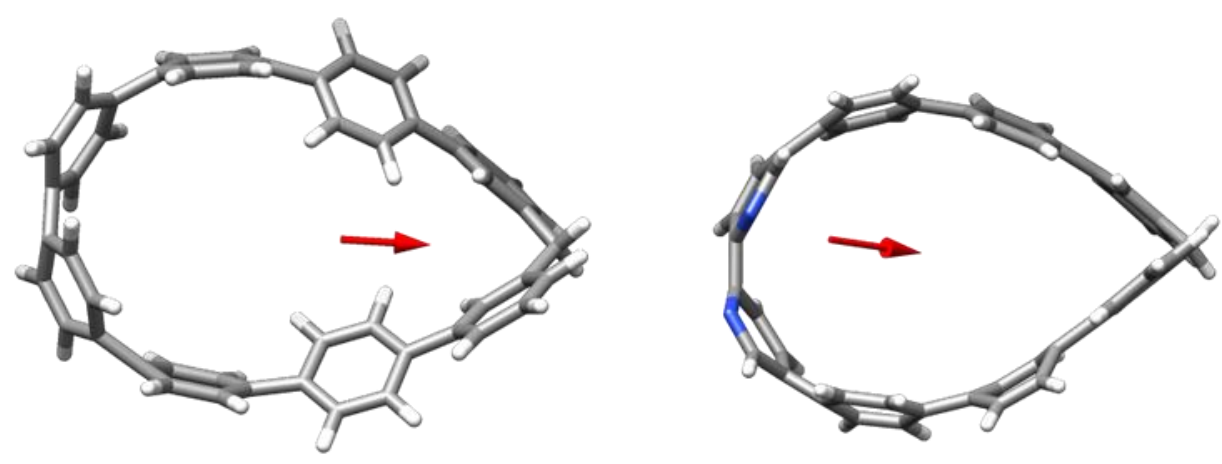

Figure S54: One half of 1 (left) and 4 (right) with visualization of the direction of the dipole moment, calculated on TPSS$D 3(B J) / d e f 2-T Z V P$ level of theory. Note that the arrow's length doesn't represent the absolute value of the dipole moment.

\section{Bulk Analyte/Host Model Systems}

Four different guests ( $p$-xylene, toluene, benzene, 1,3,5-triethylbenzene) were docked into host crystal ([9]CPP, [12]CPP, compounds 1-3, and quinquephenyl) excerpts of four unit cells up to a number of 50 guest molecules, applying the intermolecular force field $x T B-I F F^{[33]}$ as implemented in the $x t b 6.1^{[31]}$ program package. The resulting structures remained unoptimized, due to the system's size. The number of guest molecules fitting into the crystal was determined manually by counting. The crystal structures were received from the experimental X-ray diffraction data. Working tools for creating and visualizing molecules were molden, ${ }^{[34]}$ Avogadro, ${ }^{[35]}$ and mercury. ${ }^{[36]}$ 


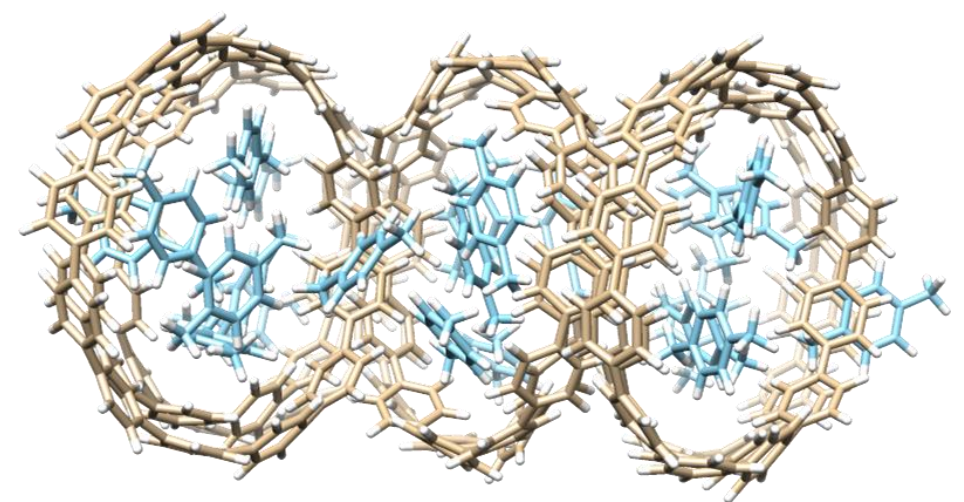

Figure S55: $p$-Xylene in partially filled host crystal excerpt of [12]CPP as demonstration for the examined dimensions.

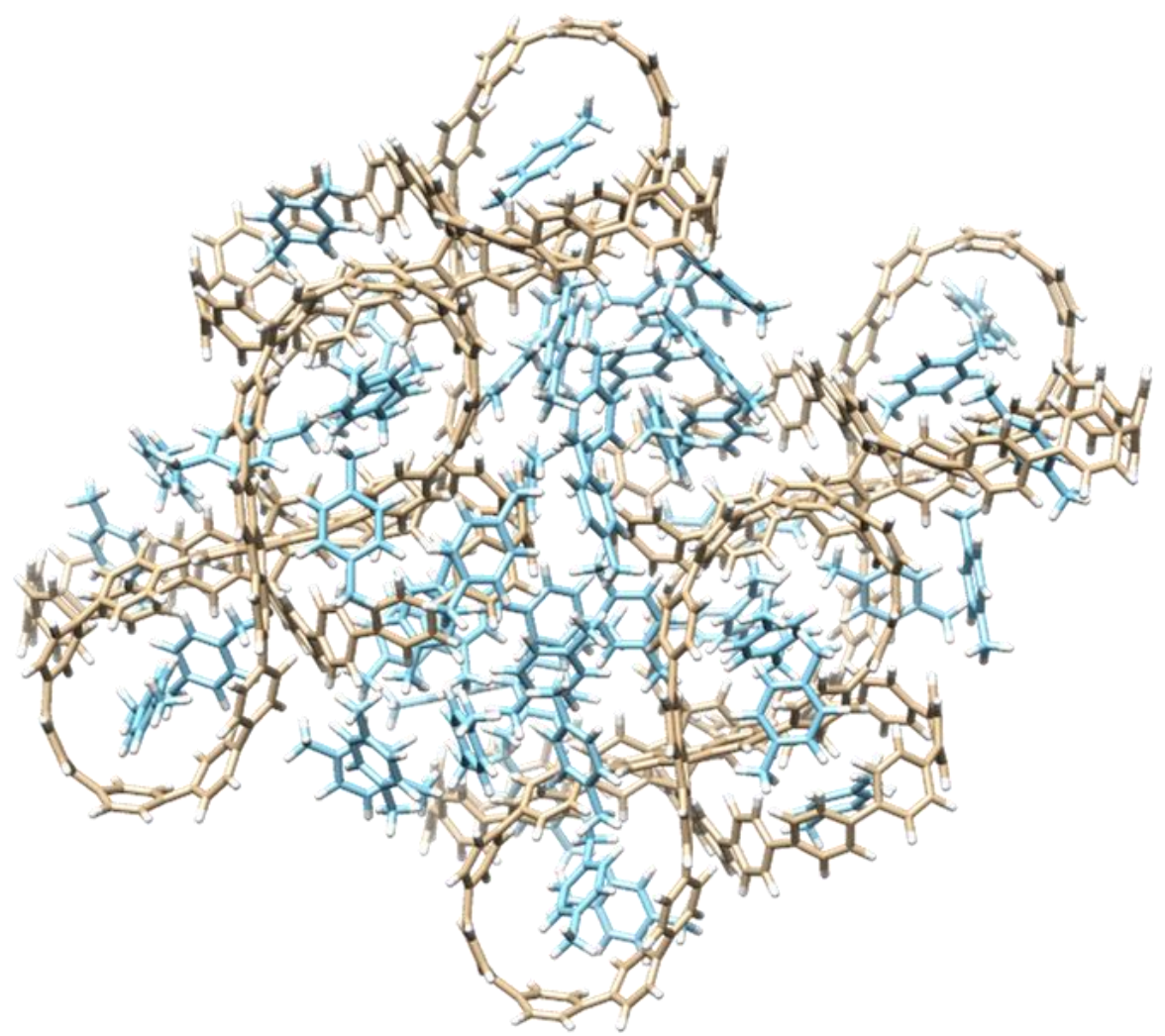

Figure S56: $p$-Xylene in partially filled host crystal excerpt of compound 1 as demonstration for the examined dimensions. 


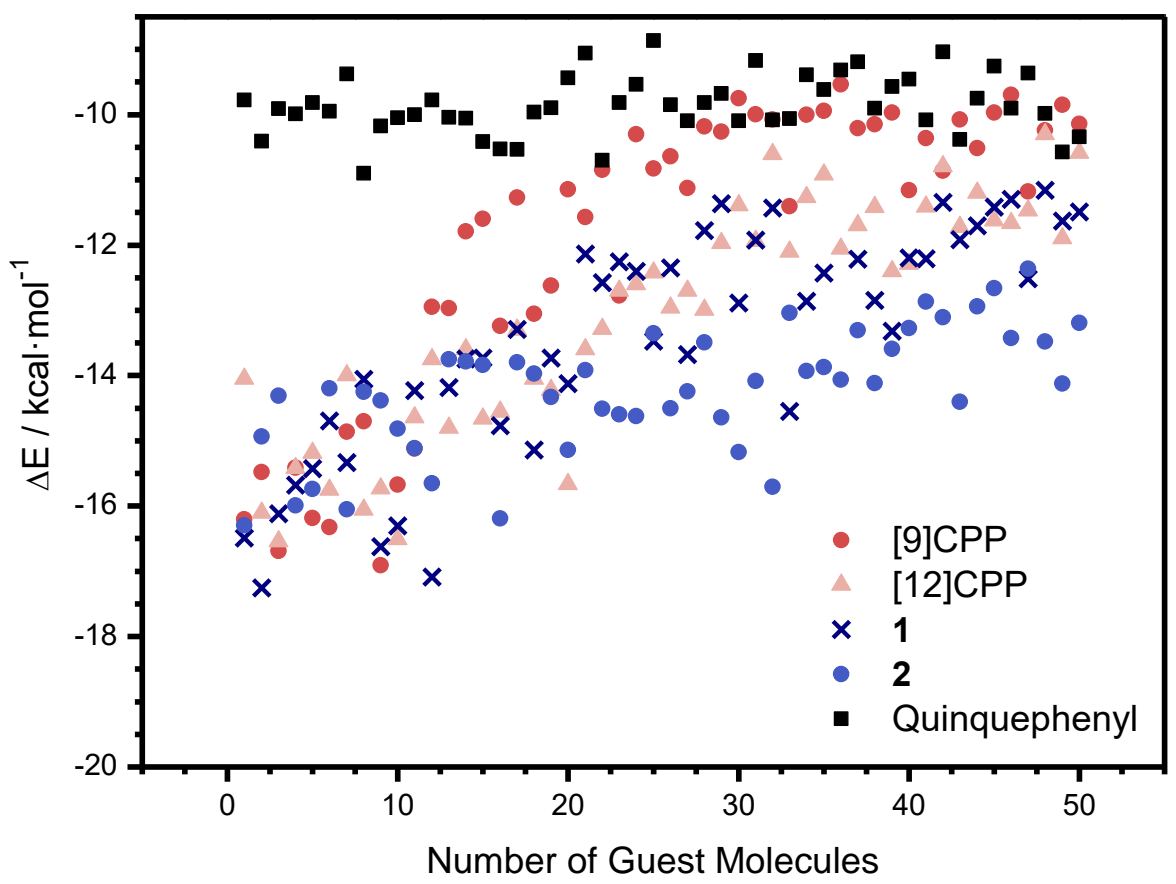

Figure S57: Relative energy in kcal of single point calculations with $x T B-I F F$ for stepwise docking of benzene into different host-crystals, referenced to the previous docked structure and a new guest.

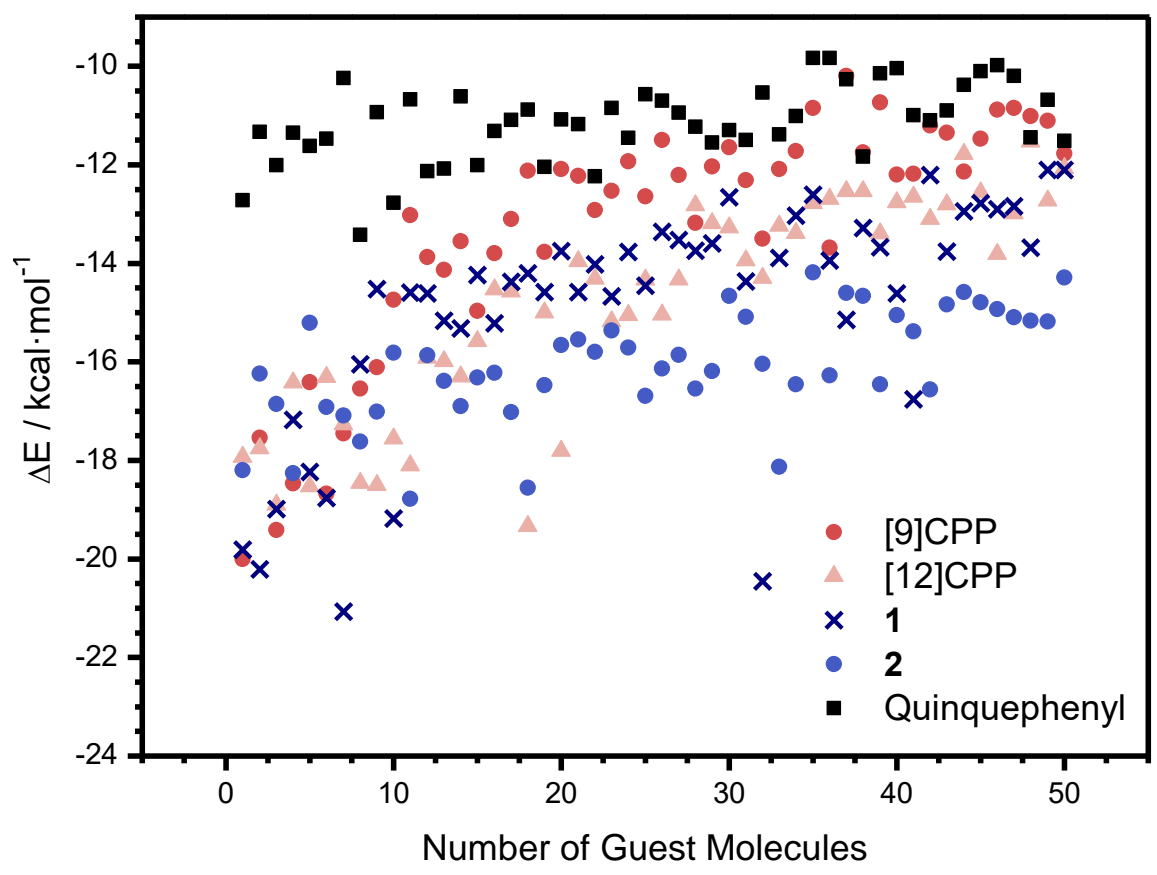

Figure S58: Relative energy in kcal of single point calculations with $x T B$-IFFfor stepwise docking of toluene into different host-crystals, referenced to the previous docked structure and a new guest. 


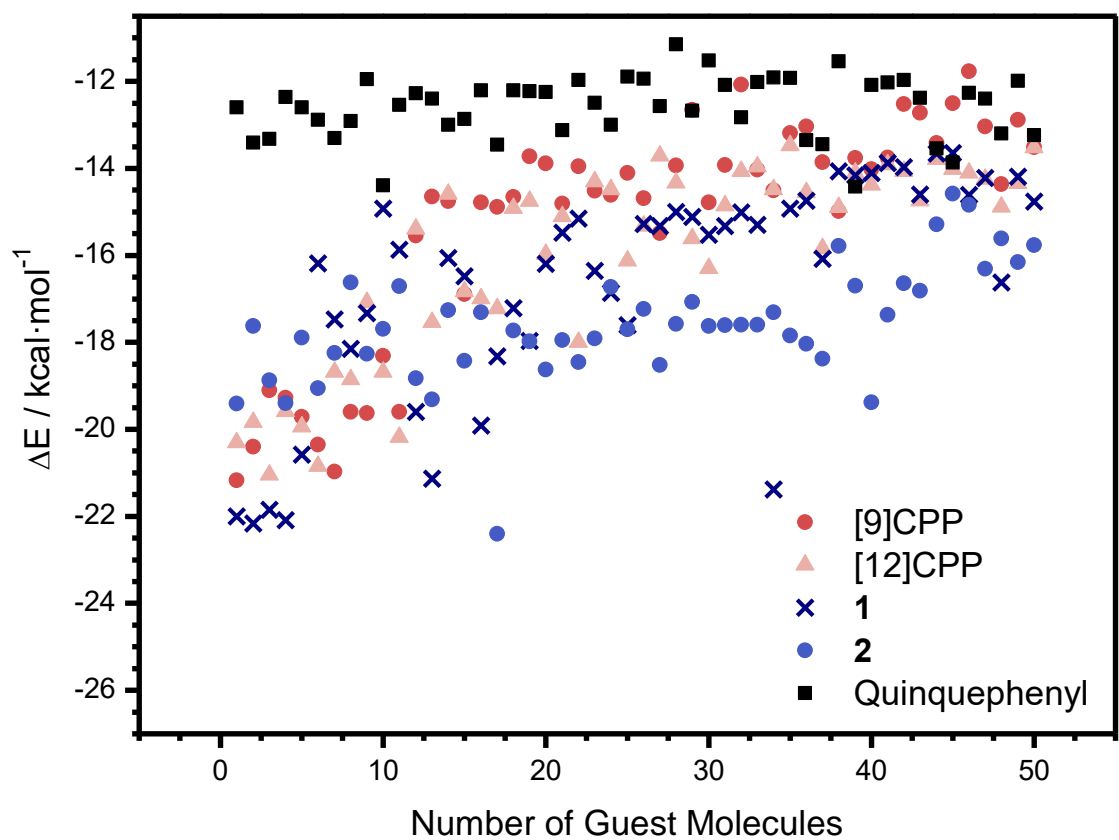

Figure S59: Relative energy in kcal of single point calculations with $x T B$-IFF for stepwise docking of $p$-xylene into different host-crystals, referenced to the previous docked structure and a new guest.

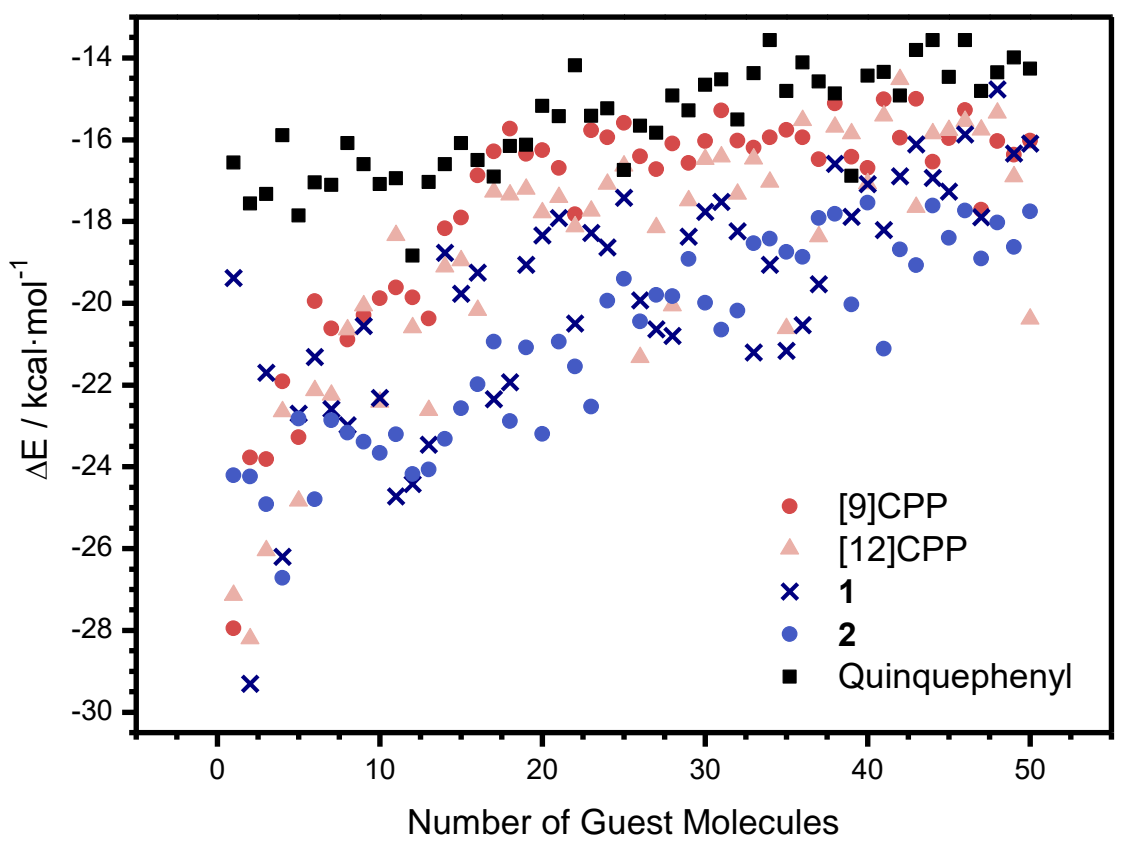

Figure S60: Relative energy in kcal of single point calculations with $x T B$-IFF for stepwise docking of $1,3,5$ triethylbenzene into different host-crystals, referenced to the previous docked structure and a new guest. 


\section{References}

[1] V. K. Patel, E. Kayahara, S. Yamago, Chem. - Eur. J. 2015, 21, 5742.

[2] E. P. Jackson, T. Sisto, E. R. Darzi, R. Jasti, Tetrahedron 2016, 72, 3754.

[3] A. B. Pangborn, M. A. Giardello, R. H. Grubbs, R. K. Rosen, F. J. Timmers, Organometallics 1996, 15, 1518.

[4] G. M. Sheldrick, Bruker/Siemens Area Detector Absorption Correction Program, Bruker AXS, Madison, WI, 1998.

[5] P. van der Sluis, A. L. Spek, Acta Crystallogr. A Found. Crystallogr. 1990, 46, 194.

[6] G. M. Sheldrick, Acta Crystallogr. C Struct. Chem. 2015, 71, 3.

[7] J. Rouquerol, P. Llewellyn, F. Rouquerol in Studies in Surface Science and Catalysis, Vol. 160 (Ed.: P. L. Llewellyn), Elsevier, Amsterdam, 2007, pp. 49-56.

[8] I. Pyka, D. Ryvlin, S. R. Waldvogel, ChemPlusChem 2016, 81, 926.

[9] M. Brutschy, D. Lubczyk, K. Müllen, S. R. Waldvogel, Anal. Chem. 2013, 85, 10526.

[10] A. Mujahid, A. Afzal, F. L. Dickert, Sensors 2019, 19, 4395.

[11] A. Wessels, B. Klöckner, C. Siering, S. R. Waldvogel, Sensors 2013, 13, 12012.

[12] G. Sauerbrey, Z. Physik 1959, 155, 206.

[13] A. K. Yatsimirsky, V. M. Mirsky (Eds.) Artificial receptors for chemical sensors, Wiley-VCH, Weinheim, Germany, 2011.

[14] a) B. Neubig, W. Briese, Das große Quarzkochbuch. Quarze, Quarzoszillatoren, Quarz- und Oberflächenwellenfilter (SAW), Meßtechnik, Franzis, Feldkirchen, 1997; b) A. Janshoff, C. Steinem (Eds.) Springer series on chemical sensors and biosensors, Vol. 5, Springer Berlin Heidelberg, Berlin, Heidelberg, 2007.

[15] a) C. Heil, G.R. Windscheif, S. Braschohs, J. Flörke, J. Gläser, M. Lopez, J. Müller-Albrecht, U. Schramm, J. Bargon, F. Vögtle, Sens. Actuators B Chem. 1999, 61, 51; b) J. B. Fenn, Angew. Chem. Int. Ed. 2003, 42, 3871; C) J. B. Fenn, Angew. Chem. 2003, 115, 3999.

[16] D. Ryvlin, O. Dumele, A. Linke, D. Fankhauser, W. B. Schweizer, F. Diederich, S. R. Waldvogel, ChemPlusChem 2017, 82, 493.

[17] Jobin Yvon Horiba, "A Guide to Recording Fluorescence Quantum Yields.".

[18] W. M. Haynes, D. R. Lide, T. J. Bruno, CRC handbook of chemistry and physics. A ready-reference book of chemical and physical data, CRC Press, 2013.

[19] a) S. Grimme, J. Antony, S. Ehrlich, H. Krieg, J. Chem. Phys. 2010, 132, 154104; b) E. R. Johnson, A. D. Becke, J. Chem. Phys. 2005, 123, 24101; c) A. D. Becke, E. R. Johnson, J. Chem. Phys. 2005, 123, 154101; d) J. Tao, J. P. Perdew, V. N. Staroverov, G. E. Scuseria, Phys. Rev. Lett. 2003, 91, 146401.

[20] F. Weigend, R. Ahlrichs, Phys. Chem. Chem. Phys. 2005, 7, 3297.

[21] E. Caldeweyher, S. Ehlert, A. Hansen, H. Neugebauer, S. Spicher, C. Bannwarth, S. Grimme, J. Chem. Phys. 2019, 150, 154122.

[22] K. Eichkorn, O. Treutler, H. Öhm, M. Häser, R. Ahlrichs, Chem. Phys. Lett. 1995, 242, 652.

[23] a) R. Ahlrichs, M. K. Armbruster, R. A. Bachorz, M. Bär, H. Baron, R. Bauernschmitt, F. A. Bischoff, S. Böcker, A. M. Burow, N. Crawford, P. Deglmann, F. D. Sala, M. Diedenhofen, M. Ehrig, K. Eichkorn, S. Elliott, D. Friese, F. Furche, A. Glöß, N. Graf, L. Grajciar, F. Haase, M. Häser, C. Hättig, A. Hellweg, B. Helmich, S. Höfener, H. Horn, C. Huber, U. Huniar, M. Kattannek, W. Klopper, A. Köhn, C. Kölmel, M. Kollwitz, M. Kühn, K. May, P. Nava, C. Ochsenfeld, H. Öhm, M. Pabst, H. Patzelt, D. Rappoport, O. Rubner, A. Schäfer, G. Schmitz, U. Schneider, M. Sierka, D. P. Tew, O. Treutler, B. Unterreiner, M. von Arnim, F. Weigend, P. Weis, H. Weiss, and N. Winter, TURBOMOLE 7.2 2017, Universität Karlsruhe. See also: http://www.turbomole.com.; b) F. Furche, R. Ahlrichs, C. Hättig, W. Klopper, M. Sierka, F. Weigend, WIREs Comput. Mol. Sci. 2014, 4, 91.

[24] Y. Segawa, H. Omachi, K. Itami, Org. Lett. 2010, 12, 2262.

[25] Gaussian 09, Revision A.02, M. J. Frisch, G. W. Trucks, H. B. Schlegel, G. E. Scuseria, M. A. Robb, J. R. Cheeseman, G. Scalmani, V. Barone, G. A. Petersson, et. al., Gaussian, Inc., Wallington CT 2009.

[26] a) P. J. Stephens, F. J. Devlin, C. F. Chabalowski, M. J. Frisch, J. Phys. Chem. 1994, 98, 11623; b) Lee, Yang, Parr, Phys. Rev., B Condens. Matter 1988, 37, 785.

[27] a) A. Schäfer, H. Horn, R. Ahlrichs, J. Chem. Phys. 1992, 97, 2571; b) F. Weigend, Phys. Chem. Chem. Phys. 2006, 8, 1057.

[28] R. E. Stratmann, G. E. Scuseria, M. J. Frisch, J. Chem. Phys. 1998, 109, 8218.

[29] a) R. Bauernschmitt, R. Ahlrichs, Chem. Phys. Lett. 1996, 256, 454; b) Carole Van Caillie, Roger D. Amos, Chem. Phys. Lett. 1999, 308, 249; c) Carole Van Caillie, Roger D. Amos, Chem. Phys. Lett. 2000, 317, 159; d) M. E. Casida, C. Jamorski, K. C. Casida, D. R. Salahub, J. Chem. Phys. 1998, 108, 4439; e) F. Furche, R. 
Ahlrichs, J. Chem. Phys. 2002, 117, 7433; f) G. Scalmani, M. J. Frisch, B. Mennucci, J. Tomasi, R. Cammi, V. Barone, J. Chem. Phys. 2006, 124, 94107.

[30] a) K. Matsui, Y. Segawa, K. Itami, Org. Lett. 2012, 14, 1888; b) J. M. van Raden, S. Louie, L. N. Zakharov, R. Jasti, J. Am. Chem. Soc. 2017, 139, 2936.

[31] x. 6.1. S. Grimme, Mulliken Center for Theoretical Chemistry, University of Bonn, 2019, available upon request via e-mail to xtb@thch.uni-bonn.de.

[32] E. F. Pettersen, T. D. Goddard, C. C. Huang, G. S. Couch, D. M. Greenblatt, E. C. Meng, T. E. Ferrin, J. Comput. Chem. 2004, 25, 1605.

[33] S. Grimme, C. Bannwarth, E. Caldeweyher, J. Pisarek, A. Hansen, J. Chem. Phys. 2017, 147, 161708.

[34] G. Schaftenaar, J. H. Noordik, J. Comput. Aided Mol. Des. 2000, 14, 123-134. See also: http://cheminf.cmbi.ru.nl/molden, Molden V.5.7.

[35] M. D. Hanwell, D. E. Curtis, D. C. Lonie, T. Vandermeersch, E. Zurek, G. R. Hutchison, J. Cheminformatics 2012, 4, 17. See also: http://avogadro.cc/, Avogadro V.1.2.

[36] C. F. Macrae, I. J. Bruno, J. A. Chisholm, P. R. Edgington, P. McCabe, E. Pidcock, L. Rodriguez-Monge, R. Taylor, J. van de Streek, P. A. Wood, J. Appl. Crystallogr. 2008, 41, 466-470. See also: www.ccdc.cam.ac.uk, Mercury V.3.8. 